\title{
In-silico Exploration of Mouse Brain Dynamics by Stimulation explains Functional Networks and Sensory Processing
}

Andreas Spiegler ${ }^{\mathrm{a}}$, Javad Karimi Abadchi ${ }^{\mathrm{b}}$, Majid Mohajerani ${ }^{\mathrm{b}}$, and Viktor K. Jirsa ${ }^{\mathrm{c}}$

\author{
${ }^{a}$ Department of Neurology, Charité University Medicine Berlin, Germany \&
}

${ }^{\mathrm{b}}$ Canadian Center for Bahavioural Neuroscience, University of Lethbridge, 4401 University

7 Drive Lethbridge, Alberta T1K 3M4, Canada. Tel.: (403) 394-3950; Fax: 4033292775.

c Institut de Neurosciences des Systèmes, UMR Inserm 1106, Aix-Marseille Université, Faculté de Médecine, 27, Boulevard Jean Moulin, 13005 Marseille, France

\begin{abstract}
Sensory and direct stimulation of the brain probes its functional repertoire and the information

11 processing capacity of networks. However, a systematic exploration can only be performed in

12 silico. Stimulation takes the system out of its attractor states and samples the environment of 13 the flow to gain insight into the stability and multiplicity of trajectories. It is the only means 14 of obtaining a complete understanding of the healthy brain network's dynamic properties. We 15 built a whole mouse brain model with connectivity derived from tracer studies. We 16 systematically varied the stimulation location, the ratio of long- to short-range interactions, 17 and the range of short connections. Functional networks appeared in the spatial motifs of 18 simulated brain activity. Several motifs included the default mode network, suggesting a 19 junction of functional networks. The model explains processing in sensory systems and 20 replicates the in vivo dynamics after stimulation without parameter tuning, emphasizing the
\end{abstract} 21 role of connectivity.

22 Keywords: Large-scale network, brain modeling, stimulation, mouse brain, brain model, 23 sensory stimuli, voltage sensitive dye, brain dynamics, connectivity, resting state 


\section{INTRODUCTION}

Although many structural and functional components of brain networks are known across spatial and temporal scales, there is yet an integration to be performed enabling us to link brain activity to function in cognition, perception and action. The debate on representation of behavior is old and on-going (see for recent discussions, Huys et al., 2014; Krakauer et al., 2017; Pillai \& Jirsa, 2017), thereby converging towards acknowledging the dynamic nature of behavior, typically manifest in its capturing as mathematical objects suited to represent dynamics (Structured Flows on Manifold (SFM, see Pillai \& Jirsa, 2017), Dynamical Causal Modeling (DCM, Friston et al 2003), Heteroclinic Cycles (Laurent \& Rabinovich, 2008). This is less evident for brain dynamics, in which the field remains dominated by static representations of brain activity such as brain states (sleep, awake, seizure, behavior (sniffing, etc.)), prone to neo-phrenological interpretations and localizationist perspectives on the representation of function in brain activations (see, for instance, Dehaene et al., 2015; Calvert et al., 2004 and the discussion therein). To go beyond the sampling of brain states and localization of their activity focus, brain dynamics outside its attractor states needs to be systematically measured and evaluated from a dynamics perspective. Attempts in this domain have been made by groups quantifying resting and task dynamics through sequences of brain activation patterns (micro states: Van de Ville et al., 2010; Michel and Koenig, 2018), fit lowdimensional dynamic systems to event-related potentials (for instance in generative Bayesian models via DCM: David et al., 2006) and estimators of various forms of causality. Such model inversion relies in all cases on the data features selected for the inversion and model identifiability remains a principle source of uncertainty (Raue et al., 2009). In-silico brain modeling avoids these issues around model inversion and allows creating brain network dynamics under the constraints of biologically realistic connectivity. When the modeled brain source activity is placed in an appropriate neuroinformatics framework (see for instance http://www.thevirtualbrain.org), then biophysical forward solutions can be computed linking the source signals to functional neuroimaging signals as observed in real world situations. Insilico modeling of stimulated brain activity thus offers an excellent means of exploring brain dynamics inside and outside of attractor states, with a direct link to measurable brain signals.

Brain imaging techniques using electrodes (Buzsáki et al., 2012), two-photon microscopy (Denk et al., 1994; Svoboda \& Yasuda, 2006), and voltage-sensitive dye (VSD) imaging (Mohajerani et al., 2013) provide little (but thoroughly precious) direct insight into brain dynamics on the large scale of an individual brain because of the restriction on recordings (e.g., limited number of intraoperative electrodes and limited access to dyeable brain tissue in 
a single individual). Because the access is limited both to (i) the interventions on the living tissue, and to (ii) the measurements of its activity, systematic in-vivo explorations of brain dynamics are difficult to accomplish, especially not feasible in individuals within a given period of time. Brain models however allow performing experiments in silico extensively and systematically. Protocols in such 'virtual' experiments can mimic and thus accompany those used in-vivo, for instance, for validation purposes (e.g., behavior tasks, brain stimulation as well as resting state protocols). In addition to that, in-silico experiments allow for manipulations that are lethal in vivo and simply not practical in individual (e.g., removing brain tissue, cutting connections, changing physiology). However, in-silico experiments have the potential to give insights if and only if experimental data is amply incorporated in the modeling (models are biologically informed) and missing information is discussed and tested in terms of assumptions. To perform systematic exploration of the brain dynamics via stimulation in-silico we exploited the Allen Brain Atlas (ABA), which compiles tracer studies from thousands of mice to construct an anatomical model of an average mouse brain, see Figure 1. Such a model is biologically informed concerning its geometry and the connectivity of its parts the brain areas. The structure of the brain constrains the brain function and dysfunctions (and vice versa) that are reflected in brain signals (measured, for example, by electrophysiology, VSD imaging, functional MRI). Our modeling approach of the brain assumes that (i) the brain structure given by the ABA is stable within a period of the measurements (e.g., for one hour), and that (ii) local dynamics at each node of the brain network (forming spatially extended brain areas in the model) provides the brain with activity. The assumption about the brain anatomy is realistic because the structure (i.e., geometry and connectivity) is experimentally derived and concerns the large scale of a brain. The assumption about the dynamics is less grounded because less sufficient evidence is available to infer the local dynamics from measurements. For instance, by using electrophysiology local activity (in a distinct brain structure) can be recorded. However, recordings from a limited number of sites do provide information about characteristic changes in space and time but do not clarify if an activation pattern related to function or dysfunction (e.g., epileptic discharges) originated locally or emerged throughout the actions in the network. Brain stimulation techniques are here an appropriate method to infer local characteristics (e.g., cortical excitability) by probing the formation of characteristic patterns in the brain activity (e.g., spreading and dissipation of activity from the stimulation site). The scope of this study is to probe the mouse brain structure by stimulation. For this purpose we assume that the dynamics at each network node is identical. This assumption is rather 
idealizing but it allows for equally probing the influence of connectivity and each of its parts by stimulation. That means that differences in the organization of brain activity in the model do not originate from the differences in the local dynamics but solely originate from the largescale brain network of the average mouse brain derived from the experimental data provided by the ABA. In this sense, we have provided each network node with simple dynamics that allows it to respond to stimulation and incoming input from other brain regions with a damped oscillation in the gamma range (see Figure 2). Rhythmic activity at each node is assumed to allow the transmission times of the connections between brain areas to be effective. For this purpose the rhythms need to be fast (e.g., faster than $40 \mathrm{~Hz}$ ) and the conduction speed needs to be slow (e.g., less than $1 \mathrm{~m} / \mathrm{s}$ ) in consideration of the length of axonal fibers connecting brain areas (e.g., mean length of about $6.25 \mathrm{~mm}$ ). We assume a natural frequency of $42 \mathrm{~Hz}$ at each network node and $1 \mathrm{~m} / \mathrm{s}$ of conduction speed - both within biologically plausible ranges (see section 'Conduction speed and transmission delays' and section 'Local areas and their dynamics' in the Results). By this simple dynamics at each node we probe the experimentally derived large-scale brain network to produce functionally relevant organizations in the activity by stimulation. Dynamics of simple systems (e.g., planar systems can show smooth and rhythmic responses to stimulation) are often included in systems that are more complex (e.g., Jansen-Rit model within certain ranges for each parameter). However, complex behavior (e.g., occurrence of spike-wave complexes (Jirsa et al., 2014) or epileptic discharges in distant brain areas (Proix et al., 2014)) indicates the complexity of systems and therefore cannot be embedded in simple systems. This strategy provides a discussion about the integration and emergence of function and dysfunctions in the brain, especially at the large scale of entire brains (for a review see Deco et al., 2011; and Breakspear, 2017). If large-scale models are able to provide plausible brain dynamics than the large-scale network provides entry points for interventions, useful for diagnostics and therapy among others.

We have used focal stimulation to render the brain dynamics visible in the brain response. The stimulation is brief and, to be effective, defined by the characteristic time of the intrinsic dynamics (gamma activity). We systematically stimulated every single brain area and investigated the subsequently induced activity in the brain responses. We simulated the whole-brain mouse model on a computer using the large-scale brain network simulation engine The Virtual Brain (TVB). For exploring the mouse brain dynamics in silico, we systematically varied three model parameters, namely (i) the ratio to which extend brain areas can interact over short- and long-ranges, (ii) the range of short connections that are 
125 homogeneous on the isocortex, and (iii) the location of the focal stimulation. The extent to which information is processed over short- or long-range SC is unclear and focal stimulation is closely related to direct electrical, sensory and optical stimulation. We identified consistent spatial motifs of mouse brain activity after stimulation called dynamically responsive networks (DRNs) using dimension reduction methods. We found the experimentally known

130 functional networks back in the DRNs. Interestingly, the salience and the default mode network spanned several motifs indicating the salience and default mode network to be a junction for functional networks. To test whether the mouse brain activity can be exclusively inferred from the underlying topology, we performed graph theoretic analysis of the model structure. We furthermore investigated the organization of brain activity following sensory pathways. The model structure reflects the sensory systems, namely: the auditory pathways, the visual pathways, the whisker pathways and the pathways for the limbs. Finally, with the developed large-scale mouse brain network model we had, for the first time, the chance to systematically investigate peripheral pathways, especially, the processing of sensory data. We showed that the in-silico brain responses resemble in-vivo brain dynamics after stimulation.

\section{RESULTS}

140 The result section is divided into two parts. The first part contains the results of the in-silico

141 exploration study by means of focal stimulation. The second part contains the results of 142 compiling the whole mouse model. This modeling work is fundamental to the in-silico 143 exploration but it is also suitable for many more diverse studies, and in this sense, the 144 modeling work stands for itself. The modeling work of the mouse brain was thus not solely 145 driven by the goal of performing in-silico brain stimulation but is rather driven by the 146 objective of obtaining a systemic and holistic description of the brain. We have used brain 147 stimulation as an appropriate concept and the results emphasize the potential of dynamic 148 models to study the functional repertoire of the brain and the information processing capacity 149 of networks.

1501 In-silico exploration of mouse brain dynamics by means of stimulation

151 The model allows each brain areas to communicate through short- and long-range connections 152 with other nearby or distant areas. The local dynamics (at each brain area) distinguish both 153 types of structural connectivity in the mathematical description, where a model (order) 154 parameter $\alpha$ sets the ratio and the weight of short- and long-range connections. The parameter $155 \alpha$ simply scales all long- and all short-range connectivity weights (regarding absolute values) 
156 with no effect on the relative weights (between short-range connections or between longrange connections).

158 We consider this ratio of homogeneous SC to heterogeneous SC as a degree of freedom and performed a parametric study. The ratio has been estimated. For instance, Braitenberg and

160 Schüz (1998) assessed that pyramidal cells have synapses in equal shares from long-range and

161 local axons. However, the ratio of homogeneous SC to heterogeneous SC mainly depends on

162 the resolution of the used geometrical model of the cortex, and with that the representation of

163 the SC, and the local dynamic model (e.g., canonical model, neural mass model), which is able to incorporate local connectivity (for more detail, see Spiegler and Jirsa, 2013). At the extremes, (1) $\alpha=1$, that is, $0 \%$ of heterogeneous SC (thus, $100 \%$ of homogeneous SC gives two unconnected hemispheres where only nodes within the isocortices are locally and homogeneously connected) only allows activity to propagate locally from an isocortical stimulation site, and (2) $\alpha=0$, that is, $100 \%$ of heterogeneous SC (thus $0 \%$ of homogenous SC gives 512 purely heterogeneously connected brain areas with locally unconnected nodes) only allows activity to travel distances with time delays. The connectivity scaling factor $\alpha$ is varied as follows: $\alpha=\left\{\begin{array}{ll}0.0 & 0.20 .40 .60 .81 .0\}\end{array}\right.$.

172 Furthermore, since the spatial range of homogeneous SC is of $\mu \mathrm{m}$ up to $\mathrm{mm}$ (Braitenberg and 173 Schüz, 1998), we also consider it as a model parameter varying between $500 \mu \mathrm{m}$ and $1000 \mu \mathrm{m}$

174 (step size $100 \mu \mathrm{m}$ ). We then systematically stimulate each of the 512 areas with a large range 175 of connectivity parameter values (for the ratio and the spatial range), resulting in a total 176 number of 18,432 simulation trials.

177 The dynamically responsive networks (DRNs) are extracted by (i) determining the induced component of generated brain activity after stimulation (subtracting the evoked response at the stimulation site, that is, an isolated and unconnected node), (ii) then decomposing the time series (of the 14,400 neural masses) of stimulation-induced activity (in the time period between $250 \mathrm{~ms}$ and $750 \mathrm{~ms}$ after stimulation onset $t_{0}$ ) into principal components

182 (eigenvectors and corresponding eingenvalues) using the principal component analysis

183 (PCA), (iii) correlating the eigenspaces spanned by the components that cover 99\% of the 184 variance in the brain activity, (iv) clustering the eigenspaces, and (v) lumping the clusters after rotating the eigenspaces onto a common basis.

\section{Effect of structural connectivity with time delays on the brain response to stimulation}

187 Considering the scaling of structural connectivity at both extremes, that is, $0 \%$ of 188 heterogeneous SC and $100 \%$ of heterogeneous SC, the formation of dynamically responsive 189 networks (DRNs) due to stimulation is found to be different (see Figure 3). The DRNs of both 
190 extremes change in the communication via short-range and long-range connections. The results indicate that both extremes merge in the mix of the two connectivity types rather than reorganize (e.g., due to time delays in the long-range connections). One explanation for this behavior is that the short-range homogeneous connectivity pertains to a minority of 84 areas in the model whereas the long-range heterogeneous connectivity pertains simply all of the 512 areas. The DRNs are most similar when the 512 areas are only linked via the heterogeneous connectivity. The overall similarity decreases with scaling the homogeneous connectivity up (consequently the heterogeneous connectivity down) and clusters of similar DRNs emerge and become clearer (see Figure 3). The clusters due to different stimulation sites are however the same throughout. Within a cluster structures may appear for higher scaling of the homogeneous connectivity, but clusters do not move, split nor merge (as observed in a similar study for human; Spiegler et al., 2016). The behavior in the similarity of DRNs is unaffected by the spatial range of the local connections. Merely the similarities increase with increasing the range of local connections for higher proportions of the short-range connectivity (e.g., $80 \%$ ). All in all, both SC types of short- and long-range connections in the mouse model act as spatiotemporal filters of local dynamics in the emergent functional network. The results (Figure 3) do not support evidence for a reorganization of dynamics due to interplay between both types of SC in the mouse as compared to human models (Spiegler et al., 2016). Whereas the modeling work of the human brain mainly comprises cortical areas and their connectivities (i.e., local homogeneous short-range connections and connections via longrange white matter fiber tracts), more weight is given to subcortical areas in this mouse model. The findings indicate that for the mouse the homogeneous short-range SC in the isocortex is not sufficient for observing reorganizations of functional networks due to changes in the structural connectivities. Furthermore, the transmission time delays between areas are

214 found to be important in the emergence of the DRNs but less important in the filter action and 215 the interplay of the two connectivity types.

\section{Dynamically responsive networks (DRNs)}

217 The dynamically responsive networks (DRN) convey $99 \%$ of the stimulation-induced energy 218 in the brain composed by three principal components. By correlating the DRNs of different 219 stimulation sites and performing a permutation test, we identified twelve clusters that are consistent throughout the systematic parameter exploration. The 14,400 time series were used for extracting and correlating the DRNs. On this fine-grained level of 14,400 nodes, the isocortex is overrepresented (with 13,972 nodes), whereas on the coarse-grained level of the 512 brain areas the sub-isocortical areas (e.g., thalamus, cerebral nuclei) form the majority. 
To match the DRNs with the voltage-sensitive dye (VSD) imaging data, which mainly record brain activity from the isocortices, the clustering of the responsive network to stimulation of different sites was performed on the fine-grained level of the nodes.

The in-silico exploration provides a spatiotemporal map of consistent formation of a sequence of spatially similar brain activity and a map indicating which brain areas need to be stimulated (Figure 4, Panel A: the similarity matrix) to 'push' the brain in a particular state (see Figure 5 and Figure 4, Panels B, C: DRNs of the clusters 1 and 2 in Panel A). Stimulations specifically bias widespread functional networks. Spatial proximity of stimulation does not necessarily predict the similarity of induced activities. The same spatial organization of brain activity can be induced by stimulation of several sites scattered all over the brain.

\section{Statistics on DRNs}

We have performed statistics on the catalogue of DRNs (Figure 5) to (i) clarify whether the motifs are entangled in their individual parts, and to (ii) discuss how the formation of brain activity due to stimulation does follow the structure. For the first purpose we pair-wise correlated each of the three parts of each of the 24 motifs, that is, twelve motifs by stimulation of left brain areas and twelve motifs by stimulation of right areas (makes a total of 72 parts composing the 24 motifs). The significance level of $1 \%$ was Bonferroni corrected by the total number of components. In addition, three thresholds are applied to the correlation values $C$ to find strong $(C>0.8)$, good $(0.6<C \leq 0.8)$, and moderate agreements $(0.5<C \leq 0.6)$ among the 72 parts of the 24 motifs. Please note that for the rest of the manuscript we refer to the twelve motifs without indicating the stimulation side (left/right) because of the bilateral symmetry (in the spatial organization of brain activity due to left/right stimulation). The analysis reveals a total of five significantly distinct spatial patterns in the motifs of the DRNs, indicating their entanglements. The distinct spatial activation patterns could possibly be the structural links for the transfer of energy, that is, brain activity that may transmit information from one motif to another at rest over time. The reason for that conjecture is the occurrence of the five spatial patterns in the 12 motifs (see the highlighted parts in Figure 5), which, importantly, also allow an activation of more than one motif at a time (i.e., phrasing). The analysis also reveals six components that are rather unique (i.e., parts in motifs $2,4,6,7$, and 9 in panel $\mathbf{A}$ in Figure 5) and are not correlated to other motif parts. Considering the parts of the motifs and the occurrence of patterns, the motif with the number 7 (see, panel $\mathbf{A}$ in Figure 5 ) is the most unique one and the motif 1 is the most entangled one (followed by the entanglements in motifs 5,11, and 12) among all motifs. The pattern with the number 2 is 
motif 12. The most present pattern, that is, number 2 is also the pattern with the strongest correlations (out of a total of 11 parts, 7 parts are strongly correlated and 4 parts show good correlation), whereas pattern 1 shows only good correlations. Before interpreting the themes of the DRNs by relating the parts of the motifs (and thus the patterns) to experimentally known functional networks, we tested to which extent the topology in the structural connectivity predicts the brain activity due to stimulation.

The brain activity in response to stimulation follows the structural connections from the stimulation sites. Amongst the 13 graph theoretic measures on the structural long-range network (ABA), the direct projections from the site of stimulation best explain the brain response to stimulation though the correlation is rather moderate. The performance of the other twelve graph-theoretic measures (see Materials and Methods) is rather poor, that is, less correlated, weakly predictive and fewer significant configurations. The best prediction resulted from comparing the rank (Kendall's tau) of the activity at the network nodes after stimulation with the rank of connectivity strengths of the direct projection from the stimulation site. The rank correlation is weak but significant for most connectivity parameter configurations of the ratio $\alpha$ of long-range to short-range connections, and the spatial spread $\sigma$ of short-range connections. By utilizing this simple measure on the structural large-scale network, a high correlation value means that the energy induced by the stimulation spreads into areas of the brain that are directly connected to the stimulation site. We corrected the significance level of $5 \%$ (Bonferroni). The values of significant correlations are however fairly low with an average maximum value of $221.542 \times 10^{-3}$ across all parts of all motifs and all connectivity parameter configurations (maximum average correlation value of $253.889 \times$ $10^{-3}$ for all $1^{\text {st }}$ parts, $255.936 \times 10^{-3}$ for all $2^{\text {nd }}$ parts, and $338.724 \times 10^{-3}$ for all $3^{\text {rd }}$ parts across all motifs and all connectivity parameter configurations). The percentage of parameter configurations with significant rank correlation is: $48.981 \%$ across all parts and all motifs ( $49.706 \%$ for the $1^{\text {st }}, 56 \%$ for the $2^{\text {nd }}$, and $82.576 \%$ for the $3^{\text {rd }}$ parts). This low rate of significant configurations and the overall low correlation indicates that most of the activity induced by the stimulation spreads over the network on short and long ranges in a specific ways. The "direct network response" to stimulation seems to be captured by the $2^{\text {nd }}$ and $3^{\text {rd }}$ parts of the motifs because correlations are generally stronger for higher-order components, that is, $2^{\text {nd }}$ and $3^{\text {rd }}$ parts than the $1^{\text {st }}$ parts (and across the entire motifs). The first parts of each motif reflect the reorganization of the network activity caused by the stimulation. Because of the reorganization, these spatial formations of brain activity are however not necessarily traceable to the site, at which energy was introduced by stimulation. 
When considering stimulation-induced activities in the entire brain model (i.e., including all substructure), the maximum correlation is the lowest but the number of parameter configurations with significant correlations is the highest. This emphasizes the specificity of responses to substructures of the brain (e.g., thalamus, isocortex). For instance, the activity in the cerebellum shows the highest maximum correlation with the direct projections from the stimulation sites, whereas the number of configurations with significant correlation is fairly low. It is also interesting to mention that activity in the isocortical subplate shows no significant correlation with the underlying structure at all.

Comparison of dynamically responsive and functional networks

We compared the DRNs with distinct functional networks. The spatial organization of the nine experimentally known functional networks derived from Sforazzini et al., (2014) and Zingg et al., (2016) is shown in Figure 5, panel B for the isocortex. Four of the nine functional networks are correlated with the five patterns occurring in the parts of the motifs (see Figure 5). Interestingly, the sensory motor networks are not reflected in the motifs of the DRNs. Merely the network associated with the frontal eye field shows significant but moderate correlations with four parts of four motifs (see magenta pattern in Figure 5). Here, the lateral activity of the frontal eye field network (i.e., activity within one hemisphere) corresponds to pattern 4 (see Figure 5). The unique parts in motifs 2, 4, 6, 7, and 9 (see parts in Panel $\mathbf{A}$ that are not highlighted) are not correlated with one of the nine specific functional networks. Moreover, pattern 1 (highlighted in yellow) in motif 12 does not correspond to any of the nine functional networks. The default mode and the salience networks show similarities at the isocortical level. Both comprised most of the motifs (cyan pattern 2 in motifs 1 to 11 ).

314 Stimulation responses do activate to a lesser extent the lateral network (orange pattern 4 in 315 motifs 1 , and 3 to 5) and the medial network (gray pattern 3 in motifs 8 to 12), where the activity of both networks within one hemisphere (lateralized organization) is reflected. The default mode network (together with the salience network) is thus related in the model to the most flexible and sensitive pattern (cyan pattern number 2) that can activate and is involved in most of the motifs (1 to 11).

\section{Organization of brain activity following sensory pathways}

321 Stimulation of a brain area induces activity and traces out a spatiotemporal trajectory while 322 dissipating energy into specific parts of the network. The trajectory is characteristic and leaves a particular fingerprint of responses. With our large-scale mouse brain network model we have for the first time the chance to systematically investigate sensory pathways and the 
processing of sensory data. We focus on five systems, which are: the auditory pathways, the visual pathways, the whisker pathways and the pathways for the limbs. For each of the sensory system we extracted network descriptions according to the scientific consensus from the literature (see Materials and Methods). Figure 6 shows the networks of the sensory pathways. We then tested whether the experimentally derived large-scale brain network contains the textbook descriptions of each of these sensory pathway networks. The sensory networks (visual, auditory pathways and the pathways for the whisker, as well as fore and hind limb) are statistically well represented in the used ABA connectome. The sensory pathways in Figure 6 suggest that sensory processing follows specific paths of activations towards the primary somatosensory cortices. Activation of sensory processing can be ipsilateral to the sensory organ, such as for the whisker follicles up to the level of the midbrain (see Figure 6, panel D). The sensory processing usually crosses to the contralateral side of the sensory organ, indicating the integration of the left and right sensory organ of the same modality (see Figure 6), such as for the auditory pathways showing directed processing to the contralateral side (projections from the medulla to the pons) and then a processing mainly in the contralateral side through the midbrain and the thalamus towards the primary somatosensory areas in the isocortex (see Figure 6, panel B). Because our systematic exploration of brain dynamics via stimulation provides the brain responses to focal stimulation, we can investigate the brain responses and the DRNs to stimulation of areas involved in sensory processing. We can thus trace the activity and the dynamics in each of the sensory pathways. Note that such an investigation is possible in silico but hard to accomplish for an individual brain in vivo. The network model extracted from the ABA significantly contains the sensory pathways. The sensory pathways as shown in Figure 6 do reflect the primary paths of sensory processing and are thus a detail of a bigger picture, that is, the largescale brain network that spans the entire brain. The brain areas that are known to be involved in sensory processing may thus be connected to other brain areas and within the large-scale brain network and consequently involved in one or several functional networks. For this reason, it is evident that the entire brain dynamics needs to be considered in the sensory pathways. The brain areas in the sensory pathways as shown in Figure 6 provide guidance and prior knowledge for which the brain response to stimulation of exactly those areas and the occurring DRNs should be consistent and evolve. However, due to the large-scale network of

356 the brain and its organization, it is not trivial that the dynamics in the model are consistent across a sensory pathway of a specific modality (e.g., auditory system). Because the statistics on the DRNs, see section 'Statistics on DRNs' in the Results, indicate that the response are 
(although weak) best predicted by the strengths of the directly outgoing connections from the stimulation site, we analyzed the spatial spread of the energy in the brain responses (integral of the power over time, that is, $.2 \mathrm{~s}$ to $.8 \mathrm{~s}$ after stimulation). To test whether activity is focal or spread over the brain (or within a structure), we determined the brain areas that contain more than $95 \%$ of the total energy in a brain response (entire brain and within a brain structure). The results show that after stimulation of brain areas in the auditory pathways, the activity across all brain structures was most centered within the cerebellum in the flocculus and the copula pyramidis. In only one case (left and right stimulation of the nucleus of the brachium of the inferior colliculus of the midbrain) the activity was clearly centered in isocortex (laterointermediate area). The activity was mostly cumulated in the cerebellum. The distribution of activity was spreads and more complex in all other structures, namely olfactory areas, hippocampus, cortical subplate, cerebral nuclei, thalamus, hypothalamus, midbrain, pons, and medulla). In contrast to the auditory pathways, after stimulation of areas in the visual pathways the activity was most centered within the cerebellum but more moderate across stimulation sites. In only one case (left and right stimulation of the dorsal part of the lateral geniculate complex of the thalamus) the activity was clearly centered in isocortex around the laterointermediate area (better pronounced that for the case in the auditory pathway, that is, left and right stimulation of the nucleus of the brachium of the inferior colliculus of the midbrain). Activity after stimulation areas in the whisker pathways showed a clear centered distribution within the thalamus and within the cerebellum. The distribution of activity was more complex in all other structures. Furthermore, the results show that after stimulation of brain areas in the pathways of the limbs, the activity across all brain structures was less spread within the isocortex, the thalamus and the cerebellum. To clear whether activity spreads laterally we determined the brain areas that contain more than $95 \%$ of the total energy in a brain response in the entire brain and compared the location (left/right). The accumulation of activity is symmetric after stimulation of a given structure in the left and its complement in the right hemisphere. We found only weak asymmetries, which in turn point more towards a similar activation after left and after right stimulation. Most of the symmetric patterns are lateralized. Activity mostly cumulates either ipsilaterally or contralaterally to the stimulation site stimulation site (e.g., left or right LGN). However, non-lateralized symmetries are found in the medulla across all modalities. The pathways of the limbs show most non-lateralized symmetries across structures (olfactory areas, hippocampus, cerebral nuclei, pons, and medulla). Stimulation of areas in the auditory pathways indicates that most of the activity patters are lateralized and symmetric to left/right stimulation. The cerebral 
nucleus and the medulla show non-lateralized symmetries. Stimulation of areas in the visual pathways indicates that most of the activity patters are lateralized and symmetric to left/right stimulation. The medulla shows non-lateralized symmetries. Stimulation of areas in the pathways of the whiskers indicates that most of the activity patters are lateralized and symmetric to left/right stimulation. The pons and the medulla show non-lateralized symmetries. Stimulation of areas in the pathways of the limbs indicates that the activity patters are lateralized and symmetric to left/right stimulation. However, olfactory areas, hippocampus, cerebral nuclei, pons, and medulla show non-lateralized symmetries. To test whether the brain response to stimulation shows such symmetries as bilateral activations, we correlated the activity in the left areas with the activity of the corresponding areas on the right. A symmetric response means that the activity in the brain areas on the left side is similar to the ones on the right. An anti-symmetric response is indicated by a negative correlation and means that the activity on the left side is the reverse of that on the right. Not significant correlations (significance level of 5\%) do not support either type of symmetry. The results suggest dividing the activity in the isocortex from the rest. Consistently across all stimulation of all brain areas involved in the sensory pathways (across all modalities and left and right areas), the responses in the subisocortex are mostly symmetric, whereas isocortical patterns are more variable. Dependent on the stimulation the response in the isocortex shows no symmetry (sign for lateralization), anti-symmetry (left/right inverted activity) or symmetries (bilaterally symmetric). This is consistent for the auditory, visual, whisker and limb pathways. In addition to investigating the distribution of the response we can analyze the energy to determine the areas that are most active. The energy in the brain responses (integral of the power over time, that is, $.2 \mathrm{~s}$ to $.7 \mathrm{~s}$ after stimulation) indicates that the areas in the hypothalamus are the most active ones throughout stimulations, sites, and modalities. Their activation is lateralized and, in the 1st (dominant) part of a motif, contralateral to the stimulation sites in most of the cases (except vision, that is not lateralized). The most dominant areas in the hypothalamus are: the intermediate part of the periventricular hypothalamic nucleus, the lateral mammillary nucleus, and the arcuate hypothalamic nucleus. Ordering the areas to their strength of activity and then comparing between left structures and the complement on the right indicate a lateral symmetry. The brain areas that are most active after stimulation of areas across the auditory pathways are: the arcuate nucleus, the dorsal cochlear nucleus, the intermediate part of the periventricular nucleus, the abducens nucleus, the primary auditory area, and the laterointermediate area. The most active brain areas after 
and the intermediate part of the periventricular nucleus. Stimulation across the pathways of the whisker shows the following most active areas: the intermediate part of the periventricular nucleus, the primary somatosensory area of the mouth, the arcuate nucleus, the supplemental somatosensory area, and the ectorhinal area. The brain areas that are most active after stimulation of areas across the pathways of the limbs are: the intermediate part of the periventricular nucleus, the primary somatosensory area of the lower limb, the abducens nucleus, the gracile nucleus, the nucleus ambiguus, the laterointermediate area, the visceral area, and the lateral mammillary nucleus.

So far we have analyzed the brain response to stimulation of brain areas in sensory pathways independently. To clarify whether the brain responses are consistent for a certain sensory pathway summarized in Figure 6, for example, the auditory pathways from the cochlea to the primary auditory areas, we made use of the correlation of the responses and the clustering into characteristic motifs of the dynamically responsive networks (DRNs) as described in section 'Statistics on DRNs' in the Results. The catalogue of dynamically responsive networks and its stimulation sites (see Figure 4 and Figure 5) reveal whether the responses due to stimulation along the pathways belong to the same cluster. The catalogue shows that the responses are consistent and alter along the auditory pathways (bottom-up). The formation from the cochlea to the primary auditory areas can be subdivided into three motifs (from cochlea to cortex through medulla, pons, midbrain, and thalamus): (1) the motif with number 5 in Figure 5 due to stimulation in the medulla (dorsal and ventral cochlear nucleus), (2) the motif with number 9 in Figure 5 shared by stimulation of the nucleus of the trapezoid body of the medulla, the nucleus of the lateral lemniscus of the pons, and the nucleus of the brachium of the inferior colliculus of the midbrain, and (3) the motif with number 10 in Figure 5 shared by stimulation of the inferior colliculus (midbrain) and the medial geniculate complex of the thalamus. The catalogue shows that the responses are consistent throughout the visual pathways and have the same motif, that is, motif with number 10 in Figure 5. The catalogue shows that the responses are consistent and alter along the pathways of the whiskers (bottom-up). The formation from the whisker follicles to the barrel field of the primary somatosensory areas can be subdivided into three motifs (from follicles to cortex through medulla, pons, midbrain, and thalamus): (1) motif with number 6 in Figure 5 shared by caudal, interpolar, and oral part of the spinal nucleus of the trigeminal in the medulla as well as by the principal sensory nucleus of the trigeminal in the pons, (2) motif with number 6 in Figure 5 shared by stimulation in the midbrain (of the superior colliculus, sensory related) and in the thalamus (of the lateral dorsal nucleus), (3) the two thalamic areas, that is, the ventral posteromedial nucleus and the 
posterior complex of the thalamus share the same motif that has the number 8 in Figure 5 .

462 The response to stimulation of the nuclei of the medulla involved in the sensory pathways of 463 the limbs, that is, cuneate nucleus and gracile nucleus are correlated and have the same motif 464 (number 6 in Figure 5). The responses due to stimulation of the nuclei in the thalamus, that is, 465 the ventral posterolateral nucleus of the thalamus, and the parvicellular part of the ventral 466 posteromedial nucleus of the thalamus differ and belong to two motifs with the number 8 and $467 \quad 7$ (for the parvicellular part) in Figure 5.

\section{Comparison of DRNs to in-vivo brain dynamics after stimulation}

469 To compare the simulated and empirical spatiotemporal dynamics of the brain activity after 470 focal stimulation of the isocortex, we used wide-field voltage-sensitive dye (VSD) imaging combined with optogenetic focal stimulation of the cortical areas (Figure 7, panel A). This technique allowed us to capture the brain activity over a large portion of the cortical mantle (Figure 7, panel B) while an arbitrary point on the cortical surface was stimulated (Figure 7, panel C). We qualitatively observe a large similarity between simulated and empirical patterns of brain activity evoked by the focal stimulation of the retrosplenial (RSC) and visual areas (see Panels A in Figure 8 to Figure 10). This similarity was described by the overlap of the maximally activated patterns of cortical regions after focal stimulation (see Panels $\mathbf{B}$ in Figure 8 to Figure 10). The spatial organization of these patterns is not trivially linked to the stimulation focus and shows characteristic and reproducible large-scale activations across the measured spatial window. Both empirical and simulated data suggested that the brain activity evoked by the focal stimulation could be predicted by the structural connectivity between regions. For example, focal stimulation of the RSC led to the activation of the visual and midline areas, which are strongly connected to RSC. However, our empirical and simulated data did not agree when we compared the simulated and empirical spatiotemporal patters of brain activity evoked by the stimulation of sensory areas other than visual ones. Here we did not observe a great amount of overlap in the set of activated cortical regions after focal stimulation of such areas.

488 Moreover, the temporal order of activation cortical regions after focal stimulation showed 489 some level of similarity (see Panels D in Figure 8 to Figure 10, and Supplementary Figure 1 490 to Supplementary Figure 3). We observed that the temporal order of activation in the 491 simulated data was preserved up to one or two transpositions compared to the empirical data.

492 It suggests that our model assumptions can fairly explain the flow of information in the 493 cortical networks. 


\section{Structure and division of the mouse brain model}

496 The model is based on the Allen Brain Atlas (ABA), which compiles tracer injections, 497 selectively sampling mice brain volumes, to map axonal projections of different areas within 498 the adult mouse brain (see Figure 1). The database of the Allen Brain Atlas was spatially 499 sampled with a resolution of $50 \mu \mathrm{m}$ (voxel of $50(\mu \mathrm{m})^{3}$ isotopically sample the volume). In 500 this way, the model distinguishes between 512 distinct brain areas. The smooth surface of the isocortex was reconstructed from the ABA, approximated by a regular mesh of 27,554 triangles with 41,524 edges ( $88 \mu \mathrm{m}$ is the length of the shortest edge, $190 \mu \mathrm{m}$ is the longest edge, with a mean edge length of $125 \mu \mathrm{m}$ ) between 13,972 vertices (Figure 11), and spatially divided into 84 cortical areas given by the ABA. The division of the reconstructed isocortical surface into areas, that is, the parcellation was performed by (i) extending the normal vectors of each vertex to about $200 \mu \mathrm{m}$ inside the volume of the isocortex, (ii) determining the 5 closest injection sites, and (iii) evaluating their classification to an area of the isocortex in the ABA. The isocortical parcellation was furthermore corrected for holes and isolated/mismatched vertices. The 512 distinct brain areas contain the 84 isocortical areas (42 per hemisphere) and 428 (non-isocortex) areas composing the olfactory areas, the

511 hippocampal formation, the cortical subplate, the cerebral nuclei, the thalamus, the

512 hypothalamus, the cerebellum as well as nuclei in the midbrain, the pons, and the medulla

513 (see Table 1 for the division, Table 2 and Table 3 for a complete list of area names). These

514428 sub-isocortical areas are lumped in the model to a point in physical space (i.e., centroid).

\section{Short-range structural connectivity}

516 The short-range connectivity comprises axonal projections within the isocortex (e.g., in a 517 brain region) over distances of $\mu \mathrm{m}$ up to $\mathrm{mm}$ on the surface (Braitenberg and Schüz, 1998).

518 These short-range connections were assumed to be homogeneous throughout the isocortex, 519 which connectivity decreasing with distance (Braitenberg and Schüz, 1991). In the model, the 520 so-called homogeneous structural connectivity (SC) resembles short-range connections 521 linking vertices of the isocortical mesh within an area, and between areas if they are spatially 522 close to one another with a connection probability following a Gaussian function and 523 decreasing with distance. Note that the homogeneous SC concerns the isocortex. Other brain areas are not directly linked via the homogeneous SC. Because the homogeneous SC represents an approximation of short-range connectivity, the spatial range of the Gaussian 
steps of $100 \mu \mathrm{m}$. The spatial cut-off range for short-range connections was eight times the standard deviation of the connectivity kernel $8 \sigma$ (Spiegler and Jirsa, 2013). This gives six configurations of the homogeneous SC.

\section{Long-range structural connectivity}

531 The long-range connectivity comprises axonal projections of distant brain areas. This type of 532 structural connections is estimated by the ABA between the 512 distinct brain areas from 533 adult mouse brains. The weights of the connectivity are obtained by the ratio of the projection 534 density to the injection site volume (Oh et al., 2014). Note that the ABA provides directional 535 information connectivity in the connectivity. The connectivity of the lateral geniculate nucleus to the primary visual area, for instance, weighs more than the connectivity of the primary visual area to the lateral geniculate nucleus. In the model, the so-called heterogeneous structural connectivity (SC) resembles long-range connections linking the brain areas. The heterogeneous SC links all the vertices of an area with the vertices of another area. Note that the 428 non-isocortical areas represent a node in the large-scale brain network. Connections between these nodes are point-to-point connections. Connections between an area of the isocortex and on of the 428 non-isocortical areas are links between all vertices of the isocortical area and the node of the non-isocortical area. Note that within the isocortex, neighboring areas are able to exchange information via the homogeneous SC within the cortex and via the heterogeneous SC leaving and entering the isocortex. At each local node (brain area) activity arrives and exits via afferent and efferent projections with an experimentally derived strength that indicates how strong a node collects and distributes activity - in other words, how well node is embedded in the network. The graph-theoretic measures, that is, in-strength (afferent projections and arriving activity) and out-strength (efferent projections and exiting activity) are computed given the experimentally derived large-scale heterogeneous SC. In addition, the in-degree (number of afferent connections) and out-degree (number of efferent connections) are computed. Figure 12 and Figure 13 list the top 10 brain areas, indicating that the hypothalamus is the most embedded brain structure in

554 the mouse brain model based on the ABA. The hypothalamus receives the strongest input in the mouse brain model and the cerebellum the weakest. The graph-theoretic measures also indicate that the subcortical structures are weakly connected with the isocortex tough ample connections exist. The graph-theoretic measures also indicate that the subcortical structures 
560 but rather emphasizes that the isocortex can be functionally autonomous as well under particular circumstances (certain brain states).

562 Though the tracer injection techniques underlying the ABA allow for directional information 563 of connectivity, the analysis of the weight matrix of the ABA structural connectivity reveals a 564 high symmetry, thus dominant bi-directionality of connectivity. Two measures were used for estimating the symmetry in the connectivity weights (see Materials and Methods). Both measures, $Q_{0}$ and $Q_{1}$ ranging between 0 (if symmetric) and 1 (if asymmetric and antisymmetric respectively) and indicate a high symmetry in the long-range structural connectivity reconstructed from the ABA with $Q_{0}=238.2871 \times 10^{-3}$ and $Q_{1}=226.6417 \times 10^{-}$ ${ }^{3}$. The tracer experiments compiled in the ABA mostly relate the spatial diffusion of the tracer to the injection site in the right brain. The connectivity is consequently left/right symmetric in the mouse model. For that reason, results are shown for one brain site though the model simulations where performed using both left and right hemispheres and the interhemispheric connections. For a sanity check, the simulated activity was correlated between the same area in the left and right brain and confirmed the left/right symmetries imposed by the area.

\section{Conduction speed and transmission delays}

576 The axonal conduction speed is determined by the axonal diameters as well as the degree of myelin. Both the axonal diameter and the degree of myelination vary throughout the mice brain (Wang et al., 2008; Foran and Peterson, 1992). Compared to larger mammalian brains as in humans, axons are less myelinated in the brains of mice, and if so, the axons are shorter (see, for example, Wang et al., 2008). The axonal conduction speed in mice is in the range of $0.2 \mathrm{~m} / \mathrm{s}$ (Wang et al., 2008) for unmyelinated axons with an average axonal diameter in mice of $0.3 \mu \mathrm{m}$ (Braitenberg and Schüz, 1998). The axonal conduction speed for myelinated axons is in range of $0.3 \mathrm{~m} / \mathrm{s}$ and $3.5 \mathrm{~m} / \mathrm{s}$ (Wang et al., 2008; Swadlow and Waxman, 2012; Salami et al., 2003). Length and the conduction speeds of axons cause time delays in the transmission of information processing. In contrast to human brains, the conduction via myelinated axons in mice is slower and more similar to unmyelinated axons (Wang et al., 2008). Moreover, the ratio of myelinated axons to unmyelinated axons is smaller in mice brains compared to human (Wang et al., 2008). Myelinated axons are shorter in mice brain compared to human (Wang et al., 2008). Note that the injection techniques underlying the ABA do not provide an estimate of the axonal lengths. The Euclidean distances between brain areas could be used as a proxy to estimate axonal lengths. Another possibility is given by the availability of diffusion tensor MRI data, from which the axonal tracts can be estimated and a connectivity matrix, similar to 
provide information about directionality but mouse individual connectivity information. With the availability of diffusion tensor MRI data of mice, the ABA connectivity could be coregister with the diffusion tensor connectivity, and thus provide information about axonal lengths. Following the reasoning of Buzáki and Wang (2012) the local brain activity, that is, of neural populations shows gamma oscillations $(30-90 \mathrm{~Hz})$ with cycle durations between 11 ms and $33 \mathrm{~ms}$. Considering that the average length of axonal tracts between lateral areas of one isocortex and contralateral isocortical areas in mice ranges between $20 \mathrm{~mm}$ and $40 \mathrm{~mm}$ (Schüz et al., 2006) and assuming that the time scales of transmission delays have to be similar to the characteristic time scales of local brain activity, result in an effective conduction speed smaller than $3.6 \mathrm{~m} / \mathrm{s}$ for the extreme, that is, the longest axon $(40 \mathrm{~mm})$ and the fastest local activity $(11 \mathrm{~ms})$. This estimation is in line with the range of conduction speed found in myelinated visual cortical neurons through the corpus callosum in mice (Swadlow \& Waxman, 2012). Most of the cortico-cortical homolateral connections in mice are shorter than $10 \mathrm{~mm}$ (e.g., Braitenberg and Schüz, 1998, Chapter 26, report $7 \mathrm{~mm}$ for the longest distance to the tracer injection site in a planar view on the isocortices with two modes, at about $2 \mathrm{~mm}$ and at about $3.5 \mathrm{~mm}$ ). Taking the same approach, this results in effective conduction speed values smaller than $1 \mathrm{~m} / \mathrm{s}$ for a maximum length of $11 \mathrm{~mm}$ divided by $11 \mathrm{~ms}$ to affect fast (90 $\mathrm{Hz}$ ) gamma oscillation (the upper bound of the speed for the longest distance to the tracer injection site and the two modes of distance to the tracer injection site, see, Braitenberg and

613 Schüz, 1998, Chapter 26, are $0.6364 \mathrm{~m} / \mathrm{s}=7 \mathrm{~mm} / 11 \mathrm{~ms}, 0.1818 \mathrm{~m} / \mathrm{s}=2 \mathrm{~mm} / 11 \mathrm{~ms}$, and $6140.31818 \mathrm{~m} / \mathrm{s}=3.5 \mathrm{~mm} / 11 \mathrm{~ms}$ ). Transmission delays are assumed to hardly affect intrinsic 615 local activity, that is, gamma waves, which are most apparent at a frequency of $>40 \mathrm{~Hz}$ across the brain. If we assume a modest spread of $3 \mathrm{~m} / \mathrm{s}$ and $10 \mathrm{~mm}$ length, then this results in time delays of up to $3.333 \mathrm{~ms}$. This is short and may not play a role in the mouse, but remains limit, especially if the speed can be slowed down by a fact of 2-3. Considering harmonic oscillations in the brain, the time delay can be effective on a scale smaller than the characteristic time constant of the intrinsic frequency (that is the period of a cycle) and expected to be most effective around a quarter of a full cycle (and multiple of half a cycle) because of the gradients, which results in effective time delays around $6.25 \mathrm{~ms}$ for $40 \mathrm{~Hz}$ and $2.77 \mathrm{~ms}$ for $90 \mathrm{~Hz}$. This means that a conduction speed of about $1 \mathrm{~m} / \mathrm{s}$ is effective for $40 \mathrm{~Hz}$ oscillations if the majority of connections is about $6.25 \mathrm{~mm}$ long (and $2.77 \mathrm{~mm}$ for $90 \mathrm{~Hz}$ ).

625 Short time delays affect mainly fast oscillations but not necessarily slow oscillations and long

626 time delays affect slow oscillations more than fast oscillations. In this sense, we have 627 provided each network node with simple dynamics that allows it to respond to stimulation and 
628 incoming input from other brain regions with a damped oscillation in the gamma range (at about $42 \mathrm{~Hz}$ ), see Figure 2.

630 The conduction speed for white-matter fiber tracts is assumed in the model to be $1 \mathrm{~m} / \mathrm{s}$. Given

631 the distances between the brain areas from the ABA, the transmission delays result from the 632 division of the mean connection lengths by the conduction speed (see Figure 14). First of all, 633 the histograms in Figure 14 do not give indications for bimodality as reported by Braitenberg 634 and Schüz, 1998 in Fig.62, Chapter 26, though the technique is similar and the distance 635 measurement is slightly different. However, after dividing the entire mouse brain into areas 636 (e.g., left/right isocortex) and subareas (e.g., left/right barrel cortex; following the ontology of $637 \mathrm{ABA}$ ) and investigating intra-hemispheric connections (e.g., among left isocortical areas) and 638 inter-hemispheric connections (e.g., between left and right isocortical areas) we can confirm 639 the second peak in the histogram whose significance is obscure (see Figure 14 panel B). This 640 second peak basically reflects the long commissure connecting the two hemispheres such as 641 the corpus callosum. Because the number of connections within the isocortex is similar to 642 those between left and right isocortical areas (Table 4), the effect of the two peaks in the 643 histogram for intra- and inter-hemispheric connection lengths is not visible in the histogram 644 of all isocortical connection lengths. The same is true for all the connections in the entire 645 mouse brain model (see Figure 14 panel A). The descriptive statistics of the connection 646 lengths are summarized in Table 5.

\section{Local areas and their dynamics}

648 Each of the 512 brain areas is assumed to intrinsically perform an oscillation with a natural 649 frequency in the gamma range (at about $42 \mathrm{~Hz}$ ). This rhythm in the gamma band accounts for 650 local activity, such as a coordinated interaction of excitation and inhibition (Buzsáki and 651 Wang, 2012), which is not physiologically, but phenomenologically modeled here 652 (Palmigiano et al., 2017). Having said that, the damping factor $\gamma$ determines the local 653 excitability (i.e., the critical distance to the Andronov-Hopf bifurcation), and is thus a 654 surrogate for the ratio of excitation and inhibition at each brain area. A two dimensional (2D) 655 model (i.e., consisting of two state variables: mean postsynaptic potential and current) is used 656 to provide the temporal dynamics of each brain area. In case of the isocortex, where brain 657 areas, that is, isocortical areas are spatially extended, each mesh vertex holds a 2D-element. 658 The 2D-model is equally parameterized for all brain areas by $\gamma=1.21, \varepsilon=12.3083$. This 659 means that without any links between the brain areas, local behavior is identical. Without any 660 stimulation (no noise, no time-variant input) the activity is constantly zero. The local response 661 to stimulation is a damped oscillation in the gamma frequency range. Because the long-range 
662 connections in the structural network of the mouse brain are heterogeneous (see Figure 14), 663 subsequently emerging brain function (and dysfunction) is shaped by the brain structure 664 through the network interactions and, hence, the local response can be highly specific to a 665 brain area though the areas are equally parameterized.

State system and its parameterization

667 The modeling approach to generate brain activity in The Virtual Brain (TVB) involves three interacting systems, namely, concerning the inputs, the dynamics and the observations. Where the input system allows for interventions and the observer system specifies the measurement modalities, the dynamical system generates the brain activity and constitutes brain states. The physical and spatiotemporal properties of actions to brains determine the input to TVB (compare, for example, focal brief direct electrical stimulation, transcranial direct current stimulation and retinal stimulation). In general, models are agnostic about the action and hence have the potential to integrate and moreover to discuss the effects of different modalities of action such as of sensory, direct electrical and transcranial stimulation but also blocked arteries and changes of myelin sheaths. The input system relates an action to the state variables of TVB (e.g., postsynaptic potentials and currents) causing a set of events (over a relative short time scale, for example, event-related potentials (e.g., Shah et al., 2004) and event-related (de)synchronization (Pfurtscheller \& Lopes da Silva 2001), and long-term effects such as necrosis and changes in apoptosis and autophagy). Such events may be symptomatic for medical conditions and diseases such as ischemic stroke (as a result of blocked artery) and multiple sclerosis (due to damage of myelin sheath). The dynamical system comprises the variables with which the brain activity is modeled. The model is a network of 14,400 nodes $(13,972$ vertices for the 84 spatially extended areas on the isocortical surface, and 428 nodes, each for a non-isocortical brain area). Links between brain areas via the homogeneous SC (short-range connections) and the heterogeneous SC (long-range connections) are assumed to be excitatory. Inhibition is implicitly present in the gamma oscillator representing local activity of each brain area, which is distinguishable in the action (excitatory or inhibitory) of their projections. To our best knowledge, however, neither a complete nor an extensive classification is currently available for the characterization of all 512 considered brain areas in the model (e.g., to excite or to inhibit synaptically connected area). The weights of connections are normalized to maximum unity in-strength of a brain area so that the network is segregating and integration information, where the energy of activities decays in the network. In other words, the network cannot amplify activity by 
example, Spiegler et al., 2016). Consequently, the state of the system is spanned by 28,800 state variables. Note that the local behavior was set to a subcritical working point as suggested by many authors within the resting state literature (Deco et al., 2011; Fagerholm et al., 2015). Subcriticality is here represented by a stable focus close to instability, showing damped oscillations in response to perturbations. The specific connections to a given network node (brain area) and the energy that they transfer from connected nodes change the operating point of the local node towards the critical point (i.e., Andronov-Hopf bifurcation) at which the decay time of a stimulation response increase and the node starts performing a selfsustained oscillations (in absence of any stimulation). The brain model is however parameterized that none of the applied stimulation led to a self-sustained oscillation (i.e., limit cycle). Consequently, the brain network segregates and integrates information, where the energy is dissipated by the local intrinsic dynamics. The model parameters are the six configurations for the spatial range of the homogeneous SC on the isocortical surface and the ratio of homogeneous to heterogeneous SC. The brief action of the stimulation is related to the set of nodes spanning a given brain area by the input system. The input system adds the input describing brief focal stimulation of brain areas to the postsynaptic potentials (PSPs).

Observer system for voltage-sensitive dye (VSD) imaging

The physical forward solution is captured by means of an observer system links, which links the variables of the state system (here, the mean postsynaptic potential and current) for the 2D-model at each network node, to an experimentally observable measure. To compare the activity in the isocortex due to stimulation in the model and in the experiment during the voltage-sensitive dye (VSD) imaging (Mohajerani et al., 2013) the simulated brain activity is projected onto the plane that reconstructs the focal plane from which the camera setup captures the VSD images. VSD imaging measures the emitted light from the superficial layers of the isocortex stained with VSD molecules as a function of the neuronal membrane potential. The results show that the temporal fluctuations in the light emission are correlated with the electrophysiological measures such as local field potentials (Mohajerani et al., 2013). This suggests that VSD imaging reflects postsynaptic potentials (PSPs) of neural populations. Consequently, we establish a direct link between the state variables of neural activity and the VSD imaging data by equating the first variable of the 2D-model with the PSP of a local population and the second variable, which is the temporal derivative of the PSP, with the current. The PSP of a local population can be linked to the VSD imaging. However, the VSD imaging projects only the brain activity within a limited spatial range around the focal plane (i.e., the depth of field) intersecting the isocortex onto a plane (image sensor) regardless of the 
curvature of the isocortex in physical space. In the VSD imaging data, isocortical brain areas were identified by the early response to different sensory and peripheral stimulation (i.e., whisker, auditory, forelimb, hindlimb, and visual). These locations were used to find a projection plane (size given by the camera sensor used for the VSD imaging) in the model that best corresponds to the VSD imaging data (see Figure 15). This mapping provides then a direct link of the local PSP in the model to the measured VSD images. The isocortical areas, captured by the VSD imaging setup (see, for more details, Mohajerani et al., 2013), are listed in Table 6. Simulated and measured activity can thus be compared on the imaging level as well as by the region mapping.

\section{DISCUSSION}

739 Brain network dynamics can be systematically explored only in silico. To explore the mouse brain dynamics in silico, we built a high-resolution whole mouse brain model. The model structure is based on the database Allen Brain Atlas (ABA) comprising tracer studies. We investigated the network behavior by assuming simple oscillatory but canonical dynamics at each brain region, that is, a network node. Each region had the same local dynamics, only differentiated by the ABA connectome from one to the other. We simulated the whole-brain mouse model on a computer using the neuroinformatics platform The Virtual Brain (TVB). To test whether the mouse brain activity can be exclusively inferred from the underlying connection topology, we performed graph theoretic analysis of the model structure. The results show that the overall performance of the 13 graph-theoretic measures is rather poor. The best prediction of the simulated brain responses to focal stimulation and the extracted dynamically responsive networks (DRNs) resulted from comparing the rank of the activity with the rank of connectivity strengths of the direct projection from the stimulation site, where a high correlation indicates that the energy induced by the stimulation spreads into areas of the brain that are directly connected to the stimulation site. The rank correlation is weak but significant for most connectivity parameter configurations. We compared measured and simulated brain activity using the observer model for (virtual) VSD imaging. For exploring the mouse brain dynamics in silico, we systematically varied three model parameters, namely (i) the ratio to which extend brain areas can interact over short- and longranges, (ii) the range of short connections that are homogeneous on the isocortex, and (iii) the location of the focal stimulation. The extent to which information is processed over short- or long-range SC is unclear. The results show that both SC types of short- and long-range connections in the mouse model act as spatiotemporal filters of local dynamics in the emergent functional network. The results (Figure 3) do not support evidence for a 
reorganization of dynamics due to interplay between both types of SC in the mouse as compared to human models (Spiegler et al., 2016). We performed analyses of the brain responses to stimulation. The focal stimulation is closely related to direct electrical, sensory and optical stimulation. We identified consistent spatial motifs of mouse brain activity called dynamically responsive networks (DRNs) using dimension reduction methods. We found the experimentally known functional networks back in the DRNs. Interestingly, the salience and the default mode network spanned several motifs indicating the salience and default mode network to be a junction for functional networks. We furthermore investigated the spatiotemporal organization of brain activity following sensory pathways. The model structure reflects the sensory systems, namely: the auditory pathways, the visual pathways, the whisker pathways and the pathways for the limbs. Finally, with the developed large-scale mouse brain network model we had, for the first time, the chance to systematically investigate sensory pathways and, especially, the processing of sensory data. We showed that the insilico brain responses resemble in-vivo brain dynamics after stimulation. In the following we discuss the results in more detail.

Modeling results

The structure of the model (Figure 1) based on the database of the Allen Brain Atlas comprising tracer studies was coregistered with the anatomy of the mouse brain (see Oh et al., 2014). We spatially sampled the brain volume given by the ABA and obtained a network model composed of 512 distinct brain areas. The model incorporates the smooth surface of the isocortex that we reconstructed from the $\mathrm{ABA}$ using a regular mesh of 27,554 triangles with 41,524 edges between 13,972 vertices (Figure 11) and the spatial extent of the 84 cortical areas given by the ABA. The 512 distinct brain areas contain the 84 isocortical areas (42 per hemisphere) and 428 non-isocortex areas (see Table 1 for the division, Table 2 and Table 3 for a complete list of area names). These 428 sub-isocortical areas are lumped in the model to a point in physical space (i.e., centroid). From these numbers, it is evident that the isocortex constitutes only a small part of brain areas considered in the model (and in the ABA). However, the isocortex is the structure from which the here considered wide field VSD imaging captures activity from 26 isocortical areas in the ABA. Thus we often observe only from a small fraction of interconnected areas (here 26 observables vs. 512 brain areas in the model). Here it is important that the spatial resolution in the model coincides with the resolution of the model. Although the resolution of the brain geometry (e.g., the isocortical mesh) agrees with the measurements (e.g., VSD imaging), the quality of the modeling highly depends on the division of the brain into areas (parcellation) and with that the resolution and 
reconstruction of connections (Proix et al., 2016). The version of the ABA that we have used to construct the model, for instance, the barrel field of the primary somatosensory areas is summarized by one single area (see Table 2). A distinction of the barrels in the barrel field of

800 the primary somatosensory areas would improve to distinguish lower and upper limb 801 (especially hind and forelimb), see Figure 6 panel E. The model can be improved by using not 802 only the new ABA model (https://www.biorxiv.org/content/early/2018/04/01/293019) but also by incorporating

other databases (e.g.,

804 http://www.mouseimaging.ca/research/mouse_atlas.html; Dorr et al., 2008; www.MouseConnectome.org).

806

807

808

809

810

\section{Modeling brain dynamics and limitations}

We assumed a natural frequency of $42 \mathrm{~Hz}$ at each network node and $1 \mathrm{~m} / \mathrm{s}$ of conduction speed - both within biologically plausible ranges (see section 'Conduction speed and transmission delays' and section 'Local areas and their dynamics' in the Results). By this simple dynamics at each node we probed the experimentally derived large-scale brain network to produce functionally relevant organizations in the activity by stimulation. If the model equipped with this simple dynamics does not show functionally relevant organizations in the simulated brain activity then the model misses relevant complexity. The experimentally derived large-scale brain network may miss essential features (perhaps the average brain structure is not sufficient and averages out important components) and better data needs to be provided (better estimates for the connectivity strengths). The behavior in the large-scale brain signals (e.g., M/EEG and functional MRI) associated with brain function and dysfunction are perhaps epiphenomena at the large scale of the brain and actually originate from processes on a micro- and meso-scale (rather than throughout the interaction of brain areas) that are reflected onto the large scale of the whole brain and its functional measurements. Another point is the local dynamics itself. The local model for the simple dynamics that we use here incudes a surrogate parameter for the ratio of excitation and inhibition. However, this model is generic and does not represent a particular local structure. If this model is not sufficient, biologically informed models are available. The local dynamic models can be biologically informed about the local circuitry and specific activities (e.g., certain rhythms). Local circuitries are sketches of characteristic elements (e.g., projection neurons, interneurons) and their connections involved in relevant processes (e.g., excitation/inhibition) based on morphological and physiological arguments. Using such biologically informed models increase the repertoire of dynamics at a node by their local 
830 circuitry and are thus meso- and microscopic descriptions. It is worthwhile to mention that the complexity in the behavior is linked to the complexity of the structure. Complex behavior

832 (e.g., occurrence of spike-wave complexes or epileptic discharges in distant brain areas) 833 indicates the complexity of systems and therefore cannot be embedded in simple systems. If 834 brain dynamics are provided large-scale models are able to provide plausible than the large835 scale network provides entry points for interventions, useful for diagnostics and therapy 836 among others.

\section{DRNs and functional networks}

838 We performed a systematic exploration of brain dynamics via focal stimulation to extract the 839 DRNs. The DRNs are constrained by the local model dynamics and the connection topology. 840 The interplay of these factors imposes conditions on the dynamic repertoire of a given 841 network, independent on the details of how these factors are physiologically realized. To be 842 specific, there is a multitude of ways to generate a particular behavior of a neural population 843 (Marder \& Taylor 2011) such as an oscillation, but once established, then it does 844 communicate via the connectivity with other regions and the actual physiological 845 underpinnings play no role anymore (unless new factors arise such as spike rate adaptation, 846 for instance). From this perspective, the DRNs fundamentally reflect the functional dynamic 847 repertoire of a network given a particular connectivity. The DRNs and the mapping to 848 stimulation allows for a coordinated interaction with functional networks using different types 849 of stimulation (e.g., sensory and transcranial). The DRNs show characteristic motifs. Each 850 motif is a spatial distinct organization of brain activity and composed by three parts. We 851 identified five different spatial patterns in the parts that entangle the motifs. The presence of a 852 pattern in several motifs suggests that the brain is able to phrase more than one motif (thus more than one DRN) at a time. The experimentally known functional networks, especially the default mode network (and the salience network) are reflected in parts of these motifs.

855 With the present modeling study, we extracted the brain motifs, the possible expressions, and 856 how it is organized. The spatial patterns tell us which brain area is active. We also describe 857 the themes by relating the patterns to experimentally known functional networks. The used 858 local model is simple and appropriate for investigating the large-scale spatiotemporal 859 organization of brain responses, which is the scope of this work. As a consequence of the mono-rhythmic behavior and the uniform parameterization of the local dynamic model, the simulated network dynamics show limitations in describing the various rhythms involved in the brain organization (i.e., neuronal firing and brain rhythms alpha to gamma). For the 
864 model has to allow the generation of more complex activity, which could be potential subject 865 to subsequent studies. The modeling is challenging and goes hand in hand with the debate 866 about the generation of brain activity such as rhythms and especially its spatial extent. The 867 risk in this kind of modeling work based on large-scale data (as with modeling based on 868 microscopy data, see Marder \& Taylor, 2008) lies in the overfitting (additional state variable 869 increase the degree of freedom and may increase the complexity of the model). One approach 870 would be to use the same simple local dynamic model for each node as we have done in this work but then to parameterize nodes differently accounting for the spatial differences in natural frequencies.

873

874

875

876

877

878

879

880

881

882

883

884

885

886

887

\section{Sensory pathways}

The sensory networks in Figure 6 are mainly based on textbook descriptions (e.g., Watson et al., 2011) and include the relevant structures given by the ABA. The sensory pathways can be described using the ABA. However, the ABA needs refinements to distinguish lower and upper limb (especially hind and forelimb), see panel E. Neither does the ABA include a division of the barrel field of the primary somatosensory areas. The networks in Figure 6 indicate information flows to areas that do not necessarily terminate in the primary sensory areas of the isocortex, such as the hypothalamic and midbrain targets of the retinal ganglion cells in in Figure 6, panel $\mathbf{A}$, and the midbrain nuclei related to whisker movements in Figure 6, panel D. These connections are well known and usually not discussed regarding sensory processing in textbooks. As a result, visual system and whisker system meet in the sensory related superior colliculus regarding eye and whisker movements. It is worth mentioning that the model is agnostic about the (sensory) information, meaning that nuclei may be activated but the object of processing (information) is less defined. The vast amount of studies about the physiology of nuclei allows attaching meaning to the modeling results. For instance, the hypothalamic nuclei and the nuclei in the midbrain (nucleus of the optic tract, olivary and posterior pretectal nucleus) receive input from the retina but are not directly part of the visual pathways running from the retina to in the primary visual cortices, see Figure 6, panel $\mathbf{A}$. The hypothalamic nuclei play however a role in the circadian timing system and the nuclei in the midbrain are known to be involved in eye movement coordination and reflexes.

Certain patterns in the auditory and visual pathway belong to the same cluster. This is most likely because of the spatial proximity of the inferior colliculus to the superior colliculus (sensory related). The resolution of the mouse tracer studies may be not good enough because of leakage. 
897 There is also overlap between whisker and limbs pathways. The barrel cortex (i.e., Left 898 Primary somatosensory area, barrel field (area 5)) is only separated from the limb areas (i.e., 899 Left Primary somatosensory area, lower limb (area 6) and Left Primary somatosensory area, 900 upper limb (area 8)) by an unassigned area, that is, Left Primary somatosensory area, 901 unassigned (area 10). Consequently, the parcellation might be misleading taking into account 902 that we are dealing with an average mouse brain compiled by numerous different brain of 903 (genetically the same mouse type). This may explain the uncertainty in the matching by errors 904 in the allocations of brain tissues.

905 Ventral posteromedial nucleus, Ventral posterolateral nucleus, and Posterior complex are 906 neighboring areas and part of the posterior nuclei of the thalamus. The grouping may indicate 907 an uncertainty and limitation of the data because of the proximity and the diffusion of the 908 tracer locally during the measurement technique and the agglomeration of different 909 experiments.

\section{Translation Human Animal}

911 With this study we adapted the focal stimulation protocol that we have used previously in912 silico for exploring human brain dynamics. Although the mouse brain is way smaller than the 913 human brain, the models are comparable in terms of number of nodes used to describe the 914 brain network (in 16,500 human vs. 14,000 in mouse). However, because of the different 915 brain sizes, brain areas, especially the subcortical areas are described in more detail in the 916 mouse brain than in the human (76 cortical areas and 116 thalamic areas in the human model 917 vs. 84 isocortical areas and 428 subisocortical areas in the mouse model). The mouse brain 918 model used in this study is most detailed in the subisocortical structures, namely cerebellum, 919 medulla, pons, midbrain, hypothalamus, thalamus, and cerebral nuclei). It does provide an excellent validation framework for the large-scale brain network modelling approach.

921 One of the main questions was to which extent information is processed via short- and longrange connections. The result in human was that the short-range connectivity is relevant for explaining experimentally known functional networks. By varying the spatial extent of shortrange connections and varying the ratio of short- to long-range connections we observed a reorganization of the DRNs and the brain dynamics. This reorganization of brain dynamics due to changes in the connectivity could not be found in the mouse model. The model suggests that the mouse brain dynamics are organized around 12 motifs (see Figure 5). These motifs are fairly stable in the mix of short-range and long-range connections (see Figure 3).

929 The motifs of the DRNs in both the mouse and the human model reflect functional networks.

930 Whereas the DRNs in human span a variety of functional networks (e.g., default-mode, 
memory, attention), the DRNs in mice cover mainly functional networks at rest such as salience, default mode, medial, and lateral networks and less the sensorimotor networks (only

933 the network of the frontal eye field).

934 Application of stimulation techniques is limited in human to transcranial and sensory 935 stimulation and intracranial stimulation in patients. In animals a battery of interventions is 936 available such as optogenetic tools and electrophysiology. These tools make validation

937 feasible and, moreover, the translation to human through modeling so highly important.

939 Stimulation paradigms are critical for the validation of any model paradigms, because 940 conceptually they take the system out of its attractor states and allow the sampling of their 941 dynamic neighborhood. The thus obtained information is significantly more predictive than 942 any resting state paradigms.

943 The simulated data after focal stimulation of the visual and retrospleial cortices match better 944 with the empirical data. We suggest that it is because the tracing experiments provided by the 945 ABA describe the structural connectivity of the visual network more accurately than that of 946 the sensory one. We believe that by incorporating a more accurate structural connectivity of 947 cortical networks, we can achieve a more realistic model, which is able to produce patters of 948 activity resembling the empirical data.

949 Beyond connectivity, there are other significant simplifications present in the network model 950 affecting its validity, when confronting with empirical data. The most obvious one, from the 951 network perspective, will be the so far assumed homogeneity of local network node 952 dynamics. It is well established that local brain region dynamics varies across the network in 953 terms of excitability (physiological rhythms measured in intracranial human EEG vary across 954 brain regions, see Bartolomei et al., 2017), frequency (posterior to anterior gradient of 955 eigenfrequency ranging from 10 to $40-50 \mathrm{~Hz}$ (e.g., Rosanova et al., 2009), and rhythmic 956 organization (burst activity such as in the reticular nucleus of the thalamus (see Marlinski \& 957 Beloozerova, 2014; Shermann, 2001); harmonic activity (for example, the alpha waves 958 originating from the occipital lobe, see Niedermeyer, 1997), others behavior such as the 959 activity change in suprachiasmatic nucleus of the hypothalamus course of the circadian 960 rhythm (see Welsh et al., 1995)). These factors are well known to determine the 961 synchronization behavior of networks, although their influence on spatiotemporal energy 962 dissipation and the creation of DRNs may be limited, because of the large-scale nature of 963 network propagation considered here. These are doubtlessly important factors, but 
964 connectivity will remain the most critical one, thereby leaving it open the extent to which 965 connectivity details are functionally expressed.

966

967

968

969

970

971

972

973

974

975

\section{Conclusion}

The full-brain network perspective is very particular. In its essence, it decomposes the brain into a system composed of nodes and links, which is capable of spatiotemporal pattern formation. This view allows us to ask fundamental questions about stimulated pattern propagation, which is at the heart of information processing in the brain. As unsatisfactory the answers are physiologically (due to the nature of the approach), as astonishing is the validity and degree of detail, to which signal propagation following stimulation (sensory or focal) is explained without the need of fine tuning of parameters, further underwriting the fundamental nature of the insights gained here. We have essentially mapped out all the network signal propagation pathways supported by the mouse brain.

\section{MATERIALS AND METHODS}

In order to perform exploration of brain dynamics in silico we model the mouse brain using the large-scale brain model, consisting of the geometry and the structural connectivity combined with local dynamics. We performed simulations of the whole-brain network model, decomposed the brain response after focal brain stimulation, and extracted the dynamically responsive networks (DRNs). By performing statistics, we tested the predictability of the DRNs by the structural connectivity and compared experimentally known functional networks with the DRNs. Furthermore, we investigates the integration of stimulation and response along sensory pathways into the whole brain network. Finally we compared simulated data and voltage sensitive dye imaging and during in-vivo sensory and optogenetic stimulation in the mouse brain. The following sections provide a detailed description of each step:

\section{Modeling the mouse brain}

Using The Virtual Brain platform (Melozzi et al., 2017; Sanz-Leon et al., 2013, 2015; http://thevirtualbrain.org/), we triangulate the smooth surface of the isocortex using a regular mesh of 27,554 triangles with 41,524 edges ( $88 \mu \mathrm{m}$ is the length of the shortest edge, $190 \mu \mathrm{m}$ is the longest edge, with a mean edge length of $125 \mu \mathrm{m}$ ) between 13,972 vertices (Figure 1, Panel A-B, and Figure 11), distributed across 84 cortical areas given by the Allen Brain Atlas (ABA; http://connectivity.brain-map.org) (Figure 1, Panel C). Each area of the isocortex contains between 69 and 667 nodes in the mesh (Table 1 to Table 3) and, because the mesh is 
996 regular, the number of nodes indicates the size of an area in the ABA. In addition to the areas in the isocortex, the model includes 428 subisocortical areas. The division of the 998 reconstructed isocortical surface into areas, that is, the parcellation was performed by (i) 999 extending the normal vectors of each vertex to about $200 \mu \mathrm{m}$ inside the volume of the 1000 isocortex given by the ABA, (ii) determining the 5 closest injection sites, and (iii) evaluating 1001 their classification to an area of the isocortex in the ABA. The isocortical parcellation was 1002 furthermore corrected for holes and isolated/mismatched vertices.

1003 To connect nodes with each other, we distinguish homogeneous from heterogeneous SC 1004 (Figure 1, Panels D-F). The homogenous SC (of short-range connections) links nodes within 1005 an area, and between areas if they are spatially close from one another with a connection 1006 probability decreasing with distance (Braitenberg and Schüz, 1991, 1998) (Figure 1, Panels D

1007 and F). The heterogeneous SC (of long-range white matter tracts) links all the nodes of an 1008 area with the nodes of another area (Figure 1, Panels D and E), based on known anatomical 1009 atlas of the mouse brain, that is, ABA. Neighboring areas are able to exchange information 1010 via the homogeneous SC within the cortex and via the white matter tract, that is, 1011 heterogeneous SC (e.g. Area 2 with Areas 1 and 3 in Figure 1, Panel D).

1012 Each vertex point is a network node holding a neural mass model connected to other nodes 1013 via the homogeneous SC and heterogeneous SC. When an area is stimulated, all the nodes of 1014 this area are simultaneously activated and then the stimulation-induced activity in each node 1015 decays differently according to the activity in the surrounding via short-range connections 1016 (i.e., homogeneous SC) and remote nodes via long-range connections (i.e., heterogeneous 1017 SC). The ability to drive the network does not depend on the number of nodes within an area, 1018 because the heterogeneous SC transfers the mean of the activity in all the nodes within an area 1019 to all the nodes in another areas.

1020 We consider this ratio of homogeneous SC to heterogeneous SC as a degree of freedom and 1021 perform a parametric study (see Jirsa and Kelso, 2000; Qubbaj and Jirsa, 2007, 2009 for 1022 systematic studies with two-point connection). The ratio has been estimated. For instance, 1023 Braitenberg and Schüz (1998) assessed that pyramidal cells have synapses in equal shares 1024 from long-range and local axons. However, the ratio of homogeneous SC to heterogeneous 1025 SC mainly depends on the resolution of the used geometrical model of the cortex, with that 1026 the representation of the SC, and the network node description (e.g., canonical model, neural 1027 mass model), which is able to incorporate local connectivity (see, for example, Spiegler and 1028 Jirsa, 2013 for more detail). At the extremes, (i) $0 \%$ of heterogeneous SC (thus $100 \%$ of 1029 homogeneous SC gives two unconnected cerebral hemispheres with locally but 
1030 homogeneously connected nodes) only allows activity to propagate locally from a cortical stimulation site, and (ii) 100\% of heterogeneous SC (thus 0\% of homogenous SC gives 512 purely heterogeneously connected brain areas with locally unconnected nodes) only allows activity to travel long distances with time delays via white matter fiber tracts.

1034 Furthermore, since the spatial range of homogeneous SC is not known (Spiegler and Jirsa, 2013), we also consider it as a parameter varying between $500 \mu \mathrm{m}$ and $1000 \mu \mathrm{m}$. We then systematically stimulate each of the 512 areas with a large range of parameter values (for the ratio and the spatial range), resulting in a total of all 18,432 simulation trials. Brain dynamics at rest have been found to operate near criticality (Ghosh et al., 2008; Deco et al., 2011, 2013, Spiegler et al., 2016). Near-criticality is defined as a system that is on the brink of a qualitative change in its behavior (Shew and Plenz, 2013). The proximity to criticality predicts that the brain's response to stimulation will primarily arise from structures and networks that are closest to instability. Activities in those networks require the most time to settle into equilibria after stimulation, and are associated with large-scale dependencies and scale invariance (Haken, 1978). This would be consistent with the center manifold theorem, which states that a high-dimensional system in a subcritical state will converge on a lower dimensional manifold (here few networks) when the system is stimulated. Consequently, we equally set each node in the brain network model to operate close to its critical point, where the network shows no activity without stimulation. We use the stable regimen of each network node (i.e., stable focus) to stimulate a given area in the direction of its instability point (i.e., supercritical Andronov-Hopf bifurcation) and induce characteristic energy dissipation through the brain network. The dissipation of energy will be constrained by the homogeneous SC and heterogeneous SC, the associated signal transmission delays, and the local dynamics at the network nodes. In the network model, the operating point of every node, when disconnected from the network, is at the same distance from its critical point, that is, the supercritical Andronov-Hopf bifurcation (Figure 2, Panel A). If the critical point is reached,

1056 the node enters into a constant oscillatory mode. In the network, the SC (incl. time delays) 1057 determine the alteration of the working distance to the critical point at each node in time by weighting and delaying the incoming activity from other nodes in the network. Hence, network metrics of the SC such as the in-strength, that is, the sum of weights of incoming ties to a node may indicate the distance of a node's operating point to its critical point and thus criticality (Kunze et al., 2016). The network model, however, is set so that criticality is never reached, by normalizing the $\mathrm{SC}$ to unity maximum in-strength so that activity cannot be amplified through the SC. As a result, when a node is stimulated, the node operates closer to 
1064 the critical point and the response is in the form of a damped oscillation (Figure 2, Panel A).

1065 The closer a node operates to the critical point, the stronger the node's responses with high 1066 amplitude and long decay time (Figure 2, Panel A). The nodes are working near criticality 1067 (i.e., they get close to a change in behavior, which would be here a switch to a constant 1068 oscillatory mode, but never reaching it). Thus the response to the stimulation is transient, 1069 lasting a few milliseconds. The damped oscillation generated in one stimulated node is then 1070 sent via its efferent connections to its target nodes, triggering there, in turn, a damped oscillation (Figure 2, Panel B). If the network were mainly based on nodes connected in series, activity would decay very fast after the stimulation (Figure 2, Panel B). However, since the outgoing activity of a node can influence the nodes projecting back to it, recurrent systems appear (Figure 2, Panel C-D), which allow activity to dissipate on a much longer time scale. The evoked activity, after the initial decay, thus persists in the so-called responsive networks (Fig. 2c, d), which may reflect feedback loops and re-entry points in the SC. A dynamically responsive network acts on changes, for instance, due to sensory stimuli and random fluctuations in the network (flexibility), and outlasts the stimulation (criticality). The described network properties are illustrated in Figure 11 to Figure 14 as well as Table 4 and Table 5. The differences in the response stem from the proximity to criticality, which depends upon the SC (in particular the extent of recurrent networks), comprising the synaptic weights and the time delays (Figure 1). This behavior is predicted by the center manifold theorem, which is the mathematical basis for criticality (Haken, 1978).

1084 The directionality of the heterogeneous SC derived from the tracer studies provided by the 1085 ABA is assessed by two measures estimating the symmetry in the connectivity weight matrix. The first measure, $Q_{0}=[0,1]$, gives 0 if symmetric and 1 if asymmetric and reads:

$$
Q_{0}=\left\|\mathrm{A}-\mathrm{A}^{\mathrm{T}}\right\| /\left\|\mathrm{A}+\mathrm{A}^{\mathrm{T}}\right\|,
$$

1088 where $\mathrm{A}$ is the square matrix containing connection weights between the 512 areas, and $\|\mathrm{A}\|$ is the matrix norm. The second measure, $Q_{1}=[0,1]$ is defined as follows:

$$
Q_{1}=\left\|\mathrm{A}-\mathrm{A}^{\mathrm{T}}\right\| /\|2 \mathrm{~A}\|,
$$

1091 and gives $Q_{1}=0$ if the matrix A is symmetric and $Q_{1}=1$ if anti-symmetric. Both measures

1092 indicate a high symmetry in the weight matrix of the ABA SC and therefore a dominant bi1093 directionality of connectivity.

Large-scale brain model

1095 Dynamics of a vector field $\Psi(x, t)$ at time $t \in \mathbb{R}^{1}$ and position $x \in \mathbb{R}^{3}$ in space $\Omega$ are described 1096 by a delay-integro-differential equation: 


$$
\begin{aligned}
& \partial_{t} \Psi(x, t)=E(\Psi(x, t))-a_{I} I(x, t) \\
& +(1-\alpha) a_{L} \int_{L} \mathrm{~d} X^{\prime} \Psi\left(x-X^{\prime}, t\right) g\left(X^{\prime}\right) \\
& +\alpha a_{S} \int_{L} \mathrm{~d} X^{\prime} \Psi\left(x-X^{\prime}, t-\left\|x-X^{\prime}\right\| / v\right), \\
& \quad \times H(X) C\left(\left\|x-X^{\prime}\right\| / v\right) K^{\mathrm{T}}\left(X^{\prime}\right)
\end{aligned}
$$

1098 were $\partial_{t}$ is the derivative with respect to time, $t$. The input $I(x, t)$ allows the stimulation 1099 dynamics to intervene on a node. The operator $E(\Psi(x, t))$ locally links variables of the

1100 vector field. The scalar $\alpha$ balances the effect of the homogeneous SC and the heterogeneous $1101 \mathrm{SC}$ (first and second integral) on the vector field. The vectors $a_{I}, a_{L}$, and $a_{S}$ of factors relate 1102 the input $I$, and both types of SC to the vector field $\Psi(x, t)$. The kernel $g(x)$ describes the homogeneous SC. The field is time delayed due to a finite transmission speed $v$ via the heterogeneous SC given by matrix $C(x)$. The vectors $H(x)$ and $K(x)$ establish the links between the heterogeneous SC and the targets and the sources. Note that the transmission speed enters the second integral concerning heterogeneous SC. We assumed the transmission via the homogeneous SC (first integral) to be instantaneous, which reduces the computational expenses, in order to perform the parameter study. The spatial and temporal aspects of the model are described in more detail in the following two subsections.

Geometry and structural connectivity (SC)

1111 The spatial domain $\Omega=\left\{L_{1} \cup L_{2} \cup S\right\}$ separates both cerebral hemispheres $L=\left\{L_{1} \cup L_{2}\right\}$ :

1112 left, $L_{1}$ and right, $L_{2}$, from subisocortical areas $S$, that is, $\cap \Omega=\varnothing$. Two open surfaces describe 1113 the geometry of the left and right isocortex $\left(L_{1}\right.$ and $\left.L_{2}\right)$ by a regular mesh of 27,554 triangles with 41,524 edges between 13,972 vertices. The homogeneous SC follows a Gaussian distribution $g(x)=\exp \left(-x^{2} /\left(2 \sigma^{2}\right)\right)$, which is invariant under translations on $L$ (Spiegler and Jirsa, 2013). Each open surface, $L_{1}$ and $L_{2}$, is divided into $m=42$ areas, that is, $L_{1}=\cup_{r \in R 1}$ $A_{r}$ and $L_{2}=\cup_{r \in R_{2}} A_{r}$ with $R_{1}=R(m), R_{2}=R_{1}+n: R(\lambda \in \mathbb{N})=\{r \mid r \in \mathbb{N}, r \leq \lambda\}$, where $n=$ 428 is the number of subcortical areas. The division of the surfaces into areas follows the classification of volumes with regards to the tracer studies provided by the ABA, $A_{r}=A(\mathrm{r} \in$ $\mathbb{N}) \in \Omega: \mathbb{N} \rightarrow \mathbb{R}^{3}$ onto space $\Omega$ for introducing heterogeneous SC. Each of the $n=428$ considered subisocortical areas is lumped to a single point in space $S=\cup_{r_{\in} R_{3}} A_{r}$ with $R_{3}=$ $R(n)+2 m$. The heterogeneous connections, $C$ transmit mean activities of sources to target areas, $H(x)$ and $K\left(X^{\prime}\right)$ with a finite transmission speed, $v=1 \mathrm{~ms}^{-1}$. The square matrix, $C(\|$ $\left.x-X^{\prime} \| / v\right)$ contains $(2 m+n)^{2}$ weights, $c_{i j}\left(\left\|x-X^{\prime}\right\| / v\right): i, j=1, \ldots, 2 m+n$ taken from the ABA. The row vectors $H(x)$ and $K\left(X^{\prime}\right)$ contain $2 m+n$ operations, $h_{i}(x)$ and $k_{j}\left(X^{\prime}\right)$ on 
1126 the targets and sources, respectively. The operations are $h_{i}(x)=\delta_{x}\left(A_{i}\right)$ and $k_{j}\left(X^{\prime}\right)=\delta_{X^{\prime}}\left(A_{i}\right) /$

$1127\left|A_{j}\right|$ with the Dirac measure $\delta_{\Omega}(A)$ on $\Omega$ and the cardinality $\left|A_{r}\right|$ of the set $A_{r}$.

1128 The description of the large-scale brain network model (Equation 1) is fully compatible with 1129 previous TVB descriptions (Sanz-Leon et al., 2015; Spiegler et al., 2013, 2016). Note that the 1130 set notation is used here to describe brain areas and the division of homogeneously distributed

1131 and connected network nodes on both isocortices into cerebral areas.

\section{Local dynamics}

1133 The vector field describes a two-dimensional flow (Stefanescu and Jirsa, 2008) linking two

1134 variables $\Psi(x, t)=\left(\begin{array}{ll}\psi_{1} & \psi_{2}\end{array}\right)^{\mathrm{T}}(x, t)$ in $(1)$ as follows

$$
E(\Psi(x, t))=\eta\left(\begin{array}{c}
\psi_{2}(x, t)-\gamma \psi_{1}(x, t)-\psi_{1}^{3}(x, t) \\
-\varepsilon \psi_{1}(x, t)
\end{array}\right) .
$$

1136 The parameterization: $\gamma=1.21$ and $\varepsilon=12.3083$ sets an isolated brain area close to a critical

1137 point, that is, an Andronov-Hopf bifurcation (sketched in Figure 2) with a natural frequency 1138 around $42 \mathrm{~Hz}$ using a characteristic rate of $\eta=76.74 \mathrm{~s}^{-1}$. This rhythm in the gamma band 1139 accounts for local activity such as a coordinated interaction of excitation and inhibition 1140 (Buzsáki and Wang, 2012) that is not explicitly modeled here but represented by the surrogate 1141 parameter $\gamma$. The Dirac delta function is applied to a brain area, $I_{r}(x, t)=-5 \eta \delta_{x}\left(A_{r}\right) \delta(t)$. The 1142 connectivities and the input act on the first variable $\psi_{1}(x, t)$ in (1) by $a_{L}=a_{S}=\left(a_{I}\right)^{\mathrm{T}}=\left(\begin{array}{ll}\eta & 0\end{array}\right)$.

1143 The connectivity-weighted input determines criticality by working against inherent energy 1144 dissipation (i.e., stable focus) towards the bifurcation. So that the bifurcation was not passed, 1145 both homogeneous and heterogeneous SC, $g(x)$ and $C\left(\left\|\mathrm{x}-X^{\prime}\right\| / v\right)$ are normalized to unity maximum in-strength across time delays by: (i) $\int \mathrm{d} x$ $(x)=1$, and $\sup _{\lambda \in \Omega / v}\left\{\sum_{j}^{n} c_{i j}(\|\lambda\|)\right\}=1$.

\section{Simulation}

1149 To simulate the model on a digital computer, physical space and time are discretized. The 1150 folding of the human cortex presents a challenge for sampling. The surface of the left isocortex $L_{1}$ is evenly filled with 7,020 nodes and the surface for the right isocortex $L_{2}$ is evenly filled with 6,952 nodes. Subcortical structures in $S$ remain unaffected by the discretization. The geometry of the brain is captured in physical space, $\Omega$ by a net of 14,000 nodes (i.e. 13,972 cortical and 426 subisocortical nodes). The spatial integrals in (1) are rewritten as matrix operations, where the heterogeneous SC remains the same and the 
1156 homogeneous SC is spatially sampled on the cerebral surfaces (Spiegler and Jirsa, 2013). The 1157 system of difference equations are then solved using Heun's method with a time step of $40 \mu \mathrm{s}$ 1158 for 1 second per realization of one of the following factors: each of the 512 stimulation sites,

1159 SC-balance, $\alpha=\{0.0,0.2,0.4,0.6,0.8,1.0\}$, and homogeneous spreading, $\sigma / \mu \mathrm{m} \in \mathbb{N}: 500 \leq$ $1160 \sigma / \mu \mathrm{m} \leq 1000$. The implementation is verified by the algebraic solution of an isolated node 1161 (i.e., no connections), and by the field properties (e.g., compact solutions spreading radially 1162 around a stimulation site) of the homogeneously linked cerebral nodes.

1163 The lower bound of the spatial range of $\sigma=500 \mu \mathrm{m}$ results from the used geometrical model 1164 for the isocortex. A nearly regular mesh of triangles approximates each cerebral hemisphere 1165 with a finite edge length of $125 \mu \mathrm{m}$ on average (see Figure 11). The used Gaussian kernel for 1166 the homogeneous SC is sampled in the model through the cortical mesh. Because of the finite 1167 edge lengths in the mesh, the spatial range of the homogeneous SC should not fall below $1168207.78 \mu \mathrm{m}$ for $-3 \mathrm{~dB}$ cutoff of spatial frequencies with respect to their magnitude (Spiegler 1169 and Jirsa, 2013). The lower bound of the spatial range of $\sigma=500 \mu \mathrm{m}$ for the homogeneous 1170 Gaussian connectivity kernel causes a loss of at least $4.55 \%$ of spatial information (mainly 1171 short-range), which corresponds to $-17.37178 \mathrm{~dB}$ cutoff (see Spiegler and Jirsa, 2013).

\section{Stimulation and Decomposition}

1173 All network nodes of a brain area are constantly stimulated for a period of the characteristic 1174 time of the nodes, $\eta^{-1}$ to evoke damped oscillations with a maximum magnitude of one. The 1175 stimulation response of an isolated node is subtracted from the response of stimulated nodes 1176 in the network. A Principal Component Analysis (PCA) was performed using the covariance 1177 matrix among the 16,500 nodes. The period of $0.5 \mathrm{~s}$ data after $0.2 \mathrm{~s}$ of stimulus onset was 1178 decomposed (similar to Spiegler et al., 2016). For further analysis, up to three principal 1179 components (i.e., orthogonal) are considered that cover more than $99 \%$ of variance across 1180 conditions.

\section{Extracting dynamically responsive networks (DRNs)}

1182 The dot product of the normalized eigenvectors from the decomposition the stimulation 1183 response was used to measure the similarity of the dissipation across different stimulation 1184 sites for a range of values of the balance of the SC and a spatial range of the homogeneous 1185 SC. The eigenspaces are clustered based on the similarity measure using k-means for each 1186 SC-balance and each range of the homogeneous SC. The number of clusters is estimated via 1187 the gap statistic (Tibshirani et al., 2001). For each cluster, the eigenspaces are rotated to the 1188 basis of the one with the highest similarity among all in the cluster, using the singular value 
1189 decomposition and calculating the optimal rotation matrix (Kabsch, 1978). Averaging the 1190 aligned basis vectors in a cluster (across eigenspaces) gives the set of eigenvectors for each 1191 cluster. Each resulting eigenvector indicates the contribution of each network node (e.g., 1192 belonging to a cortical or a subcortical structure) to a DRN.

1193 Statistics on the DRNs and Prediction of the DRNs by the structural connectivity

1194 To performed statistics on the catalogue of DRNs we pair-wise correlated each of the three 1195 parts of each of the 24 motifs, that is, twelve motifs by stimulation of left brain areas and 1196 twelve motifs by stimulation of right areas (makes a total of 72 parts composing the 24 1197 motifs). The significance level of $1 \%$ was Bonferroni corrected by the total number of 1198 components. In addition, three thresholds are applied to the correlation values $C$ in order to 1199 find strong $(C>0.8)$, good $(0.6<C \leq 0.8)$, and moderate agreements $(0.5<C \leq 0.6)$ among 1200 the 72 parts of the 24 motifs. The analysis reveals a total of five significantly distinct spatial 1201 patterns in the motifs of the DRNs, indicating their entanglements.

1202 To test to which extent the topology in the structural connectivity predicts the brain activity 1203 due to stimulation, we calculated the following graph-theoretic measures on the long-range 1204 SC: in-, out-, total-degree; in-, out-, total-strength; and clustering coefficient (Rubinov and 1205 Sporns, 2010). Incoming, outgoing, or all connected ties to an area are measured in terms of 1206 (i) their numbers, and (ii) their weights. By counting the connections we obtain the in-, the 1207 out-, and the total-degree. By calculating the sum of connection weights we obtain the in-, the 1208 out-, and the total-strength. The clustering coefficient measures the degree to which areas in a 1209 graph tend to group together. We correlated the activity at the network nodes after stimulation 1210 with the graph theoretical characterization of the network nodes. Furthermore, we correlated 1211 the activity at the network nodes after stimulation with the connectivity strengths of the direct 1212 afferent (incoming connection to an area) and efferent connections (outgoing connection from 1213 an area). Each nine measures of the brain areas in the heterogeneous SC are then compared 1214 with the parts of each DRN (i.e., the eigenvector). For the correlation we used the Pearson 1215 correlation, the rank correlation (Kendall's tau) and the Bhattacharyya coefficient. To test 1216 statistical significance, the same permutation test is used as for the comparison of the DRNs 1217 with the functional networks. We Bonferroni corrected the significance level of 5\%.

\section{Sensory pathways}

1219 If not explicitly cited, the main reference for the description of the sensory pathways of the 1220 mouse brain is the book authored by Watson, Paxinos, and Puelles (2011). The visual pathways run from the retina through the hypothalamus, midbrain, and thalamus to terminate 
1222 in the primary visual cortices. The retinal ganglion cells bilaterally connect to the 1223 hypothalamus and all of them project to the (sensory related) superior colliculus according to 1224 the ipsilateral visual hemifield. The (sensory related) superior colliculus projects to the to 1225 areas of the cerebral cortex that are involved in controlling eye movements through the 1226 pulvinar and lateral posterior nucleus of the thalamus (Stein and Meredith, 1991). Pulvinar 1227 nuclei are virtually nonexistent in the rat, and grouped as the lateral posterior-pulvinar 1228 complex with the lateral posterior thalamic nucleus due to its small size in cats. In humans it 1229 makes up roughly $40 \%$ of the thalamus making it the largest of its nuclei (LaBerge, 1999). 1230 Furthermore, the (sensory related) superior colliculus also receives activity from other sensory systems such as whisker movements (Stein and Meredith, 1991). The retinal ganglion cells 1232 have ramifications to contralateral structures in the midbrain, namely nucleus of the optic 1233 tract, olivary pretectal and posterior pretectal nucleus (Pak et al., 1987) and ramifications that 1234 run into the primary visual cortex through the thalamus, namely intergeniculate leaflet as well 1235 as ventral and dorsal parts of the lateral geniculate complex according to the ipsilateral visual 1236 hemifield. Intergeniculate leaflet of the lateral geniculate complex seems to play an important 1237 role in mediating phases of the circadian rhythms that are not involved with light, as well as 1238 phase shifts that are light-dependent (Harrington, 1997; Edelstein and Amir, 1999). The 1239 network with the specific visual hemifield connections is summarized in Figure 6, Panel A. 1240 The auditory pathways originate from the cochleae to the primary auditory cortices through 1241 the dorsal and ventral cochlear nucleus of the medulla, nucleus of the lateral lemniscus of the 1242 pons, inferior colliculus, and nucleus of the brachium of the inferior colliculus of the midbrain, and the medial geniculate complex of the thalamus. The specific contra- and ipsilateral connections are summarized in Figure 6, Panel B. The pathways from the facial whiskers to the barrel fields run trough the medulla via the trigeminal nerve, the pons, the midbrain, and the thalamus to terminate in the somatosensory areas of the isocortex. In the different brain structures, each individual whisker is represented in a discrete anatomical unit, 1248 that is, a barrel (see Figure 6, Panel C). The major facial whiskers are topographically organized in the parts of the medulla, the pons, the thalamus, and the isocortex. The somatotopy of the whisker follicles mainly comes to the primary and secondary somatosensory cortices (barrel cortex) via the trigeminal nerve nuclei through the principal sensory nucleus of the trigeminal of the pons and caudal part of the spinal nucleus of the 1253 trigeminal of the medulla (somatotopic arrangement of barrelets), and the ventral 1254 posteromedial nucleus of the thalamus (somatotopic arrangement of barreloids). Specifically, 1255 the trigeminal nerve fibers have ramifications to caudal, interpolar, and oral part of the spinal 
1256 nucleus of the trigeminal (medulla), to the principal sensory nucleus of the trigeminal of the 1257 pons, and to the trigeminal nucleus of the midbrain (Bosman et al., 2011). Whisker movements can activate the sensory related parts of the superior colliculus of the midbrain through the trigeminal nucleus (Matesz, 1981; Ndiaye et al., 2000). The superior colliculus

1260 has other parts related to the sensory processing such as the visual system and eye 1261 movements. The main sensory pathway is conveys somatotopic information from the 1262 whiskers to the barrel field of the primary somatosensory areas of the isocortex via the 1263 principal sensory nucleus of the trigeminal (pons), and the ventral posteromedial nucleus 1264 (thalamus). The other minor pathways are as follows: The principal sensory nucleus of the 1265 trigeminal (part of the pons) mainly projects to the dorsomedial part (and less to the head 1266 area) of the ventral posteromedial nucleus of the thalamus, and less to the posterior complex 1267 of the thalamus. Posterior complex of the thalamus receives connections from the ventral 1268 posteromedial nucleus, the interpolar and oral parts of the spinal nucleus of the trigeminal and 1269 sends input to the primary and secondary somatosensory cortices as well as the primary motor cortices (Bosman et al., 2011; Lane et al., 2008). The caudal part of the spinal nucleus of the trigeminal sends input to the ventral posteromedial nucleus and to the lateral dorsal nucleus, which send input to the somatosensory areas. The specific contra- and ipsilateral connections are summarized in Figure 6, Panel D. The pathways from the fore and hind limbs pass via

1274 dorsal root ganglion to the ipsilateral dorsal column nuclei of the medulla, comprising the 1275 cuneate nucleus and the gracile nucleus. The upper limb, and especially the forelimb, connects through the cuneate nucleus, whereas the lower limb, and especially the hind limb, connects through the gracile nucleus. The nuclei of the medulla connect via the medial lemniscus to the contralateral ventral posterolateral nucleus of the thalamus, which has ipsilateral ramifications into the primary somatosensory cortex (S1). The specific contra- and ipsilateral connections are summarized in Figure 6, Panel E.

1281 We have quantified the quality of the sensory pathways represented in the ABA connectome 1282 as follows: A particular set of anatomical connections (structural connectivity), which are 1283 involved in the processing of a particular brain function, is embedded in a large-scale brain network forming sub-networks. Large-scale brain network of directed connections, which weights are assumed to be positively-defined, that is, between network nodes $i$ and $j: c_{i j} \neq c_{j i}$ !, and $c_{i j} \geq 0$ (the first index is the target and the latter is the source). A particular network

1287 comprises a set $\mathbf{R}$ of edges aka connections $\mathbf{R} \subseteq \mathbf{r}_{n}$ between index vertices $u \in \mathbb{N}$ and $v \in \mathbb{N}$ aka nodes in physical space $\Omega, \mathbf{r}_{n}=\{v \rightarrow u\}$. The arrow in the notation for the $n \in \mathbb{N}$ connection $\mathbf{r}_{n}$ indicates a directed projection from vertex $v$ onto vertex $u$. The embedding of a 
given connection can be characterized by its particular in- and out-strength in a given largescale brain network or alternatively the maximum in- and output value:

$$
E\left(\mathbf{r}_{n}\right)=\left\{\begin{array}{lll}
\frac{c_{u v}}{\sum_{b} c_{u b}} & \text { or } \frac{c_{u v}}{\max _{b}\left(c_{u b}\right)} \text { for target } u \\
\frac{c_{u v}}{\sum_{a} c_{a v}} & \text { or } \frac{c_{u v}}{\max _{a}\left(c_{a v}\right)} \text { for source } v
\end{array},\right.
$$

1293

where the maximum income of a target $u$ is $\max _{b}\left(c_{u b}\right)$ and the maximum output of a source $v$ is $\max _{a}\left(c_{a v}\right)$. Using both graph-theoretic measures color the edges $\mathbf{r}_{n}$ with a positive scalar $c_{n}$

$$
c_{n}(\mathbf{r})=c_{a b}^{2} \times\left\{\begin{array}{cl}
\frac{1}{\sum_{a} c_{a v} \sum_{b} c_{u b}}<1 & \text { colored by in- and out-stenght measure } \\
\frac{1}{\max _{a}\left(c_{a v}\right) \max _{b}\left(c_{u b}\right)} \leq 1 & \text { colored by maximum input and output }
\end{array}\right.
$$

The presence of a subnetwork of interest that is defined by $N$ connections $\mathbf{R} \subseteq \mathbf{r}_{n}: n=1, \ldots$, $N$ in a large-scale network $\mathbf{U}: \mathbf{R} \subseteq \mathbf{U}$ can be quantified as follows:

$$
\mathbf{R} / \mathbf{U}=\frac{1}{N} \sum_{n=1}^{N} c_{n}(\mathbf{r}) .
$$

We have used both normalization types to quantify the embedding of sensory pathways.

The Pearson correlation and the Bhattacharyya coefficient (Bhattacharyya, 1946) is then used to estimate the amount of overlap (i.e., the square root of the inner product) between a functional network and a DRN, which elements are essentially indicated by an eigenvector (part of a motif). The square of each eigenvector element is taken and summed up within each area. The coarse-grained eigenvectors are normalized to unit length. A functional networks and each normalized coarse-grained part of a DRNs are compared. The $p$-values are corrected due to 27 independent multiple comparisons (nine functional networks with three eigenvectors per stimulation site) with a significance level of $5 \%$.

\section{Comparing voltage sensitive dye imaging and simulated data}

We preprocessed the VSD imaging data by calculating the ratio of the deviation of the optical signal from the baseline and baseline $\left(\Delta F / F_{0}\right)$ for each pixel separately. Then we converted this ratio to percentage by multiplying it by 100 . We defined the baseline as the pre-stimulus level of the optical signal for each pixel. Therefore, a signal level of $0.2 \%$ in a particular pixel means that the optical signal captured from that pixel is $0.2 \%$ of the baseline for that pixel. Then, we used the $\Delta F / F_{0}$ signal to identify the activated pixels at each time point after the 
1315 stimulation was applied. We defined the onset of activation for a pixel as the timestamp at 1316 which its activity surpasses the $20 \%$ of its peak activity. By calculating the onset of the

1317 activation for all the cortical regions in our imaging window, we obtained the temporal order 1318 of activation of these regions. Moreover, we calculated the maximum of the $\Delta F / F_{0}$ signal for 1319 each pixel around a temporal neighborhood of the stimulation time point and obtained the 1320 maximum intensity maps in the panels $\mathbf{C}$ in Figure 8 to Figure 10 . These maps ease the 1321 qualitative investigation of the overlap of activated regions in the simulated and empirical 1322 data.

\section{In-vivo VSDI and optogenetic stimulation in the mouse brain}

1324 To perform the VSD imaging experiments, animals were anesthetized with $0.5 \%$ isoflurane, and a large portion of the dorsal skull on the right hemisphere as well as the dura matter were removed. Then, the exposed brain was incubated for 60 - 90 minutes with the dye RH1692 solution prepared with the HEPES-buffered saline $(1 \mathrm{mg} / \mathrm{ml})$. After the unbounded dye solution was washed out from the brain, the stained brain surface was covered with the $1.5 \%$ agarose dissolved in HEPES-buffered saline and sealed with a glass coverslip. The VSD data was collected as 12-bit images using a CCD camera (1M60 Pantera, Dalsa, Waterloo, ON) and EPIX E4DB frame grabber with XCAP 3.1 imaging software (EPIX, Inc., Buffalo Grove IL). The focal plane was set to about $1 \mathrm{~mm}$ bellow the cortical surface in order to reduce the potential brain surface pulsation artifacts. VSD was excited with the red light emitted from a LED (Luxeon K2, $627 \mathrm{~nm}$ center) and filtered in the range of $(630 \pm 15) \mathrm{nm}$. VSD fluorescence was filtered using a 673-703 nm optical bandpass filter (Semrock, New York, NY) before being captured by the CCD camera.

To identify the coordinates of the sensory areas, periphery stimulation combined with VSD imaging was performed. The peripheral somatosensory, visual, barrel, and auditory stimulation were performed by delivering $1 \mathrm{~mA}$ electrical current to the limbs, delivering a short-duration pulse of a green light to the left eye, shaking the whiskers using a pizo-coupled arm, and playing a sound clique next to the left ear, respectively.

1342 The output of a diode pumped solid-state laser emitting $473 \mathrm{~nm}$ light (CNI Optoelectronics, 1343 Changchun, China), was used to stimulate ChR2-expressing neurons. The laser beam was 1344 directed to pre-defined coordinates on the cortical surface using custom software written in 1345 IGOR PRO (Portland, OR), which controlled galvanometer scan mirrors (Cambridge Tech, 1346 Lexington, MA), via analog output voltage from PCI-6110 DAQ (National Instruments, 1347 Austin, TX). Low amplitude and short duration single laser pulses were applied to the cortical 1348 surface to ensure sufficient activation, low laser stimulus artifact, and low photo-bleaching. 


\section{REFERENCES}

1349 http://connectivity.brain-map.org

1350 http://www.mouseimaging.ca/research/mouse_atlas.html

1351 www.MouseConnectome.org

1352 http://thevirtualbrain.org/

1353 Bartolomei F, Lagarde S, Wendling F, McGonigal A, Jirsa V, Guye M, Bénar C (2017)

1354 Defining epileptogenic networks: Contribution of SEEG and signal analysis. Epilepsia

1355 58:1131-47, https://doi.org/10.1111/epi.13791Braitenberg V, Schüz A (1991) Anatomy of the

1356 cortex: statistics and geometry. Berlin/Heidelberg: Springer. http://dx.doi.org/10.1007/978-3-

$1357 \quad \underline{662-02728-8}$

1358 Bhattacharyya A (1946) On a measure of divergence between two multinomial populations.

1359 Sankhya 7:401-6. http://www.jstor.org/stable/25047882

1360 Bosman LW, Houweling AR, Owens CB, Tanke N, Shevchouk OT, Rahmati N, Teunissen

1361 WH, Ju C, Gong W, Koekkoek SK, De Zeeuw CI (2011) Anatomical pathways involved in

1362

1363 generating and sensing rhythmic whisker movements. Front Integr Neurosci 5:53, https://doi.org/10.3389/fnint.2011.00053

1364 Braitenberg V, Schüz A (1998) Cortex: statistics and geometry of neuronal connectivity.

1365 Berlin/Heidelberg: Springer. http://dx.doi.org/10.1007/978-3-662-03733-1

1366 Breakspear M (2017) Dynamic models of large-scale brain activity. Nat Neurosci 20:340-52, 1367 https://doi.org/10.1038/nn.4497

1368 Buzsáki G, Anastassiou CA, Koch C (2012) The origin of extracellular fields and currents 1369 EEG, ECoG, LFP and spikes. Nat Rev Neurosci 13:407-20, https://doi.org/10.1038/nrn3241

1370 Buzsáki G, Wang XJ (2012) Mechanisms of gamma oscillations. Annu Rev Neurosci 35:2031371 25. http://dx.doi.org/10.1146/annurev-neuro-062111-150444

1372 Calvert GA, Spence C, Stein BE (2004) The Handbook of Multisensory Processes.

1373 Cambridge, MA: MIT Press, ISBN 9780262033213

1374 David O, Kiebel SJ, Harrison LM, Mattout J, Kilner JM, Friston KJ (2006) Dynamic causal

1375 modeling of evoked responses in EEG and MEG. Neuroimage 30:1255-72,

1376 https://doi.org/10.1016/j.neuroimage.2005.10.045

1377 Deco G, Jirsa VK, McIntosh AR (2011) Emerging concepts for the dynamical organization of

1378 resting-state activity in the brain. Nat Rev Neurosci 12:43-56,

1379 http://dx.doi.org/10.1038/nrn2961

1380 Deco G, Jirsa VK, McIntosh AR (2013) Resting brains never rest: computational insights into potential cognitive architectures. Trends Neurosci 36:268-74,

1383 Dehaene S, Meyniel F, Wacongne C, Wang L, Pallier C (2015) The Neural Representation of 1384 Sequences: From Transition Probabilities to Algebraic Patterns and Linguistic Trees. Neuron 1385 88:2-19, https://doi.org/10.1016/j.neuron.2015.09.019

1386 Denk W, Delaney KR, Gelperin A, Kleinfeld D, Strowbridge BW, Tank DW, Yuste R (1994)

1387 Anatomical and functional imaging of neurons using 2-photon laser scanning microscopy. J

1388 Neurosci Methods 54:151-62, https://doi.org/10.1016/0165-0270(94)90189-9

1389 Dorr AE, Lerch JP, Spring S, Kabani N, Henkelman RM (2008) High resolution three-

1390 dimensional brain atlas using an average magnetic resonance image of 40 adult $\mathrm{C} 57 \mathrm{Bl} / 6 \mathrm{~J}$ 
mice. Neuroimage 42(1):60-9, https://doi.org/10.1016/j.neuroimage.2008.03.037Fagerholm ED, Lorenz R, Scott G, Dinov M, Hellyer PJ, Mirzaei N, Leeson C, Carmichael DW, Sharp DJ, Shew WL, Leech R (2015) Cascades and Cognitive State: Focused Attention Incurs Subcritical Dynamics. J Neurosci 35:4626-34, https://doi.org/10.1523/JNEUROSCI.369414.2015

Edelstein K, Amir S (1999) The role of the intergeniculate leaflet in entrainment of circadian rhythms to a skeleton photoperiod. J Neurosci 19:372-80, http://www.ncbi.nlm.nih.gov/pubmed/9870966

Foran DR, Peterson AC (1992) Myelin acquisition in the central nervous system of the mouse revealed by an MBP-Lac Z transgene. J Neurosci 12:4890-7.

http://www.jneurosci.org/cgi/pmidlookup?view=long\&pmid=1281497

Friston KJ, Harrison L, Penny W (2003) Dynamic causal modelling. Neuroimage 19:1273302, https://doi.org/10.1016/S1053-8119(03)00202-7

Ghosh A, Rho Y, McIntosh AR, Kötter R, Jirsa VK (2008) Noise during rest enables the exploration of the brain's dynamic repertoire. PLoS Comput Biol 4:e1000196, http://dx.doi.org/10.1371/journal.pcbi.1000196

Haken H (1978) Synergetics: an introduction nonequilibrium phase transitions and selforganization in physics, chemistry and biology. Berlin/Heidelberg: Springer, http://dx.doi.org/10.1007/978-3-642-96469-5

Harrington ME (1997) The ventral lateral geniculate nucleus and the intergeniculate leaflet: interrelated structures in the visual and circadian systems. Neurosci Biobehav Rev 21:705-27, https://doi.org/10.1016/S0149-7634(96)00019-X

Huys R, Perdikis D, Jirsa VK (2014) Functional architectures and structured flows on manifolds: a dynamical framework for motor behavior. Psychol Rev 121:302-36, https://doi.org/10.1037/a0037014

Krakauer JW, Ghazanfar AA, Gomez-Marin A, MacIver MA, Poeppel D (2017) Neuroscience Needs Behavior: Correcting a Reductionist Bias. Neuron 93:480-90, https://doi.org/10.1016/j.neuron.2016.12.041

Jirsa and Kelso, 2000 Jirsa VK, Kelso JAS (2000) Spatiotemporal pattern formation in neural systems with heterogeneous connection topologies. Phys Rev E Stat Phys Plasmas Fluids Relat Interdiscip Topics 62:8462-5, http://dx.doi.org/10.1103/PhysRevE.62.8462

Jirsa VK, Stacey WC, Quilichini PP, Ivanov AI, Bernard C (2014) On the nature of seizure dynamics. Brain 137:2210-30, https://doi.org/10.1093/brain/awu133

KabschW(1978) A discussion of the solution for the best rotation to relate two sets of vectors. Acta Crystallogr A 34:827-8, http://dx.doi.org/10.1107/S0567739478001680

Kunze T, Hunold A, Haueisen J, Jirsa V, Spiegler A (2016) Transcranial direct current stimulation changes resting state functional connectivity: a large-scale brain network modeling study. Neuroimage 140:174-87, http://dx.doi.org/10.1016/j.neuroimage.2016.02.015

LaBerge, D. (1999). Attention pp. 44-98. In Cognitive science (Handbook of Perception and Cognition, Second Edition), Bly BM, Rumelhart DE. (edits). Academic Press ISBN 978-012-601730-4 p. 73

Lane RD, Pluto CP, Kenmuir CL, Chiaia NL, Mooney RD (2008) Does reorganization in the cuneate nucleus following neonatal forelimb amputation influence development of anomalous 
1435 circuits within the somatosensory cortex? J Neurophysiol 99:866-75,

1436 https://doi.org/10.1152/jn.00867.2007

1437 Marder E, Taylor AL (2011) Multiple models to capture the variability in biological neurons

1438 and networks. Nat Neurosci 14:133-8, https://doi.org/10.1038/nn.2735

1439 Marlinski V, Beloozerova IN (2014) Burst firing of neurons in the thalamic reticular nucleus

1440 during locomotion. J Neurophysiol 112:181-92, https://doi.org/10.1152/jn.00366.2013

1441 Matesz C (1981) Peripheral and central distribution of fibres of the mesencephalic trigeminal

1442 root in the rat. Neurosci Lett 27:13-7, https://doi.org/10.1016/0304-3940(81)90198-1

1443 Melozzi F, Woodman MM, Jirsa VK, Bernard C (2017) The Virtual Mouse Brain: A

1444 Computational Neuroinformatics Platform to Study Whole Mouse Brain Dynamics. eNeuro:4,

1445 https://doi.org/10.1523/ENEURO.0111-17.2017

1446 Michel CM, Koenig T (2018) EEG microstates as a tool for studying the temporal dynamics

1447 of whole-brain neuronal networks: A review. Neuroimage 180:577-593,

1448 https://doi.org/10.1016/j.neuroimage.2017.11.062

1449 Mohajerani MH, Chan AW, Mohsenvand M, LeDue J, Liu R, McVea DA, Boyd JD, Wang

1450 YT, Reimers M, Murphy TH (2013) Spontaneous cortical activity alternates between motifs

1451 defined by regional axonal projections. Nat Neurosci 16:1426-35.

1452 https://doi.org/10.1038/nn.3499

1453 Ndiaye A, Pinganaud G, VanderWerf F, Buisseret-Delmas C, Buisseret P (2000) Connections

1454 between the trigeminal mesencephalic nucleus and the superior colliculus in the rat. Neurosci

1455 Lett 294:17-20, https://doi.org/10.1016/S0304-3940(00)01519-6

1456 Niedermeyer E (1997) Alpha rhythms as physiological and abnormal phenomena. Int J

1457 Psychophysiol 26:31-49, https://doi.org/10.1016/S0167-8760(97)00754-X

1458 Oh SW, Harris JA, Ng L, Winslow B, Cain N, Mihalas S, Wang Q, Lau C, Kuan L, Henry

1459 AM, Mortrud MT, Ouellette B, Nguyen TN, Sorensen SA, Slaughterbeck CR, Wakeman W,

1460 Li Y, Feng D, Ho A, Nicholas E, Hirokawa KE, Bohn P, Joines KM, Peng H, Hawrylycz MJ,

1461 Phillips JW, Hohmann JG, Wohnoutka P, Gerfen CR, Koch C, Bernard A, Dang C, Jones AR,

1462 Zeng H (2014) A mesoscale connectome of the mouse brain. Nature 508:207-14.

1463 https://doi.org/10.1038/nature13186

1464 Otnes RK, Enochson L (1972) Digital time series analysis. ( $1^{\text {st }}$ edition) New York: Wiley.

1465 ISBN 0471657190

1466 Pak MW, Giolli RA, Pinto LH, Mangini NJ, Gregory KM, Vanable JW Jr. (1987)

1467 Retinopretectal and accessory optic projections of normal mice and the OKN-defective

1468 mutant mice beige, beige-J, and pearl. J Comp Neurol 258:435-46,

1469 https://doi.org/10.1002/cne.902580311

1470 Palmigiano A, Geisel T, Wolf F, Battaglia D (2017) Flexible information routing by transient

1471 synchrony. Nat Neurosci 20:1014-22, https://doi.org/10.1038/nn.4569

1472 Paxinos G, Franklin K (2001) The Mouse Brain in Stereotaxic Coordinates. (2 ${ }^{\text {nd }}$ edition) San

1473 Diego: Academic Press. ISBN 0125476361

1474 Pfurtscheller G, Lopes da Silva FH (2001) Event-related EEG/MEG synchronization and

1475 desynchronization: basic principles. Clin Neurophysiol 110:1842-57,

1476 https://doi.org/10.1016/S1388-2457(99)00141-8

1477 Pillai AS, Jirsa VK (2017) Symmetry Breaking in Space-Time Hierarchies Shapes Brain

1478 Dynamics and Behavior. Neuron 94:1010-26, https://doi.org/10.1016/j.neuron.2017.05.013 
1479 Proix T, Bartolomei F, Chauvel P, Bernard C, Jirsa VK (2014) Permittivity coupling across

1480 brain regions determines seizure recruitment in partial epilepsy. J Neurosci 34:15009-21, https://doi.org/10.1523/JNEUROSCI.1570-14.2014

1482 Proix T, Spiegler A, Schirner M, Rothmeier S, Ritter P, Jirsa VK (2016) How do parcellation size and short-range connectivity affect dynamics in large-scale brain network models? Neuroimage 142:135-149, https://doi.org/10.1016/j.neuroimage.2016.06.016

1485 Qubbaj MR, Jirsa VK (2007) Neural field dynamics with heterogeneous connection topology.

1486 Phys Rev Lett 98:238102, http://dx.doi.org/10.1103/PhysRevLett.98.238102

1487 Qubbaj MR, Jirsa VK (2009) Neural field dynamics under variation of local and global

1488 connectivity and finite transmission speed. Physica D 238:2331-46,

1489 http://dx.doi.org/10.1016/j.physd.2009.09.014

1490 Rabinovich M, Huerta R, Laurent G (2008) Neuroscience. Transient dynamics for neural processing. Science. 321:48-50, https://doi.org/10.1126/science.1155564

1492 Raue A, Kreutz C, Maiwald T, Bachmann J, Schilling M, Klingmüller U, Timmer J (2009)

1493 Structural and practical identifiability analysis of partially observed dynamical models by

1494 exploiting the profile likelihood. Bioinformatics 25:1923-9,

1495 https://doi.org/10.1093/bioinformatics/btp358

1496 Rosanova M, Casali A, Bellina V, Resta F, Mariotti M, Massimini M (2009) Natural

1497 frequencies of human corticothalamic circuits. J Neurosci 29:7679-85,

1498 https://doi.org/10.1523/JNEUROSCI.0445-09.2009

1499 Rubinov M, Sporns O (2010) Complex network measures of brain connectivity: uses and

1500 interpretations. Neuroimage 52:1059-69, http://dx.doi.org/10.1016/j.neuroimage.2009.10.003

1501 Salami M, Itami C, Tsumoto T, Kimura F (2003) Change of conduction velocity by regional

1502 myelination yields constant latency irrespective of distance between thalamus and cortex,

1503 Proc Natl Acad Sci USA, 100(10): 6174-9, http://dx.doi.org/10.1073/pnas.0937380100

1504 Sanz-Leon P, Knock SA, Woodman MM, Domide L, Mersmann J, McIntosh AR, Jirsa V (2013) The virtual brain: a simulator of primate brain network dynamics. Front Neuroinform 7:10, http://dx.doi.org/10.3389/fninf.2013.00010

1507 Sanz-Leon P, Knock SA, Spiegler A, Jirsa VK (2015) Mathematical framework for large1508 scale brain network modeling in the virtual brain. Neuroimage 111:385-430,

1509 http://dx.doi.org/10.1016/j.neuroimage.2015.01.002

1510 Schüz A, Chaimow D, Liewald D, Dortenman M (2006) Quantitative Aspects of

1511 Corticocortical Connections: A Tracer Study in the Mouse, Cereb Cortex 6(10):1474-86,

1512 https://doi.org/10.1093/cercor/bhj085

1513 Sforazzini F, Schwarz AJ, Galbusera A, Bifone A, Gozzi A (2014) Distributed BOLD and

1514 CBV-weighted resting-state networks in the mouse brain, NeuroImage, 87:403-15,

1515 https://doi.org/10.1016/j.neuroimage.2013.09.050

1516 Shah AS, Bressler SL, Knuth KH, Ding M, Mehta AD, Ulbert I, Schroeder CE (2004) Neural 1517 dynamics and the fundamental mechanisms of event-related brain potentials. Cereb Cortex 1518 14:476-83, https://doi.org/10.1093/cercor/bhh009

1519 Sherman SM (2001) Tonic and burst firing: dual modes of thalamocortical relay, Trends 1520 Neurosci 24:122-6, https://doi.org/10.1016/S0166-2236(00)01714-8

1521 Shew WL, Plenz D (2013) The functional benefits of criticality in the cortex. Neuroscientist 19:88-100, https://doi.org/10.1177/1073858412445487 
1523 Spiegler A, Jirsa V (2013) Systematic approximations of neural fields through networks of

1524 neural masses in the virtual brain. Neuroimage:83:704-25.

1525 https://doi.org/10.1016/j.neuroimage.2013.06.018

1526 Spiegler A, Hansen EC, Bernard C, McIntosh AR, Jirsa VK (2016) Selective Activation of

1527 Resting-State Networks following Focal Stimulation in a Connectome-Based Network Model

1528 of the Human Brain. eNeuro:3, https://doi.org/10.1523/ENEURO.0068-16.2016

1529 Stefanescu RA, Jirsa VK (2008) A low dimensional description of globally coupled

1530 heterogeneous neural networks of excitatory and inhibitory neurons. PLoS Comput Biol

1531 4:e1000219, http://dx.doi.org/10.1371/journal.pcbi.1000219

1532 Stein BE, and Meredith MA. Functional organization of the superior colliculus. In Leventhal

1533 AG ed. The neural basis of visual function, vol 4. Basingstoke: Macmillan; 1991.

1534 Svoboda K, Yasuda R (2006) Principles of two-photon excitation microscopy and its

1535 applications to neuroscience. Neuron 50:823-39,

1536 https://doi.org/10.1016/j.neuron.2006.05.019

1537 Swadlow HA, Waxman SG (2012) Axonal conduction delays, Scholarpedia, 7(6):1451,

1538 http://dx.doi.org/10.4249/scholarpedia.1451

1539 Tibshirani R, Walther G, Hastie T (2001) Estimating the number of clusters in a data set via

1540 the gap statistic. J R Stat Soc Series B Stat Methodol 63:411-23,

$1541 \quad$ http://dx.doi.org/10.1111/1467-9868.00293

1542 Van de Ville D, Britz J, Michel CM (2010) EEG microstate sequences in healthy humans at

1543 rest reveal scale-free dynamics. Proc Natl Acad Sci U S A 107:18179-84,

1544 https://doi.org/10.1073/pnas.1007841107

1545 Wang SS, Shultz JR, Burish MJ, Harrison KH, Hof PR, Towns LC, Wagers MW, Wyatt KD

1546 (2008) Functional Trade-Offs in White Matter Axonal Scaling, J Neurosci 28(15): 4047-56,

1547 https://doi.org/10.1523/JNEUROSCI.5559-05.2008

1548 Watson C, Paxinos G, and Puelles L (2011) The Mouse Nervous System. (1 ${ }^{\text {st }}$ edition) San

1549 Diego: Academic Press Inc. ISBN 9780123694973

1550 Welsh DK, Logothetis DE, Meister M, Reppert SM (1995) Individual neurons dissociated

1551 from rat suprachiasmatic nucleus express independently phased circadian firing rhythms.

1552 Neuron 14:697-706, https://doi.org/10.1016/0896-6273(95)90214-7

1553 Zingg B, Hintiryan H, Gou L, Song MY, Bay M, Bienkowski MS, Foster NN, Yamashita S,

1554 Bowman I, Toga AW, Dong HW (2016) Neural networks of the mouse neocortex. Cell

1555 156:1096-111, https://doi.org/10.1016/j.cell.2014.02.023 


\section{FIGURES}

A

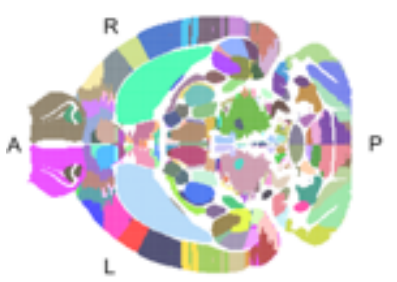

B

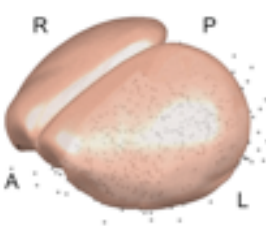

C

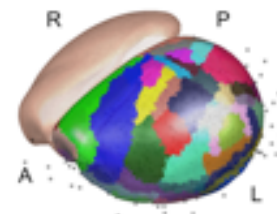

D

homogeneous connectivity

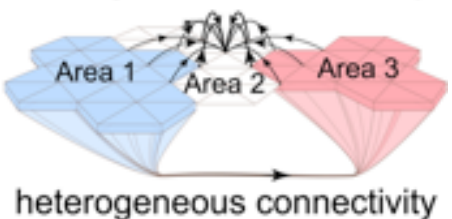

E

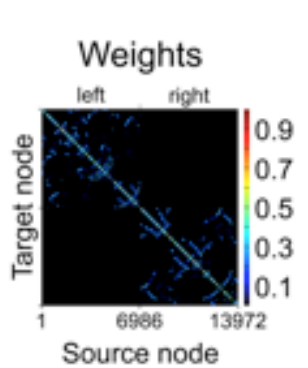

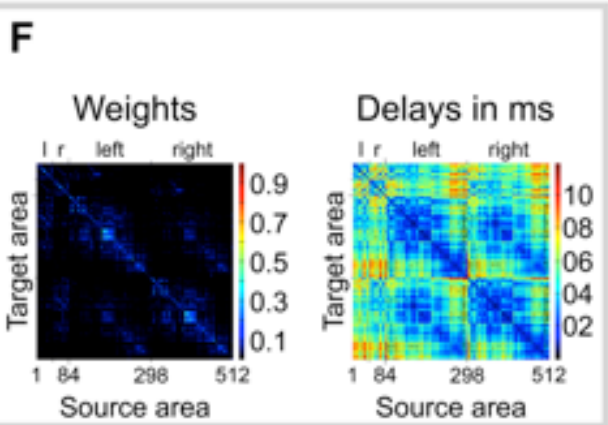

G
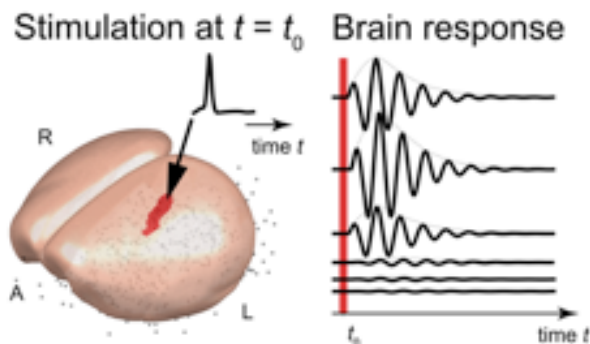

Figure 1. Modeling the mouse brain and simulating the mouse brain's response to focal

stimulation. The ABA database is isotropically sampled with a resolution of $50 \mu \mathrm{m}$ resulting in a total number of $3,300,706$ voxels $(1,653,785$ voxels for left side), which encode the injection by its spatial location and connectivity among 512 brain areas. The 512 brain areas are listed in Table 2 and Table 3. Panel A shows an axial slice of the sampled database. Each dot indicates the center of a voxel and the color indicates the different brain areas. To model large-scale activity of the cortex such as recorded using VSD imaging, the surface of the isocortex is reconstructed from the ABA database (slice shown in panel A) using a regular triangle mesh of 7,020 vertices for the left and 6,952 vertices for the right hemisphere. The quality of the mesh is quantified in Figure 11. Panel B shows the reconstructed isocortical surfaces. All other areas are lumped in their spatial extent (in the ABA) to point masses (centroids) indicated by the grey circles in panels $\mathbf{B}, \mathbf{C}$, and $\mathbf{G}$. Panel $\mathbf{C}$ : each vertex in the isocortical meshes is assigned to an area by the five closest voxels to the normal vector extended inside the volume of the ABA (slice shown in panel $\mathbf{A}$ ). The areas of the isocortex and the number of vertices per area are listed in Table 1. Panel D: The model distinguished two types of structural connectivity. Heterogeneous SC and corresponds to the connections extracted from the ABA such as tracts connecting brain areas over long distances. Homogeneous SC corresponds to gray matter fibers in the isocortex, with short-range connections within a given isocortical area, but also enabling some communication over short distances between neighboring areas. Although in the ABA, cortical area 2 is not connected to areas 1 and 3 , it is weakly linked to both areas via a set of short-range SC. Panel E: 
1578 color-coded. The diagonal describes in warm colors the strong SC of adjacent nodes. SC 1579 decreases with distance, which is shown in cold colors. SC of nearby nodes are scattered (e.g., 1580 blue dots) in panel $\mathbf{E}$ because each cerebral hemisphere is described by a surface, which 1581 makes it impossible to cluster nodes locally along both axes. Note the absence on 1582 interhemispheric short-range SC. Panel F: Heterogeneous SC for the 512 (84 isocortical plus 1583428 subcortical) brain areas for weights (left) and time delays (right). Within one hemisphere, 1584 the 214 sub-isocortical areas mostly project to the 42 isocortical areas. Some connections 1585 between subcortical areas can also be seen. The 42 cortical areas project heavily to both cortical and subcortical areas. Some interhemispheric connections can also been seen. Note 1587 also the presence of relatively large time delays. Panel G: The stimulation paradigm. The 1588 dynamics are simulated without noise. For that reason the activity is flat before stimulation. 1589 The brief stimulation causes activity in the targeted brain area, in panel $\mathbf{G}$ it is the unassigned 1590 left primary somatosensory area, and in the entire mouse brain model. The spread and the 1591 dissipation of the activity caused by the stimulation depend on the SC and the transmission 1592 delays. Each of the 512 cortical and subcortical brain areas is stimulated and the set of characteristic spatial activations of the brain response is extracted and investigated. 
a
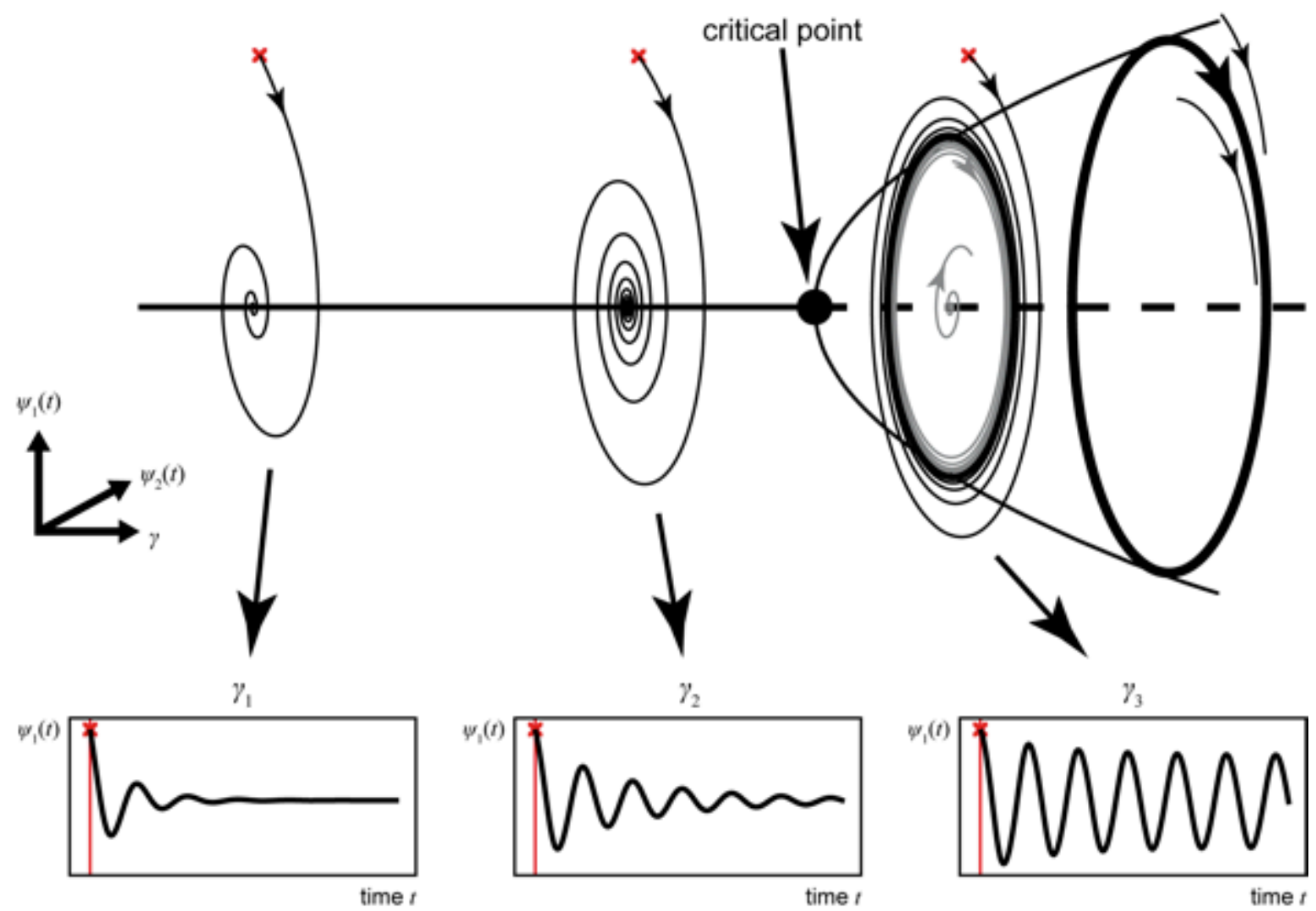

b

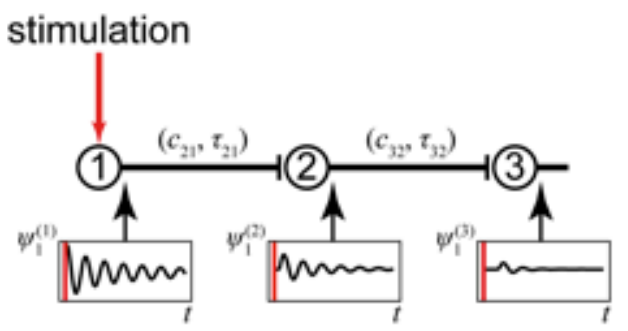

C

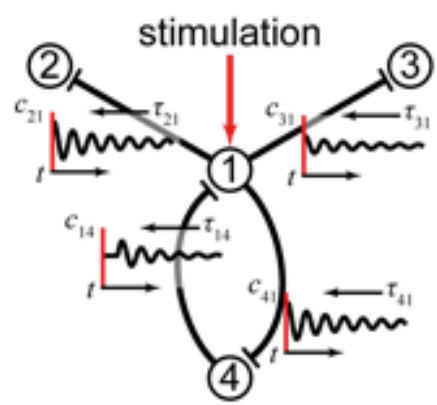

d

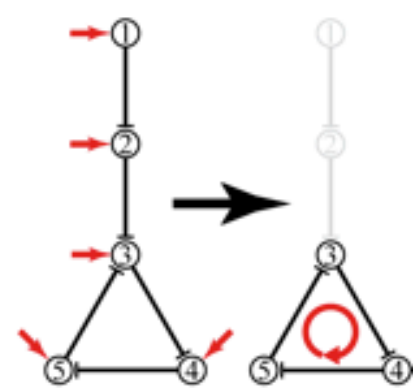

1594 Figure 2. The large-scale brain model works near criticality. Panel A: Each node in the model

1595 is parameterized by $\gamma$ to operate intrinsically at the same distance from the critical point if

1596 unconnected. A node shows zero activity or oscillation $(42 \mathrm{~Hz})$ in response to stimulation (red

1597 crosses). The activity at each node is described by two time-dependent variables, $\psi_{1}(t)$ and $\psi_{2}(\mathrm{t})$. The closer a node operates to the critical point, the larger and the longer lasting is the oscillation (compare $\gamma_{1}$ and $\gamma_{2}$ ). When the critical point is reached, the node intrinsically performs a rhythm of constant magnitude. The model, however, is set so that the critical point is never exceeded. Panel B: Principles of activity spreading after stimulation. The damped oscillation generated in the stimulated node (1) is sent via its efferent connections to its target node (2), triggering there, in turn, a damped oscillation with weaker amplitude and faster decay, which then propagates to the next node. Activity $\psi^{(j)}{ }_{1}(t)$ of node $(j)$ is scaled by $c_{i j}$ transmitted to node $(i)$ via homogeneous and heterogeneous connections (SCs), delayed by $\tau_{i j}$ 
1606 in the latter case. In such a chain, activity would decay fast. Panel C: In the large-scale brain 1607 model, multiple activity re-entry points can be found. At any time point, the dynamics of a 1608 node is influenced by all incoming activity. The response of the node to stimulation (1) is 1609 relayed to linked nodes (2-4), which may be fed back to 1 via 4 and may allow the induced 1610 activity to dissipate on a much longer time scale. The network response thus depends upon 1611 the SC and allows the network to operate near criticality. Panel D: Activation of dynamically 1612 responsive networks. Activity after stimulating a node (1 or 2$)$ in a series connection decays 1613 fast (as in panel B). However, activity may circulate and thus decays slower in a feedback 1614 network (of nodes 3-5). Such remaining activity after the initial stimulation decay reveals the so-called dynamically responsive networks (DRNs).

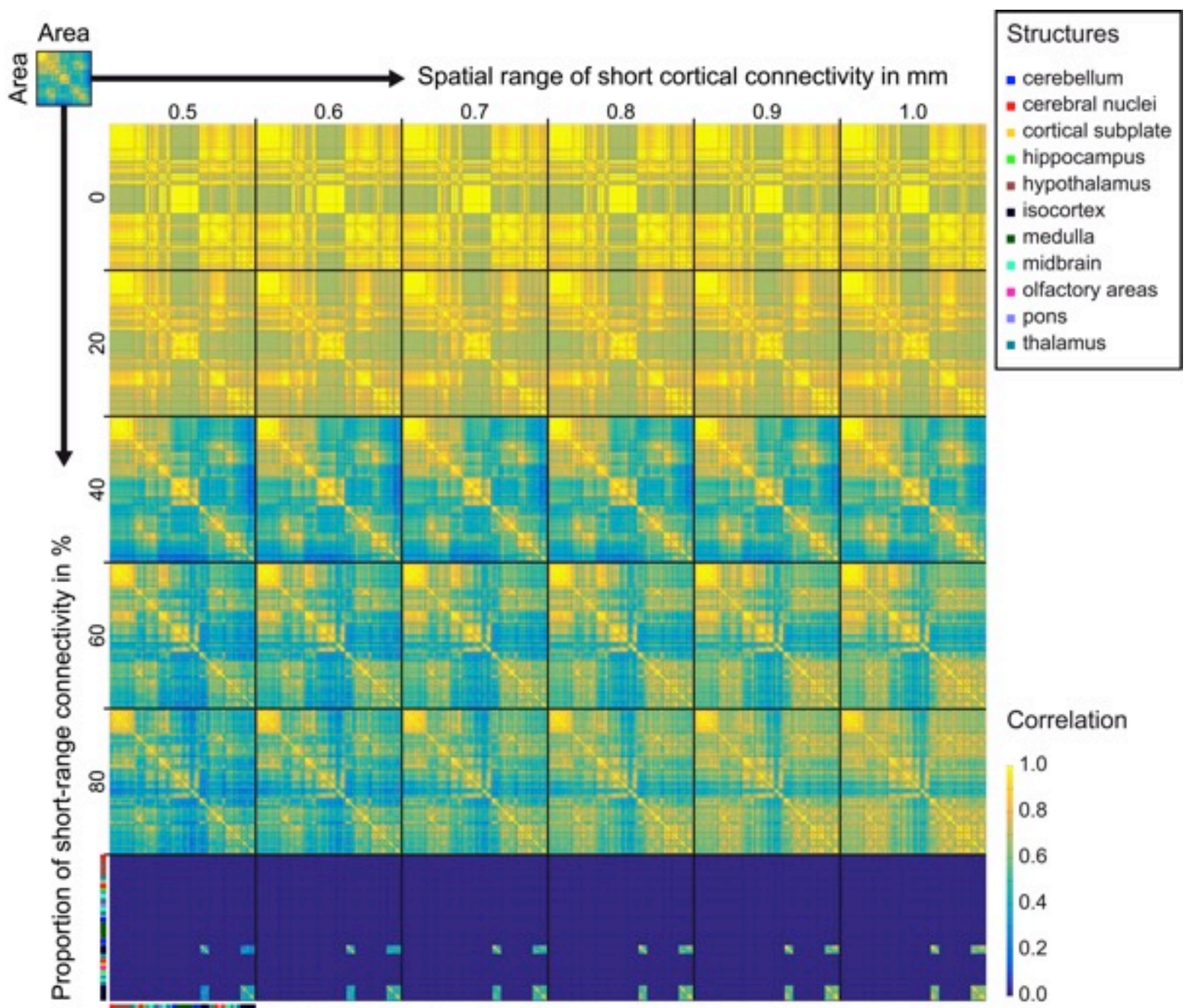

1616 Figure 3. Similarity matrices of the dynamically responsive networks (DRNs) between

1617 stimulated brain areas reveal a consistent formation of spatially similar brain activity in the 1618 mouse as a function of the proportion of short- and long-range connectivities in the 1619 communication between brain areas and the length of short-range connections. Structural 1620 connectivity forms clusters of functional networks that are responsive to sets of different 
1621 stimulation sites. This is summarized in a similarity matrix for a given proportion of

1622 homogeneous short-range to heterogeneous long-range connectivities in the model (varied 1623 along rows) and for a given length of short-range connections (varied along columns). The 1624 similarity of DRNs increases with warmer colors (yellow indicates maximum similarity). All 1625 similarity matrices are equally sorted and the color bar presented on the axes of the matrix for 1626 a spatial range of $500 \mu \mathrm{m}$ and $100 \%$ of short-range homogeneous connectivity (i.e., no long1627 range heterogeneous connectivity) indicates the brain structures (e.g., cerebral nuclei) to 1628 which a brain area is part of (e.g., nucleus accumbens and fundus of striatum are cerebral nuclei in the mouse model). The results indicate that long-range heterogeneous and shortrange homogeneous connections in the mouse shape functional networks but changes in the structural connectivities not necessarily cause reorganization of networks. The similarity matrices of the DRNs to stimulation were almost unchanged with changes in the length of short-range connections (see the similarity matrices along the columns). The short-range 1634 heterogeneous connectivity (see the row of $0 \%$ short-range homogeneous connectivity) supported DRNs that differ from the DRNs supported the short-range homogeneous connectivity (see the row of $100 \%$ short-range homogeneous connectivity). Both similarity matrices merge in the mix of short- and long-range connections (see the similarity matrices 1638 along the rows).

A

n cerebellum
n cerebral nuclei
n cortical subplate
nippocampus
n hypothalamus
n isocortex
n medulla
midbrain
molfactory areas
n pons
n thalamus

1639

1640

1641

1642

1643

1644

1645

1646
B

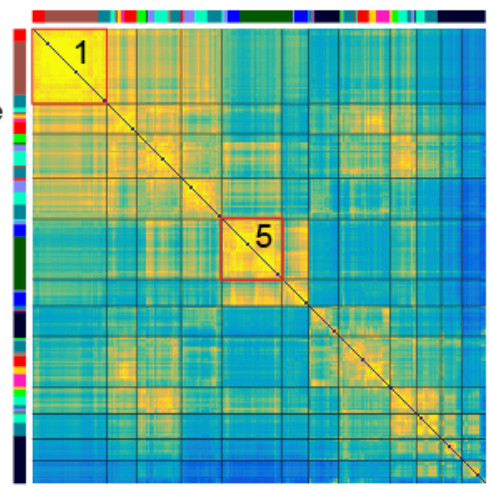

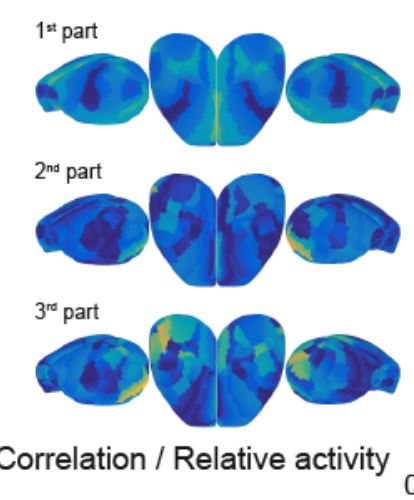

C

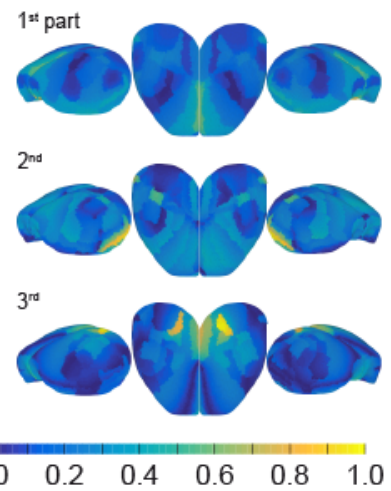

Figure 4. Specific focal stimulations activate similar networks. Panel $\mathbf{A}$ is the similarity matrix of the dynamically responsive networks (DRNs) for stimulation of the 512 different subareas. The stimulation sites and the DRNs form 12 clusters (separated by the black lines). This suggests that similar functional networks are activated in the mouse brain in response to stimulation of the different sites within a cluster. The color bar on the $x$ - and on the $y$-axis indicates the structures to which a brain area is part of. The similarity matrix in panel $\mathbf{A}$ is for a spatial range of $500 \mu \mathrm{m}$ and $40 \%$ of short-range homogeneous connectivity (i.e., $60 \%$ of long-range heterogeneous connectivity). Similarity of DRNs is color coded (warmer color 
1647 means more similarity). Panels $\mathbf{B}$ and $\mathbf{C}$ show the spatial organization of brain activity in 1648 the isocortex that corresponds to the clusters 1 and 5 as an example from the left, top, and 1649 right view. Three parts compose the motif of each DRN. The blue to yellow scale gives the 1650 relative contribution of areas to the response. The complete set of motifs, that is, the averaged 1651 brain activity of DRNs in each of the 12 clusters in panel A (numbered up from top to 1652 bottom) is shown in Figure 5.

A
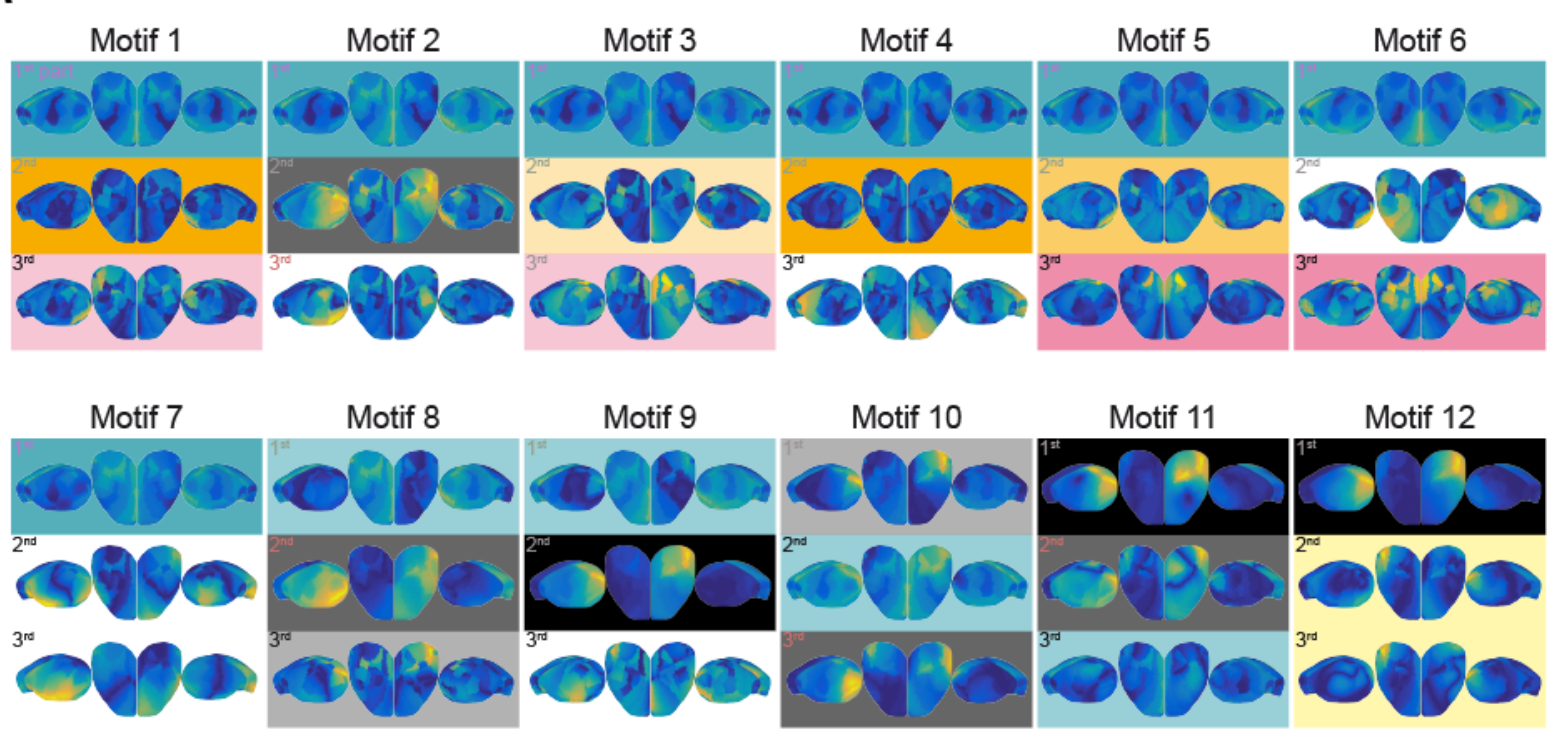

B
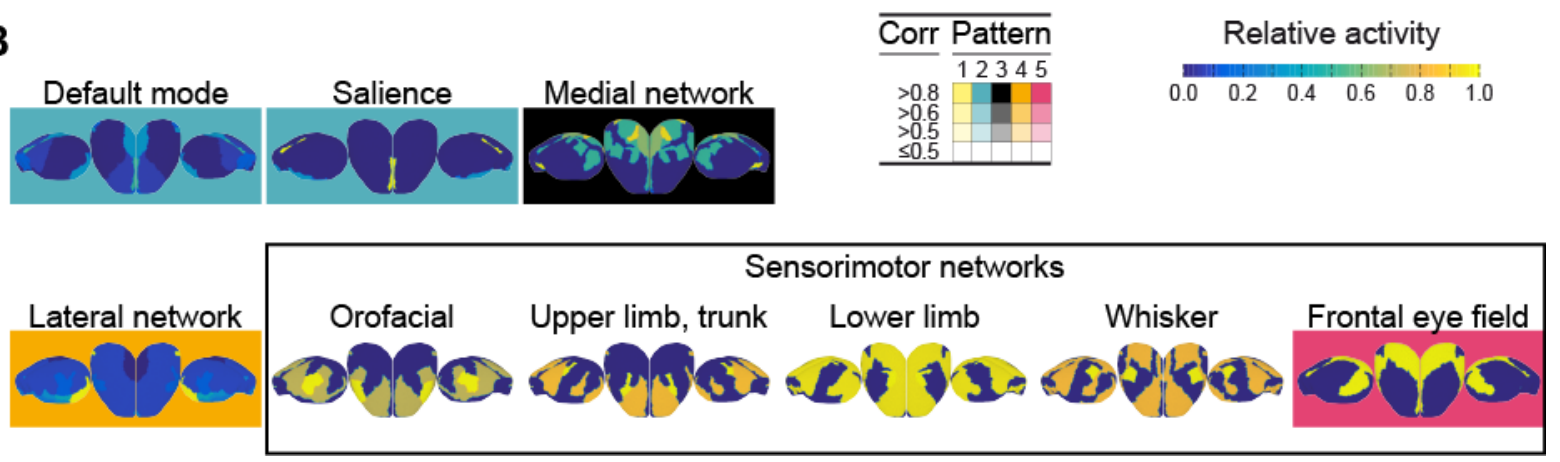

1653 Figure 5. Dynamically responsive networks (DRNs) in the mouse brain model. Panel A: The

1654 responses of the mouse brain to focal stimulation dissipate in 12 motifs, that is, the DRNs.

1655 Each motif proceeds from similar network activity after systematic stimulation of different

1656 sites (see Figure 4). Each dynamically responsive network is composed of three components

1657 (parts) covering 99\% of the activity induced by the stimulation. Each motif, in panel A, shows

1658 the averaged spatial activity (topography) for each part (1 to 3) across the similar network 1659 responses for the isocortices from the left, top, and right view. Similar parts among the motifs 1660 are highlighted. The five distinct patterns in the parts entangle the different motifs. The 1661 different shade indicates the correlation level (Corr) of the components (no shade, that is, 1662 white for not significant correlations and shades: dark, medium, light for significant 
1663 correlation values greater than $0.8,0.6,0.5)$. Six components are unique (i.e., parts in motifs $16642,4,6,7$, and 9) and not correlated to other motif parts. This makes motif 7 the most unique 1665 one and motif 1 the most entangled motif (followed by the entanglements in motifs 5, 11, and 1666 12). In order to interpret the DRNs, the components were correlated to functional networks. 1667 Panel B: Experimentally known resting state and functional networks (see description in the 1668 Materials and Methods) were compared to each part of each motif. The organization of brain 1669 activity in the isocortex is shown in Panel $\mathbf{B}$ for each of the nine functional networks from the 1670 left, top, and right view. Four experimentally known functional networks correlate with the 1671 patterns in the parts of the motifs and are highlighted in accordance with Panel $\mathbf{A}$. Note that 1672 the motifs do not cover the isocortical organization of sensory motor networks (neither 1673 left/right symmetric nor lateral) except for the frontal eye field network. The activity of the 1674 frontal eye field network within one hemisphere (lateralized organization) corresponds to 1675 pattern 4, which is highlighted in magenta. The unique parts in motifs 2, 4, 6, 7, and 9 (see 1676 parts in Panel A that are not highlighted) are not correlated with one of the nine specific 1677 functional networks. Moreover, pattern 1 (highlighted in yellow) in motif 12 does not correspond with any of the nine functional networks. The default mode and the salience networks show similarities at the isocortical level. Both comprised most of the motifs (cyan pattern 2 in motifs 1 to 11). Stimulation responses do activate to a lesser extent the lateral

1681 network (orange pattern 4 in motifs 1, and 3 to 5) and the medial network (gray pattern 3 in 1682 motifs 8 to 12), where the activity of both networks within one hemisphere (lateralized 1683 organization) is reflected. The patterns ( 1 to 5$)$ in the parts link the motifs (1 to 12). The 1684 default mode network (and the salience network) is thus related in the model to the most flexible and sensitive pattern (cyan pattern number 2) that can activate and involve most of the motifs (1 to 11). In both panels, $\mathbf{A}$ and $\mathbf{B}$, the blue to yellow scale gives the relative contribution of isocortical areas to the response. The order of the clusters is consistent with Figure 4 from top to bottom. 
A

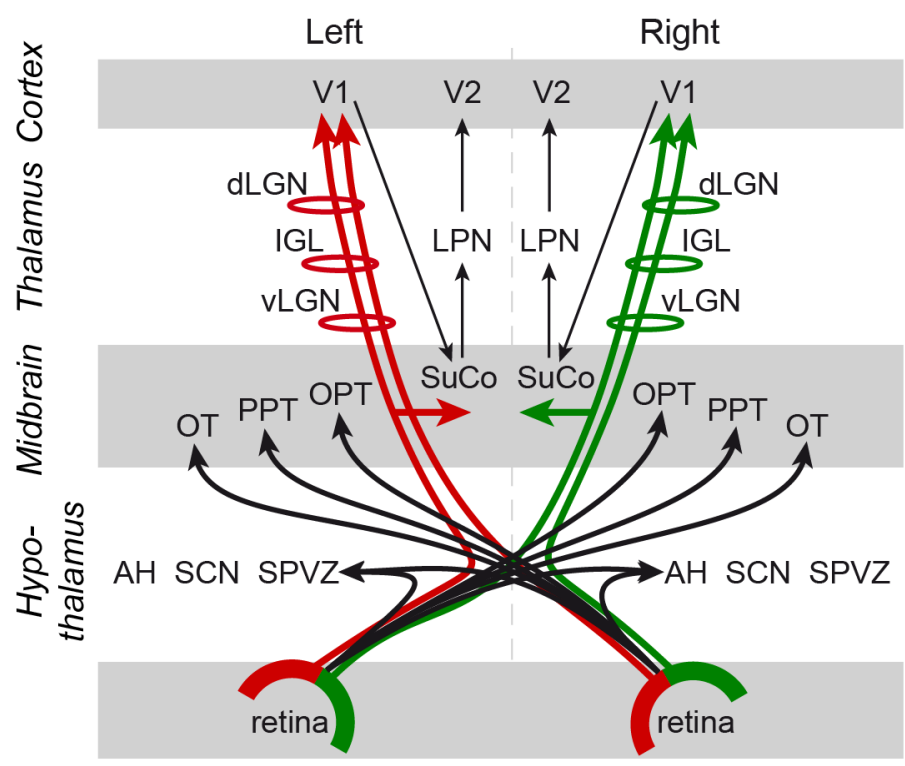

C

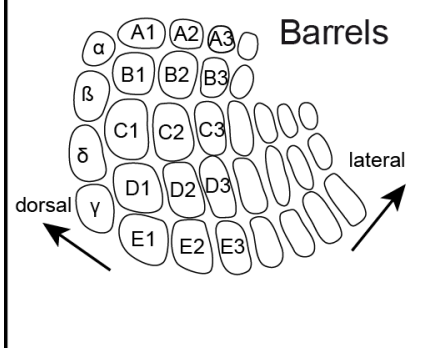

Whiskers 1234

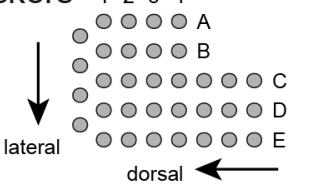

D

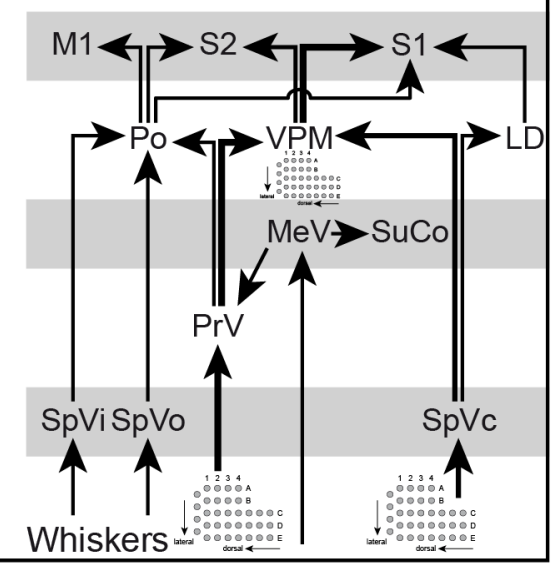

B

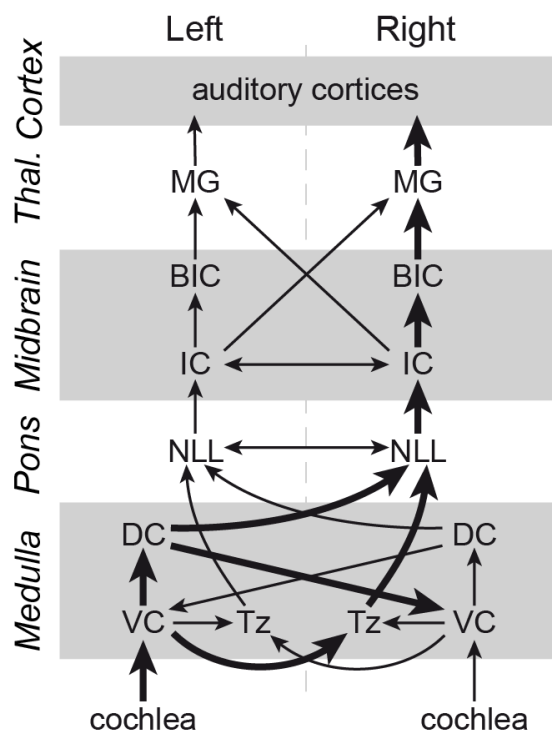

E

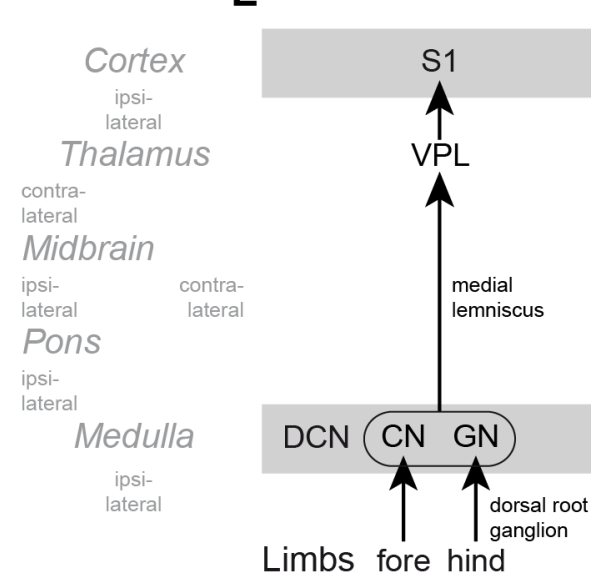

Figure 6. Networks of sensory pathways. Panel A: The visual pathways run from the retina through the hypothalamus, midbrain, and thalamus to terminate in the primary visual cortices.

1691 The retinal ganglion cells bilaterally connect to the hypothalamus and all of them project to

1692 the SuCo according to the ipsilateral visual field (colored links). The SuCo projects to the to 1693 areas of the cerebral cortex that are involved in controlling eye movements through the LPN 1694 of the thalamus. Note that the SuCo also receives activity due to whisker movements. The 1695 retinal ganglion cells have ramifications to contralateral structures in the midbrain (OT, PPT, 1696 OPT) and ramifications that run into the primary visual cortex through the thalamus (vLGN, 1697 IGL, dLGN) according to the ipsilateral visual field. The projections from the visuals fields 1698 through the retinal ganglion cells are highlighted in panel $\mathbf{A}$ by red and green colored links. 1699 The arrowheads indicate the termination areas of the axonal fibers. Panel B: The auditory 1700 pathways originate from the cochleae through the medulla, pons, midbrain, and thalamus to 1701 the primary auditory cortices. The thickness of the links in panel $\mathbf{B}$ indicates the strength of 1702 connectivity. Panels $\mathbf{C}-\mathbf{D}$ : The pathways from the whiskers to the barrel fields run trough the 
1703 medulla, pons, midbrain, and thalamus to terminate in the isocortex. Panel C: The 1704 somatotopic map of the whiskers where each individual whisker is represented in a discrete anatomical unit, that is, a barrel. The map shows the correspondence of the topographical organization of the major facial whiskers through the trigeminal nerve in the nuclei in the medulla ( $\mathrm{SpVc}$ ), the pons (barrelets), and through the thalamus (barreloids in VPM) to the somatosensory cortices (barrel cortex). Panel D: The pathways from the facial whiskers to the isocortex. The somatotopy of the whisker follicles mainly comes to the somatosensory cortices (i.e., S1, S2) via the trigeminal nerve nuclei ( $\mathrm{PrV}$ in the pons and $\mathrm{SpVc}$ in the medulla) and the thalamus (VPM). The thickness of the links in panel $\mathbf{D}$ indicates the strength of connectivity. Whisker movements activate the SuCo in the midbrain through the $\mathrm{MeV}$. The SuCo is also related to the visual system (see panel $\mathbf{A}$ ) and in particular to eye movements. Panel E: The pathways from the fore and hind limbs pass via dorsal root ganglion to the ipsilateral dorsal column nuclei (DCN) in the medulla (CN and GN). The upper limb, and especially the forelimb, connects through the $\mathrm{CN}$ whereas the lower limb, and especially the hind limb, connects through the GN in the medulla. The nuclei of the medulla connect via the medial lemniscus to the contralateral thalamus (VPL), which has ipsilateral ramifications into the primary somatosensory cortex (S1). The sensory networks in panels $\mathbf{A}, \mathbf{B}, \mathbf{D}, \mathbf{E}$ are based on textbook descriptions (e.g., Watson et al., 2011) and include the relevant structures given by the ABA. The sensory pathways can be described using the ABA. However, the ABA needs refinements to distinguish lower and upper limb (especially hind and forelimb), see panel E. The ABA does not include a division of the barrel field of the primary somatosensory areas, see panels $\mathbf{C}-\mathbf{D}$. The networks in panels $\mathbf{A}, \mathbf{B}, \mathbf{D}, \mathbf{E}$ indicate information flows to areas that do not necessarily terminate in the primary sensory areas of the isocortex, such as the hypothalamic and midbrain targets of the retinal ganglion cells in panel $\mathbf{A}$, and the midbrain nuclei related to whisker movements in panel D. These connections are well known and usually not discussed regarding sensory processing in textbooks. As a result, visual and whisker system meet in the SuCo regarding eye and whisker movements. Note that the model is agnostic about the (sensory) information, meaning that nuclei may be activated but the object of processing (information) is less defined. Meaning is attached by the vast amount of studies about the physiology of the nuclei. For instance, the hypothalamic nuclei play a role in the circadian timing system, and the nuclei in the midbrain (OT, PPT, OPT) receive input from the retina (see panel A) and are known to be involved in eye movement coordination and reflexes. The ABA includes the following areas involved in sensory processing. The nuclei in the medulla are DC: dorsal cochlear nucleus, $\mathrm{CN}$ : cuneate nucleus, GN: gracile nucleus, 
1737 SpVc: caudal part of the spinal nucleus of the trigeminal, SpVi: interpolar part of the spinal 1738 nucleus of the trigeminal, SpVo: oral part of the spinal nucleus of the trigeminal, Tz: nucleus 1739 of the trapezoid body, and VC: ventral cochlear nucleus. The hypothalamic nuclei are AH: 1740 anterior hypothalamic nucleus, SCN: suprachiasmatic nucleus, and SPVZ: subparaventricular 1741 zone. Involved structures in the pons are NLL: nucleus of the lateral lemniscus, and PrV: 1742 principal sensory nucleus of the trigeminal. The sensory related nuclei of the midbrain are 1743 BIC: nucleus of the brachium of the inferior colliculus, IC: inferior colliculus, MeV: 1744 trigeminal nucleus, OT: nucleus of the optic tract, OPT: olivary pretectal nucleus, PPT: 1745 posterior pretectal nucleus, SuCo: superior colliculus, sensory related. The thalamic nuclei 1746 involved in the sensory pathways are dLGN: dorsal part of the lateral geniculate complex, 1747 IGL: intergeniculate leaflet of the lateral geniculate complex, LD: lateral dorsal nucleus, LPN: 1748 lateral posterior nucleus, MG: medial geniculate complex, Po: posterior complex, vLGN: 1749 ventral part of the lateral geniculate complex, VPL: ventral posterolateral nucleus, and VPM: 1750 ventral posteromedial nucleus. The sensory brain areas in the isocortex are A1: primary 1751 auditory area, V1: Primary visual area, V2: secondary visual area, S1 primary somatosensory cortex, S2: secondary somatosensory cortex, and M1: primary motor cortex. Note that the brain areas are listed in Table 2 and Table 3. 
a

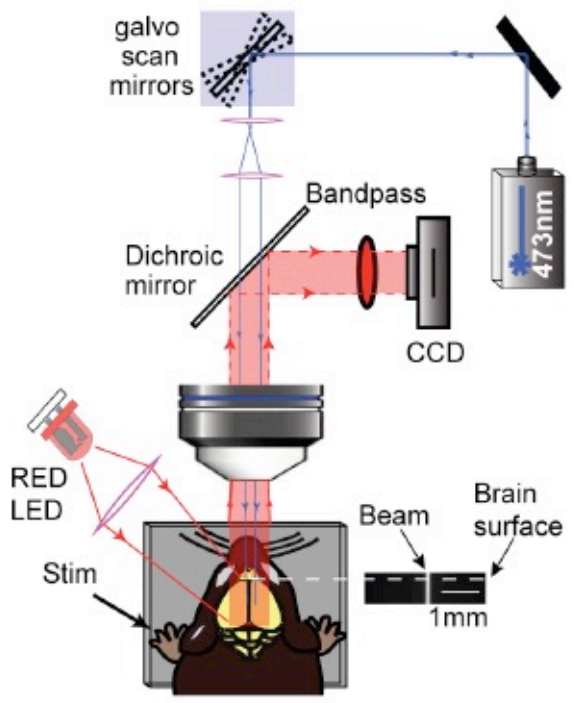

C

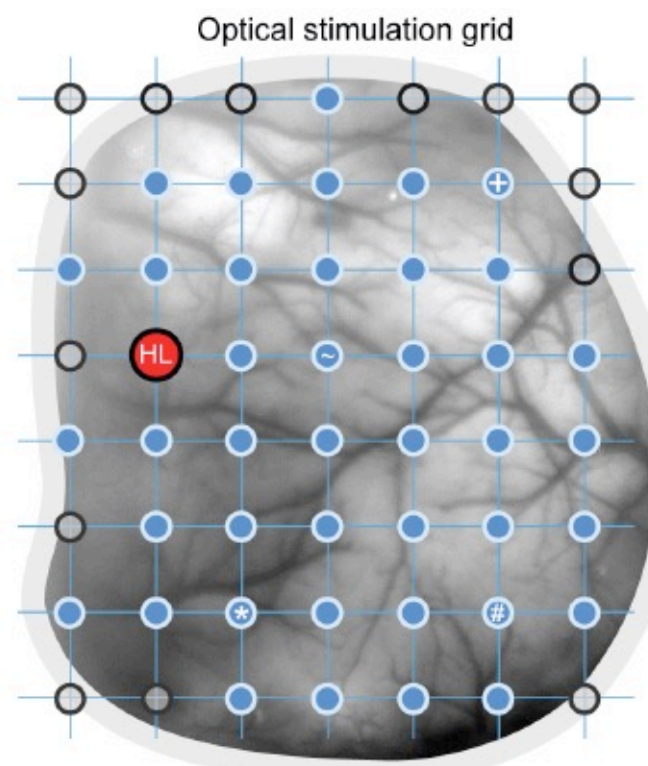

b

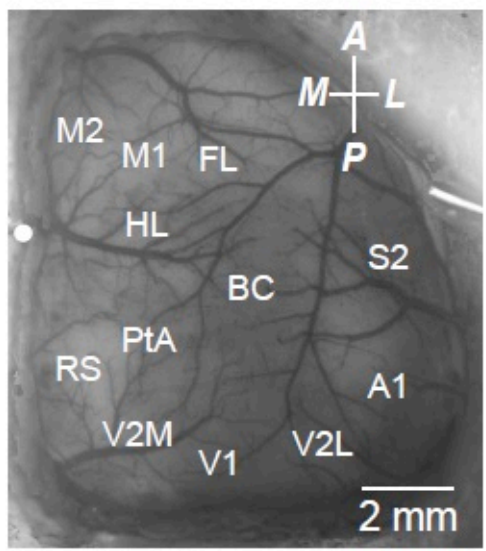

1754 Figure 7 Experimental apparatus and method to investigate brain response to stimulation.

1755 Panel A: The schematic of the experimental set-up. This set-up consists of a $473 \mathrm{~nm}$ laser 1756 generator whose beam is directed by galvo scan mirrors to a specific point on the cortical 1757 surface. By changing the configuration of the galvo scan mirrors, we can change the 1758 coordinates of the stimulated point. The CCD camera captures the voltage activity of the 1759 brain. Panel B: An example of a cranial window over the mice cortex prepared for VSD 1760 imaging. The cortical regions are labeled based on their coordinates with respect to the 1761 bregma, marked by white circle. Panel C: An example of the grid points mapped on the 1762 cortical surface by galvo scans mirrors. The laser can stimulate each one of these points. 1763 Examples of the voltage activity right after stimulation of some of these points are presented 1764 on right. 

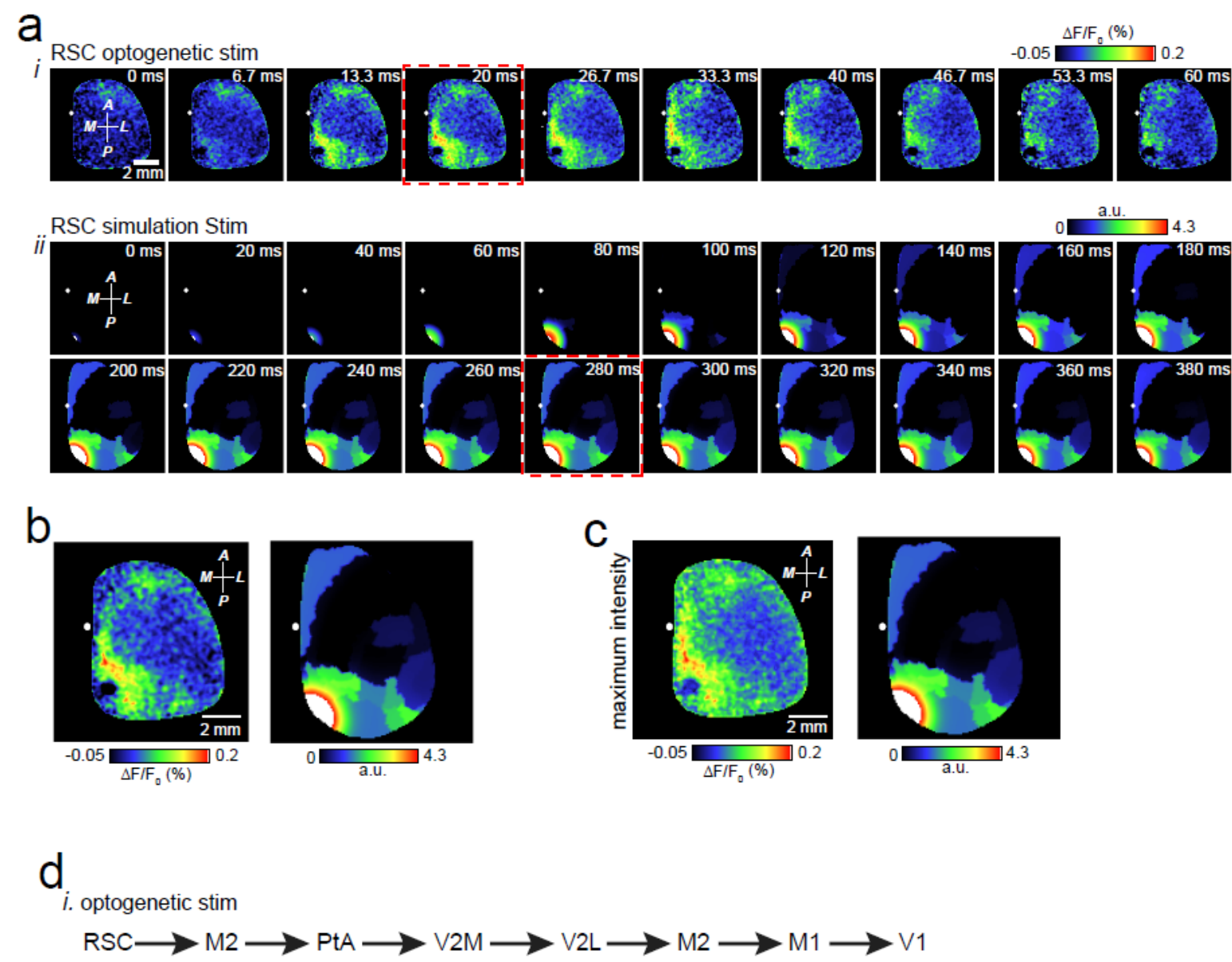

ii. simulation stim

$\mathrm{RSC} \longrightarrow \mathrm{V} 2 \mathrm{M} \longrightarrow \mathrm{PtA} \longrightarrow \mathrm{V} 2 \mathrm{~L} \longrightarrow \mathrm{V} 1 \longrightarrow \mathrm{HL} \longrightarrow \mathrm{M} 2 \longrightarrow \mathrm{S}_{2}$ nose

1765 Figure 8 Brain response after stimulation. Panel A: The spatiotemporal pattern of voltage

1766 activity after (i) optogenetic and (ii) simulated stimulation of RSC. Panel B: The enlarged

1767 frames, highlighted by a red rectangle in Panel A, juxtaposed for easier comparison. Panel C:

1768 The spatial distribution of the post-stimulus maximum voltage activity Panel $\mathbf{D}$ : The temporal

1769 order of activation of cortical regions after optogenetic (i) and simulated (ii) stimulation.

1770 Activation occurs when the voltage level of a given region surpasses $20 \%$ of its peak activity

1771 after stimulation was applied. 

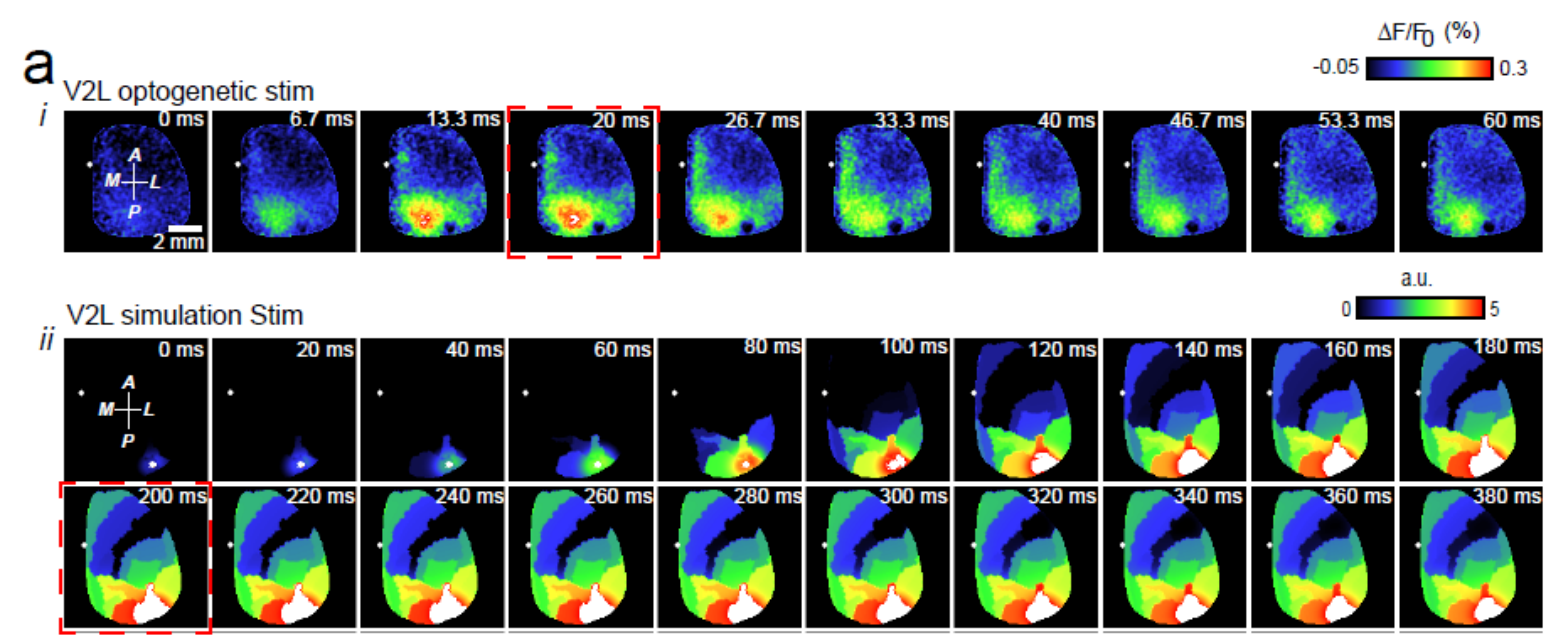

\section{b}
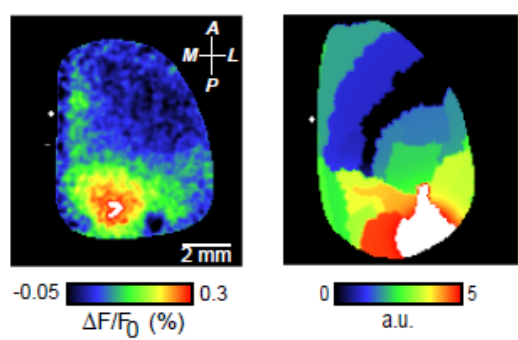

d

optogenetic stim

$\mathrm{V} 2 \mathrm{~L} \longrightarrow \mathrm{PtA} \longrightarrow \mathrm{V} 2 \mathrm{M} \longrightarrow \mathrm{V} 1 \rightarrow \mathrm{M} 2 \rightarrow \mathrm{RSC} \longrightarrow \mathrm{BC}$

simulation stim

$\mathrm{V} 2 \mathrm{~L} \longrightarrow \mathrm{V} 1 \longrightarrow \mathrm{A} 1 \longrightarrow \mathrm{PtA} \longrightarrow \mathrm{V} 2 \mathrm{M} \longrightarrow \mathrm{RSC} \longrightarrow \mathrm{BC}$

1772 Figure 9 Panel A: The spatiotemporal pattern of voltage activity after optogenetic (i) and

1773 simulated (ii) stimulation of V2L. Panel B: The enlarged frames, specified by a red rectangle

1774 in Panel A, juxtaposed for easier comparison. Panel C: The spatial distribution of the post-

1775 stimulus maximum voltage activity. Panel D: The temporal order of activation of cortical

1776 regions after optogenetic. (i) and simulated (ii) stimulation. Activation occurs when the

1777 voltage level of a given region surpasses $20 \%$ of its peak activity after stimulation was

1778 applied. 


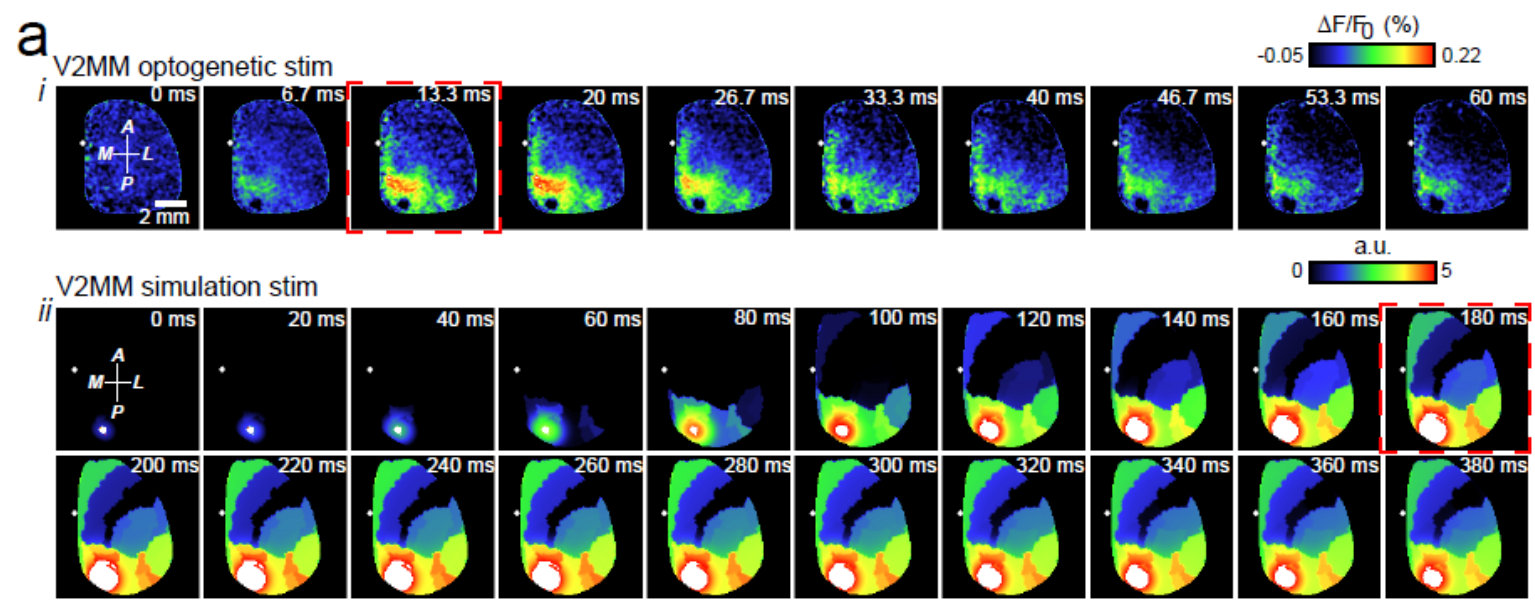

b
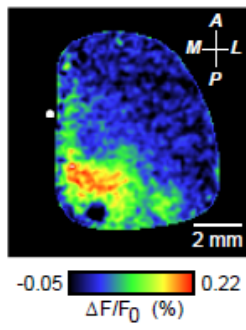

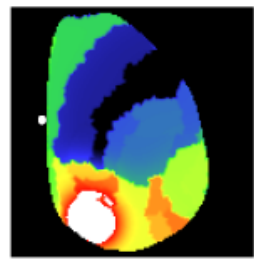

a.u. 5
C

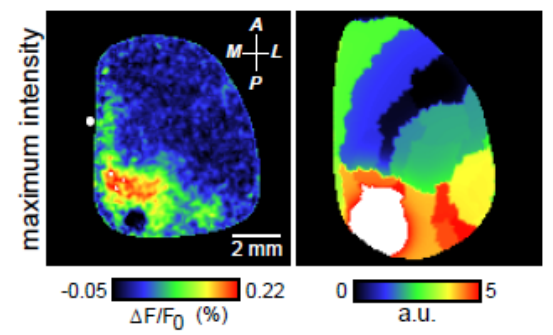

d

optogenetic stim

$\mathrm{V} 2 \mathrm{M} \longrightarrow \mathrm{M} 2 \longrightarrow \mathrm{RSC} \longrightarrow \mathrm{PtA} \longrightarrow \mathrm{V} 1 \longrightarrow \mathrm{V} 2 \mathrm{~L}$

simulation stim

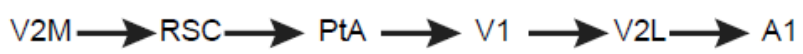

1779 Figure 10 Panel A: The spatiotemporal pattern of voltage activity after optogenetic (i) and

1780 simulated (ii) stimulation of V2M. Panel B: The enlarged frames, specified by a red rectangle

1781 in Panel A, juxtaposed for easier comparison. Panel C: The spatial distribution of the post-

1782 stimulus maximum voltage activity. Panel D: The temporal order of activation of cortical

1783 regions after optogenetic (i) and simulated (ii) stimulation. Activation occurs when the

1784 voltage level of a given region surpasses $20 \%$ of its peak activity after stimulation was

1785 applied.

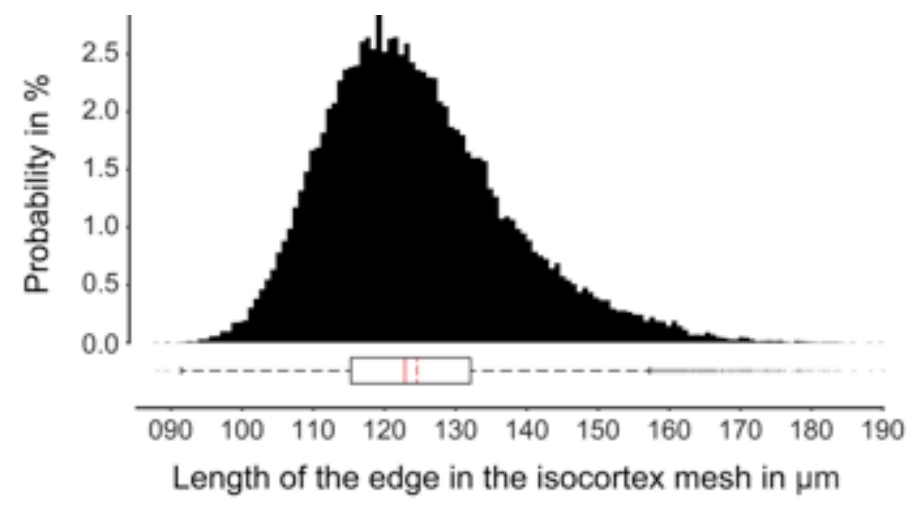


1786 Figure 11. The sharp unimodal distribution of edge lengths indicates the quality of the 1787 reconstructed surface of the isocortex using a regular-triangle mesh of 27,554 triangles with

178841,524 edges. The expectation and the standard deviation is $(124.5786 \pm 13.0144) \mu \mathrm{m}$. The 1789 edge lengths are positively skewed (0.7164) due to positive definite length (minimum and 1790 maximum lengths are $88.1525 \mu \mathrm{m}$ and $190.0629 \mu \mathrm{m})$. 


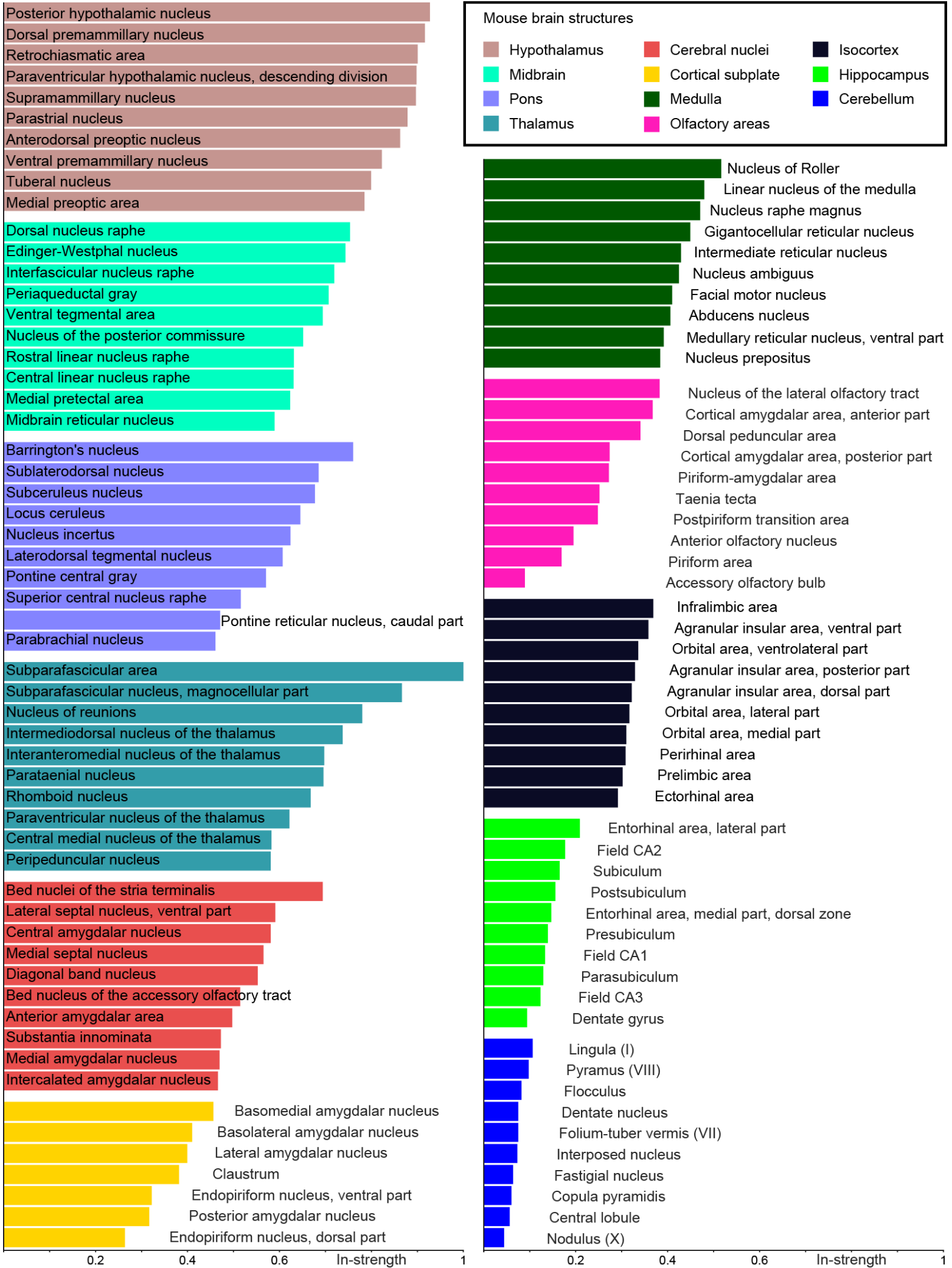

1791 Figure 12. In-strength indicates the income of a brain area. The hypothalamus receives the 1792 strongest input in the mouse brain model and the cerebellum the weakest. The isocortex is 1793 listed 9 out of 11 structures. The structures are ordered by their mean in-strength. This 1794 indicates that the subcortical structures are highly interdependent and the isocortex might be 1795 sensitive to subcortical input but autonomous as well. For each structure (e.g., thalamus) 10 
areas are listed in order of the highest in-strength. Note that the cortical subplate is divided in seven areas.

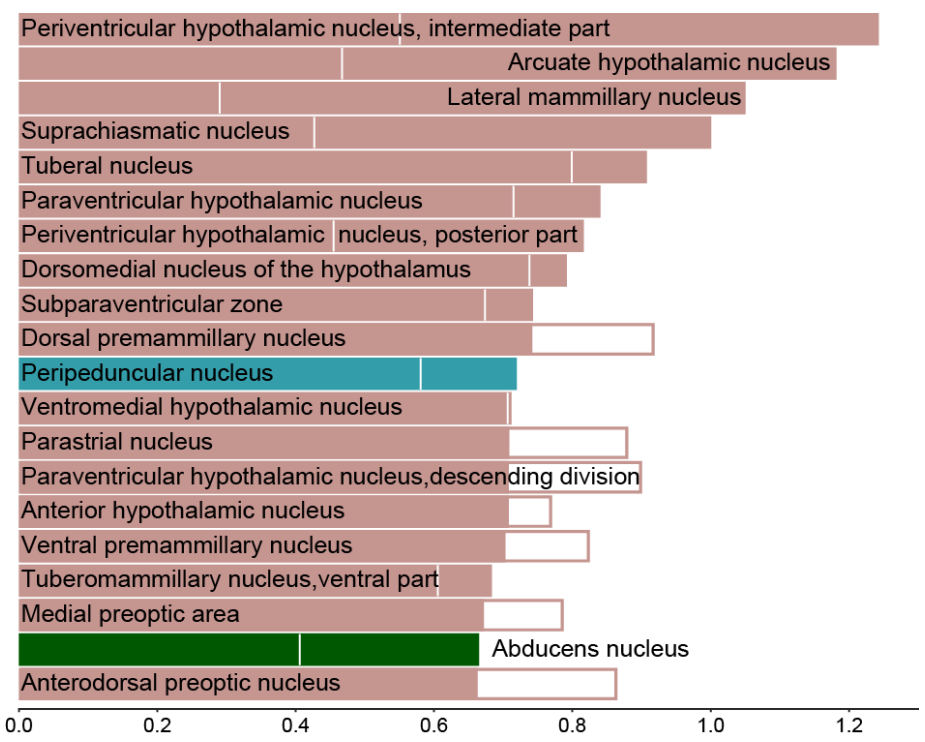

1798 Figure 13. Out-strength indicates that areas in the hypothalamus project the most in the mouse brain model. The color-code for the structures is the same as in Figure 12: brown indicates areas in the hypothalamus, dark cyan the thalamus, and dark green medulla. The white vertical lines in a bar and the white filled bars indicate the in-strength of an area.

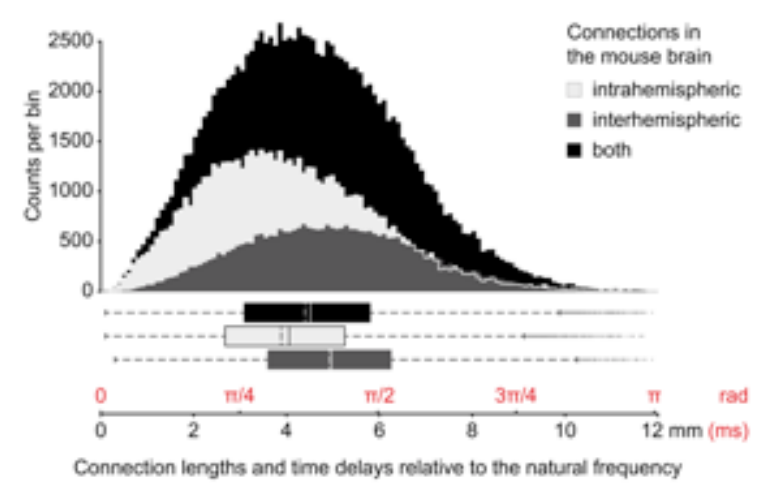

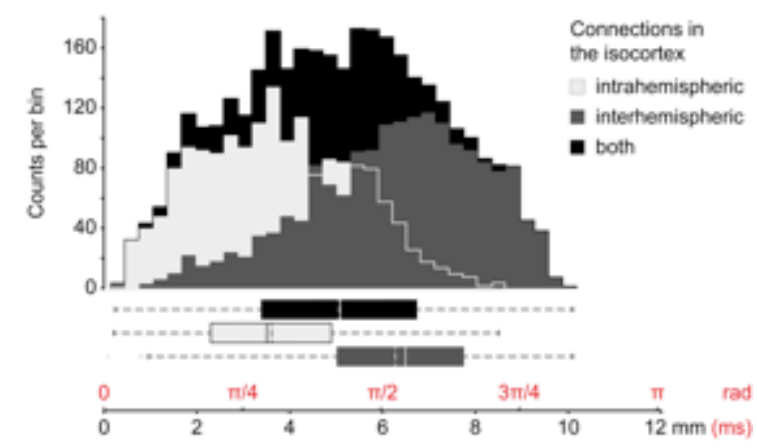

Connection lengths and time delays relative to the natural frequency

1802 Figure 14. Histograms of the distances between brain areas from the ABA used to

1803 approximate the tract lengths of long-range connections. Panel A: The distribution of 1804 connections in the entire mouse brain is slightly skewed but unimodal with a mean and 1805 median of about $4.511 \mathrm{~mm}$ and $4.394 \mathrm{~mm}$. The time delays due to the signal transmission 1806 shift the local activity by half a period via the longest connections and mostly by about $3 \pi / 8$ 1807 for medium-length connections. The distances directly translate into time delays by assuming 1808 an average conduction speed for white matter axonal fibers of $1 \mathrm{~m} / \mathrm{s}$. The connections appear 1809 of similar length regarding the entire mouse in panel $\mathbf{A}$, the connections differ regarding the 1810 isocortex in panel B. The intrahemispheric connections are shorter than the connections 1811 between the isocortical hemispheres. This result confirms the report in Braitenberg and Schüz, 
1812

1813

1814

1815

1816

1817

1818

1819

1820

1821

1822

1823

1824

1825

1826

1827

1828

1829

1830

1831

1832

1833

1834

1835

1836

1837

1838

1839

1998, Chapter 26, of a second peak in the histogram whose significance is obscured. The isocortical connections are outnumbered, thus their quantitative effect on the entire mouse, in A, is marginal. Time delays translate into shifts of transmitted local brain activity. The local activity in the network model of the mouse brain is assumed to primarily convey the natural frequency at each brain area (about $42 \mathrm{~Hz}$ ). The time delays translate into a phase shift in local activity throughout its transmission. The number of bins is 119 with a bin width of $0.0889 \mathrm{~mm}$ in panel $\mathbf{A}$ and 17 bins of $0.3056 \mathrm{~mm}$ width in panel $\mathbf{B}$. The number of bins in the histogram, $n_{\text {bins, }}$, was calculated according to $n_{\text {bins }}=\exp (0.626+0.4 \log (n-1))$, with the number of connections $n$ (Otnes and Enochson, 1972), which is listed in Table 4. The statistics are given in Table 5.
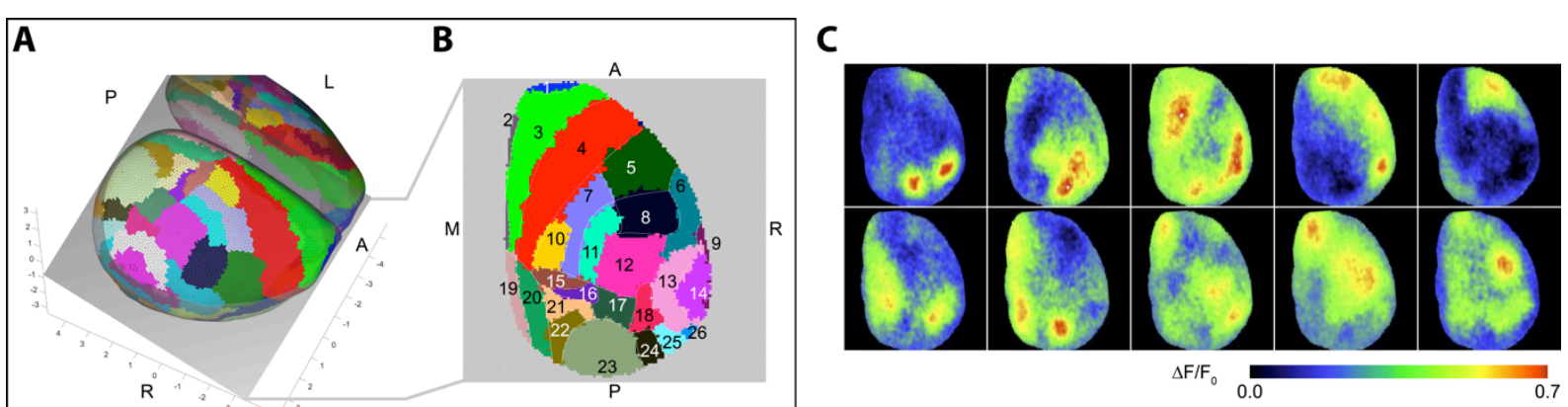

Figure 15. Modeling the focal plane for voltage sensitive dye (VSD) imaging. Panel A: The camera setup in the mice experiments was reproduced in the 3 dimensional geometric model of the mouse brain. The focus was first upon the surface of the right isocortex and then adjusted $1 \mathrm{~mm}$ inside the cortex so that the depth of field covers most of the right isocortex (for the experimental setup see Mohajerani et al., 2013). The consequence is that the focal plane cuts through the right isocortex (see the gray plane in A) and records brain activity from a wide field of the isocortex. Panel B: The visible neural masses are projected onto the focal plane, using the normal vector of the focal plane (i.e., assuming an infinite focal length). The modeled focal plane is sampled by an array of $128 \times 128$ pixels in accordance with the camera sensor used in the experiments for the VSD imaging recordings. Because, each brain area in the isocortex comprises several neural masses, the areas are colored in both panels $\mathbf{A}$ and $\mathbf{B}$. The areas captured by the VSD imaging are numbered in panel $\mathbf{B}$ and listed accordingly in Table 6. The labels A, P, L, R, M denote locations: (A)nterior, (P)osterior, (L)eft, (R)ight, and (M)edial. Panel C: Experimental VSD imaging of spontaneous brain activity in isoflurane anesthetized mouse show spatial organization (here ten exemplary patterns) demonstrating the qualitative correspondence of experimental and modeled VSD imaging purely based on the ABA parcellation. Note that the (secondary and primary) motor areas (areas 3 in green and 4 in red in the panels $\mathbf{A}$ and $\mathbf{B}$ ) and such somatosensory areas as 
1840 the barrel field (area 12 in magenta in the panels $\mathbf{A}$ and $\mathbf{B}$ ) represent spatially extended areas

1841 that are functionally undivided (e.g., by topographic maps) in the ABA. The VSD imaging

1842 can indicate activity patterns that are finer grained than the used ABA division of the

1843 isocortex into brain areas in the model. The snapshots of the experimental VSD imaging of

1844 spontaneous activity in panel $\mathbf{C}$ are taken and adapted with permission from already published

1845 data (Supplementary Video 2) by Mohajerani et al., 2013. 


\section{TABLES}

1846 Table 1. Mapping of brain areas in the mouse model, that is, areas to brain structures

1847 following the division of the Allen Brain Atlas (ABA; http://connectivity.brain-map.org).

1848 Because of a total of 512 brain areas in the model, only the number of areas is listed. The 1849 names are listed in Table 2 and Table 3.

\begin{tabular}{|c|c|c|}
\hline Structure & Division & Areas \\
\hline \multirow[t]{4}{*}{ Cortex } & Isocortex & 42 \\
\hline & Olfactory areas & 11 \\
\hline & Hippocampal formation & 10 \\
\hline & Cortical subplate & 7 \\
\hline \multirow[t]{2}{*}{ Cerebral nuclei } & Striatum & 13 \\
\hline & Pallidum & 8 \\
\hline \multirow[t]{2}{*}{ Thalamus } & Sensory-motor-related & 13 \\
\hline & Polymodal association cortex related & 8 \\
\hline \multirow[t]{4}{*}{ Hypothalamus } & Periventricular zone & 3 \\
\hline & Periventricular region & 11 \\
\hline & Medial zone & 12 \\
\hline & Lateral zone & 7 \\
\hline \multirow[t]{3}{*}{ Midbrain } & Sensory related & 6 \\
\hline & Motor related & 17 \\
\hline & Behavior-state related & 7 \\
\hline \multirow[t]{3}{*}{ Pons } & Sensory related & 3 \\
\hline & Motor related & 7 \\
\hline & Behavior-state related & 7 \\
\hline \multirow[t]{2}{*}{ Medulla } & Sensory related & 9 \\
\hline & Motor related & 23 \\
\hline \multirow[t]{2}{*}{ Cerebellum } & Cortex & 12 \\
\hline & Nuclei & 3 \\
\hline
\end{tabular}

1850 Table 2. Cerebral brain structures and their division. Number of nodes per area in brackets

1851 (left, right).

Isocortex (42 areas):

Frontal pole, cerebral cortex $(128,165)$

Primary motor area $(667,605)$

Secondary motor area $(489,483)$

Primary somatosensory area, nose $(167,185)$

Primary somatosensory area, barrel field $(212,215)$

Primary somatosensory area, lower limb $(89,85)$
Rostrolateral visual area $(69,71)$

Temporal association areas $(145,148)$

Perirhinal area $(188,228)$

Ectorhinal area $(151,163)$

.


Primary somatosensory area, mouth $(269,265) \quad$ Main olfactory bulb $(1,1)$

Primary somatosensory area, upper $\operatorname{limb}(148,153)$ Accessory olfactory bulb $(1,1)$

Primary somatosensory area, trunk $(58,53)$

Anterior olfactory nucleus $(1,1)$

Primary somatosensory area, unassigned $(88,100)$

Taenia tecta $(1,1)$

Supplemental somatosensory area $(271,223)$

Dorsal peduncular area $(1,1)$

Gustatory areas $(144,141)$

Visceral area $(94,120)$

Piriform area $(1,1)$

Dorsal auditory area $(147,173)$

Nucleus of the lateral olfactory tract $(1,1)$

Cortical amygdalar area, anterior part $(1,1)$

Primary auditory area $(125,113)$

Cortical amygdalar area, posterior part $(1,1)$

Ventral auditory area $(112,138)$

Piriform-amygdalar area $(1,1)$

Anterolateral visual area $(58,63)$

Postpiriform transition area $(1,1)$

Anteromedial visual area $(52,59)$

Lateral visual area $(56,61)$

Primary visual area $(415,404)$

Posterolateral visual area $(126,117)$

Posteromedial visual area $(92,78)$

Laterointermediate area $(47,46)$

Postrhinal area $(130,134)$

Anterior cingulate area, dorsal part $(224,246)$

Anterior cingulate area, ventral part $(147,138)$

Prelimbic area $(105,98)$

Infralimbic area $(106,117)$

Orbital area, lateral part $(108,96)$

Orbital area, medial part $(90,88)$

Orbital area, ventrolateral part $(125,100)$

Agranular insular area, dorsal part $(169,173)$

Agranular insular area, posterior part $(151,147)$

Agranular insular area, ventral part $(140,138)$

Hippocampal Formation (10 areas):

Field CA1

Field CA2

Field CA3

Dentate gyrus

Entorhinal area, lateral part

Entorhinal area, medial part, dorsal zone

Parasubiculum

Postsubiculum

Presubiculum

Subiculum

Cortical Subplate ( 7 areas):

Claustrum

Endopiriform nucleus, dorsal part

Endopiriform nucleus, ventral part

Retrosplenial area, lateral agranular part $(132,162)$ Lateral amygdalar nucleus

Retrosplenial area, dorsal part $(329,251)$

Basolateral amygdalar nucleus

Retrosplenial area, ventral part $(410,377)$

Basomedial amygdalar nucleus

Anterior area $(47,32)$

Posterior amygdalar nucleus

1852 Table 3. Subcortical brain structures and their division. One network node per area.

Cerebral Nuclei (21 areas):

Caudoputamen

Nucleus accumbens

Fundus of striatum

Olfactory tubercle

Lateral septal nucleus, caudal (caudodorsal) part

Lateral septal nucleus, rostral (rostroventral) part
Anteroventral nucleus

Anteromedial nucleus

Anterodorsal nucleus

Interanteromedial nucleus

Interanterodorsal nucleus

Lateral dorsal nucleus

Intermediodorsal nucleus 
Lateral septal nucleus, ventral part

Septofimbrial nucleus

Anterior amygdalar area

Bed nucleus of the accessory olfactory tract

Central amygdalar nucleus

Intercalated amygdalar nucleus

Medial amygdalar nucleus

Globus pallidus, external segment

Globus pallidus, internal segment

Substantia innominata

Magnocellular nucleus

Medial septal nucleus

Diagonal band nucleus

Triangular nucleus of septum

Bed nuclei of the stria terminalis

Thalamus (38 areas):

Ventral anterior-lateral complex

Ventral medial nucleus

Ventral posterolateral nucleus

Ventral posteromedial nucleus

Ventral posteromedial nucleus, parvicellular part

Subparafascicular nucleus, magnocellular part

Subparafascicular nucleus, parvicellular part

Subparafascicular area

Peripeduncular nucleus

Medial geniculate complex

Dorsal part of the lateral geniculate complex

Lateral posterior nucleus

Posterior complex

Posterior limiting nucleus

Suprageniculate nucleus
Mediodorsal nucleus

Submedial nucleus

Perireunensis nucleus

Paraventricular nucleus

Parataenial nucleus

Nucleus of reunions

Rhomboid nucleus

Central medial nucleus

Paracentral nucleus

Central lateral nucleus

Parafascicular nucleus

Reticular nucleus

Intergeniculate leaflet of the lateral geniculate group

Ventral part of the lateral geniculate complex

Medial habenula

Hypothalamus (33 areas):

Paraventricular nucleus

Periventricular nucleus, intermediate part

Arcuate nucleus

Anterodorsal preoptic nucleus

Anteroventral preoptic nucleus

Anteroventral periventricular nucleus

Dorsomedial nucleus

Median preoptic nucleus

Medial preoptic area

Parastrial nucleus

Periventricular nucleus, posterior part

Periventricular nucleus, preoptic part

Subparaventricular zone

Suprachiasmatic nucleus

Anterior nucleus

Table is continued next page. 
1854 Subcortical brain structures and their division (continued).

Hypothalamus (continued)

Lateral mammillary nucleus

Medial mammillary nucleus

Supramammillary nucleus

Tuberomammillary nucleus, dorsal part

Tuberomammillary nucleus, ventral part

Medial preoptic nucleus

Dorsal premammillary nucleus

Ventral premammillary nucleus

Paraventricular nucleus, descending division

Ventromedial nucleus

Posterior nucleus

Lateral area

Lateral preoptic area

Parasubthalamic nucleus

Retrochiasmatic area

Subthalamic nucleus

Tuberal nucleus

Zona incerta

Midbrain (30 areas)

Superior colliculus, sensory related

Inferior colliculus

Nucleus of the brachium, inferior colliculus

Nucleus sagulum

Parabigeminal nucleus

Midbrain trigeminal nucleus

Substantia nigra, reticular part

Ventral tegmental area

Midbrain reticular nucleus, retrorubral area

Midbrain reticular nucleus

Superior colliculus, motor related

Periaqueductal gray

Anterior pretectal nucleus

Medial pretectal area

Nucleus of the optic tract

Nucleus of the posterior commissure

Olivary pretectal nucleus

Posterior pretectal nucleus
Cuneiform nucleus

Red nucleus

Oculomotor nucleus

Edinger-Westphal nucleus

Ventral tegmental nucleus

Substantia nigra, compact part

Pedunculopontine nucleus

Interfascicular nucleus raphe

Interpeduncular nucleus

Rostral linear nucleus raphe

Central linear nucleus raphe

Dorsal nucleus raphe

Pons (17 areas)

Nucleus of the lateral lemniscus

Principal sensory nucleus of the trigeminal

Parabrachial nucleus

Barrington's nucleus

Pontine central gray

Pontine gray

Pontine reticular nucleus, caudal part

Supratrigeminal nucleus

Tegmental reticular nucleus

Motor nucleus of trigeminal

Superior central nucleus raphe

Locus ceruleus

Laterodorsal tegmental nucleus

Nucleus incertus

Pontine reticular nucleus

Subceruleus nucleus

Sublaterodorsal nucleus

Medulla (32 areas)

Dorsal cochlear nucleus

Ventral cochlear nucleus

Cuneate nucleus

Gracile nucleus

Nucleus of the trapezoid body

Nucleus of the solitary tract 
1855 Subcortical brain structures and their division (continued).

\begin{tabular}{|c|c|}
\hline Medulla (continued) & Hypoglossal nucleus \\
\hline Spinal nucleus of the trigeminal, caudal part & Nucleus raphe magnus \\
\hline Spinal nucleus of the trigeminal, interpolar part & Superior vestibular nucleus \\
\hline Spinal nucleus of the trigeminal, oral part & Nucleus $\mathrm{x}$ \\
\hline Abducens nucleus & Spinal vestibular nucleus \\
\hline \multicolumn{2}{|l|}{ Facial motor nucleus } \\
\hline Nucleus ambiguus & Cerebellum (15 areas) \\
\hline Gigantocellular reticular nucleus & Lingula (I) \\
\hline Inferior olivary complex & Central lobule \\
\hline Intermediate reticular nucleus & Culmen \\
\hline Linear nucleus of the medulla & Folium-tuber vermis (VII) \\
\hline Lateral reticular nucleus & Pyramus (VIII) \\
\hline Magnocellular reticular nucleus & Nodulus (X) \\
\hline Medullary reticular nucleus, dorsal part & Simple lobule \\
\hline Medullary reticular nucleus, ventral part & Ansiform lobule \\
\hline Parvicellular reticular nucleus & Paramedian lobule \\
\hline Paragigantocellular reticular nucleus, dorsal part & Copula pyramidis \\
\hline Paragigantocellular reticular nucleus, lateral part & Paraflocculus \\
\hline Nucleus of Roller & Flocculus \\
\hline Nucleus prepositus & Fastigial nucleus \\
\hline Lateral vestibular nucleus & Interposed nucleus \\
\hline Medial vestibular nucleus & Dentate nucleus \\
\hline
\end{tabular}

1856 Table 4. The number of connections among areas in the isocortex is outnumbered by other 1857 areas in the mouse brain model. The table lists the number of connections of the mouse brain 1858 model and its division into isocortex and sub-isocortex. The number of different areas is given 1859 in brackets.

\begin{tabular}{rlcr}
\hline & \multicolumn{3}{c}{ Structure } \\
\cline { 2 - 4 } Area & Mouse brain (512) & Isocortex (84) & Sub-Isocortex (428) \\
\hline Intra & 13,0560 & 003,444 & 091,592 \\
Inter & 13,1072 & 003,528 & 091,164 \\
All & 26,1632 & 006,972 & 182,756 \\
\hline
\end{tabular}

1860 Table 5. Statistics of connection lengths in the mouse brain model and its subdivision. Note 1861 the difference between intra- and inter-iscocortical connections in terms of expectation, 
1862 median (i.e., 50\% quantile), and variance. All values are in $\mathrm{mm}$, except the variance that is in $1863 \mathrm{~mm}^{2}$ and the skewness that is a bare number.

\begin{tabular}{lcccccccc}
\hline Parameter & \multicolumn{2}{c}{ Mouse brain } & & \multicolumn{2}{c}{ Isocortex } & & \multicolumn{2}{c}{ Sub-isocortex } \\
\cline { 2 - 3 } & Intra & Inter & & Intra & Inter & & Intra & Inter \\
\hline Minimum & 0.1085 & 0.1083 & & 0.4441 & 0.2352 & & 0.1085 & 0.1083 \\
Maximum & 11.6731 & 11.8715 & 8.5598 & 10.0594 & & 11.6731 & 11.8715 \\
Expectation & 4.0562 & 4.9721 & 3.7661 & 6.3171 & 3.6806 & 4.4273 \\
Variance & 3.3677 & 3.4961 & 2.8349 & 3.5418 & 3.0860 & 2.9773 \\
Skewness & 0.5133 & 0.2307 & 0.2042 & -0.5012 & & 0.6632 & 0.3889 \\
Quantile-05\% & 1.3444 & 2.0139 & 1.1605 & 2.7377 & & 1.1909 & 1.7948 \\
Quantile-50\% & 3.8858 & 4.9148 & 3.6685 & 6.5294 & & 3.4521 & 4.3090 \\
Quantile-95\% & 7.2747 & 8.1711 & 6.5422 & 9.0240 & & 6.8353 & 7.3476 \\
Quantile-99\% & 9.0197 & 9.4866 & 7.7075 & 9.5383 & & 8.4382 & 8.7810 \\
\hline
\end{tabular}

1864 Table 6. The experimental voltage sensitive dye (VSD) imaging captures activity of 1865 isocortical brain areas that are given in the virtual mouse brain model by the ABA. The 1866 numbering (\#) of the 26 listed areas (out of 84 isocortical areas) in the isocortex (total number 1867 of 512 brain areas) captured by the VSD imaging technique corresponds to the labels in Panel 1868 B of Figure 15.

\begin{tabular}{cllll}
\hline$\#$ Areas captured by the VSD imaging & & $\#$ Areas captured by the VSD imaging \\
\hline 1 & Frontal pole & & 14 Primary auditory area \\
2 & Anterior cingulate area, dorsal part & & 15 Primary somatosensory area, trunk \\
3 & Secondary motor area & 16 Anterior area \\
4 & Primary motor area & 17 Rostrolateral visual area \\
5 & Primary somatosensory area, mouth & & 18 Anterolateral visual area \\
6 & Supplemental somatosensory area & & 19 Retrosplenial area, dorsal part \\
7 & Primary somatosensory area, upper limb & & 20 Retrosplenial area, lateral agranular part \\
8 & Primary somatosensory area, nose & & 21 Anteromedial visual area \\
9 & Ventral auditory area & & 22 Posteromedial visual area \\
10 & Primary somatosensory area, lower limb & & 23 Primary visual area \\
11 & Primary somatosensory area, unassigned & & 24 & Lateral visual area \\
12 & Primary somatosensory area, barrel field & & 25 Laterointermediate area \\
13 & Dorsal auditory area & 26 Temporal association areas \\
\hline
\end{tabular}




\section{SUPPLEMENTARY MATERIALS}

a
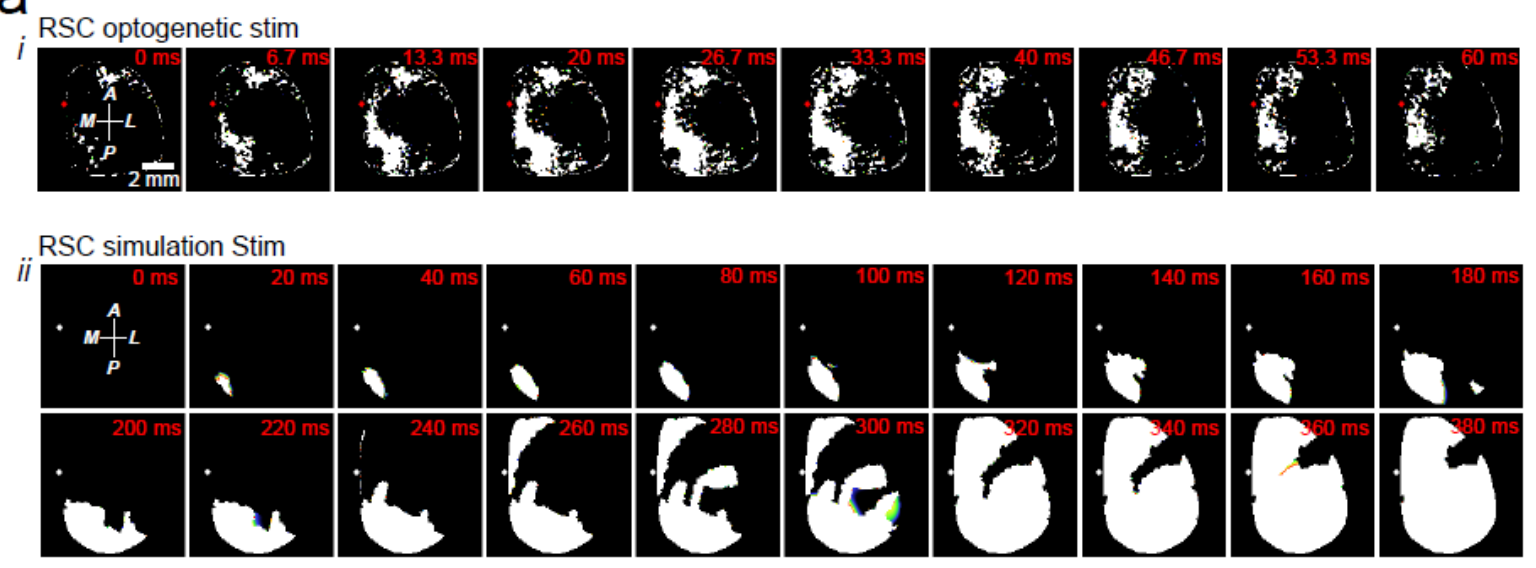

1869 Supplementary Figure 1 The spatiotemporal pattern of activation of cortical regions after

1870 optogenetic (i) and simulated (ii) stimulation of RSC. A region is defined as activated when

1871 its activity surpasses 20 percent of its peak activity after stimulation occurred. The white color

1872 represents the activated pixels at each frame (timestamp).

a
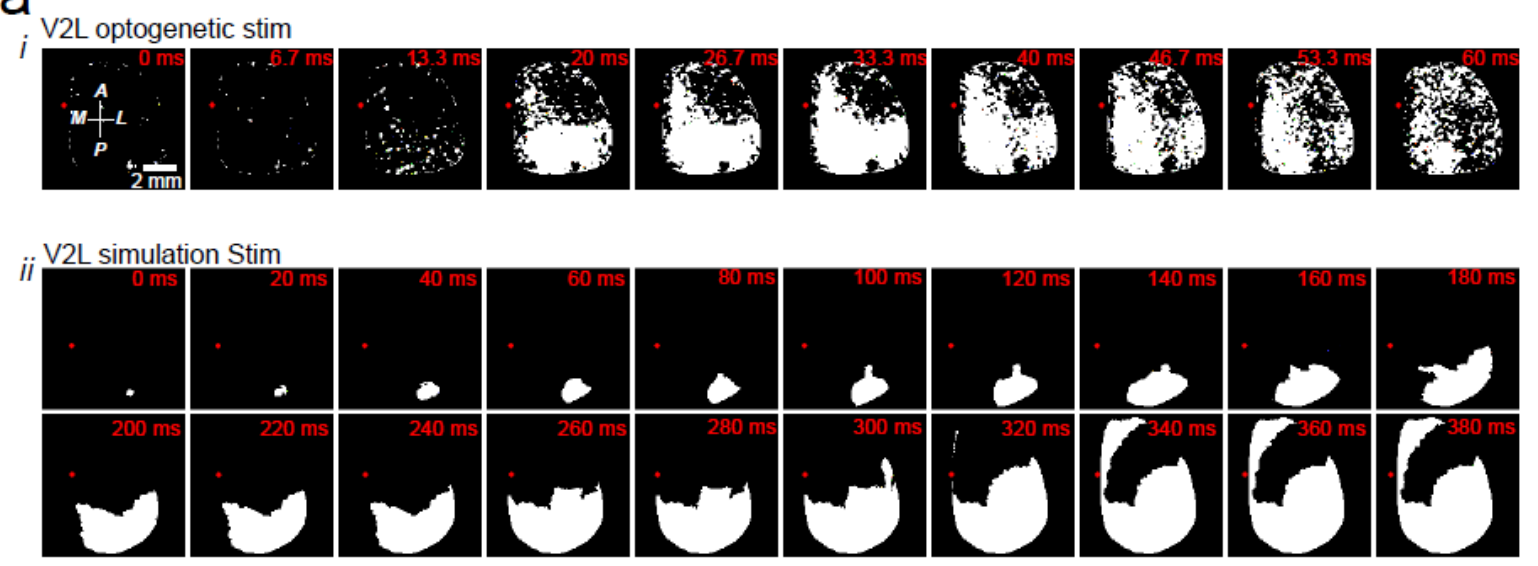

1873 Supplementary Figure 2 The spatiotemporal pattern of activation of cortical regions after

1874 optogenetic (i) and simulated (ii) stimulation of V2L. A region is defined as activated when

1875 its activity surpasses 20 percent of its peak activity after stimulation occurred. The white color

1876 represents the activated pixels at each frame (timestamp). 


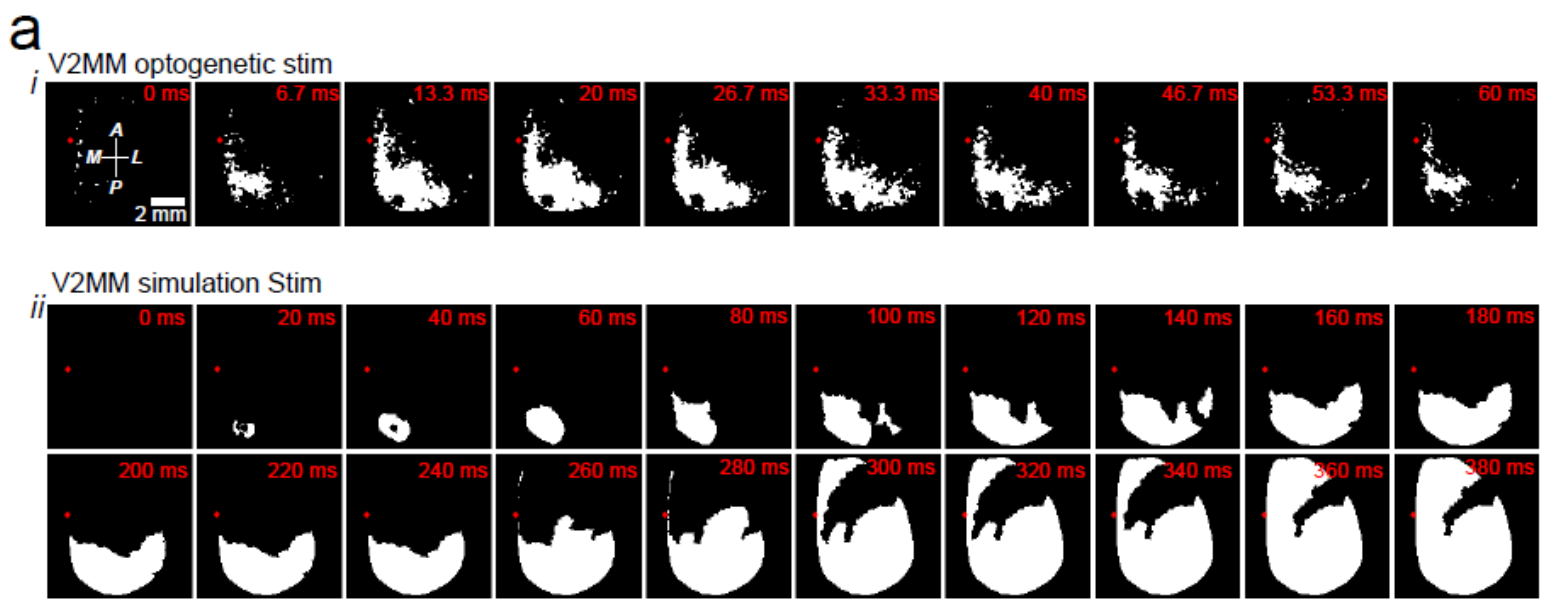

1877 Supplementary Figure 3 The spatiotemporal pattern of activation of cortical regions after

1878 optogenetic (i) and simulated (ii) stimulation of V2M. A region is defined as activated when

1879 its activity surpasses 20 percent of its peak activity after stimulation occurred. The white color

1880 represents the activated pixels at each frame (timestamp). 